\title{
Solid State Structures and Solution Behavior of Titanium(IV) Octahydrobinaphtholate Complexes. Examination of Nonlinear Behavior in the Asymmetric Addition of Ethyl Groups to Benzaldehyde
}

\author{
Karen M. Waltz, Patrick J. Carroll, and Patrick J. Walsh* \\ P. Roy and Diane T. Vagelos Laboratories, Department of Chemistry, University of \\ Pennsylvania, 231 South $34^{\text {th }}$ St., Philadelphia, Pennsylvania 19104-6323
}

Supporting Information

Table of Contents

Ortep of (meso)-6 with full atom labeling

Table S1. Data Collection Parameters for (meso)-6

Table S2. Atomic Coordinates for (meso)-6

Table S3. Thermal Parameters for (meso)-6

Table S4. Bond Distances for (meso)-6

Table S5. Bond Angles for (meso)-6

ORTEP of (meso)-7 with full atom labeling

Table S6. Data Collection Parameters for (meso)-7

Table S7. Atomic Coordinates for (meso)-7

Table S8. Thermal Parameters for (meso)-7

Table S9. Bond Distances for (meso)-7

Table S10. Bond Angles for (meso)-7

ORTEP of $\mathbf{8}$ with full atom labeling

Table S11. Data Collection Parameters for $\mathbf{8}$

Table S12. Atomic Coordinates for 8

Table S13. Thermal Parameters for 8

Table S14. Bond Distances for $\mathbf{8}$

Table S15. Bond Angles for 8

ORTEP of 9 with full atom labeling

Table S16. Data Collection Parameters for 9

Table S17. Atomic Coordinates for 9

Table S18. Thermal Parameters for 9

Table S19. Bond Distances for 9

Table S20. Bond Angles for 9

${ }^{1} \mathrm{H}$ and ${ }^{13} \mathrm{C}\left\{{ }^{1} \mathrm{H}\right\}$ NMR Spectra of (meso)-6, $(S, S)-6$, (meso)-7,

$\mathbf{8}$, and $\mathbf{9}$

${ }^{1} \mathrm{H}$ NMR Spectra of Titration Experiments of (meso)-6 and $(S, S)$-6 with 4,8 , and 14 equiv $\mathrm{Ti}(\mathrm{O}-i-\mathrm{Pr})_{4}$

${ }^{1} \mathrm{H}$ and ${ }^{13} \mathrm{C}\left\{{ }^{1} \mathrm{H}\right\}$ NMR Spectra of Reactions of $(R)$ - and ( rac $)$ $\mathrm{H}_{8}$-BINOL with 2 equiv $\mathrm{Ti}(\mathrm{O}-i \text { - } \mathrm{Pr})_{4}$

${ }^{1} \mathrm{H}$ and ${ }^{13} \mathrm{C}\left\{{ }^{1} \mathrm{H}\right\}$ NMR Spectra of Homochiral Dimer Formed

by Reaction of $(S)-\mathrm{H}_{8}$-BINOL with 1 equiv $\mathrm{Ti}(\mathrm{OCy})_{4}$

\begin{tabular}{|c|c|}
\hline No. of pages & page \\
\hline (1) & $\mathrm{S} 2$ \\
\hline (1) & S3 \\
\hline (2) & S4 \\
\hline (1) & S6 \\
\hline$(0.5)$ & S7 \\
\hline$(0.5)$ & S7 \\
\hline$(1)$ & S8 \\
\hline (1) & S9 \\
\hline (2) & S10 \\
\hline (1) & $\mathrm{S} 12$ \\
\hline$(0.5)$ & S13 \\
\hline$(0.5)$ & S13 \\
\hline$(1)$ & S14 \\
\hline (1) & S15 \\
\hline (3) & S16 \\
\hline (1) & S19 \\
\hline (1) & S20 \\
\hline (1) & S21 \\
\hline (1) & S22 \\
\hline (1) & S23 \\
\hline (5) & S24 \\
\hline (2) & S29 \\
\hline (1) & S31 \\
\hline (1.5) & S32 \\
\hline (10) & S34 \\
\hline (6) & S43 \\
\hline (4) & S49 \\
\hline (2) & S53 \\
\hline
\end{tabular}

Ortep of (meso)-6 with full atom labeling 


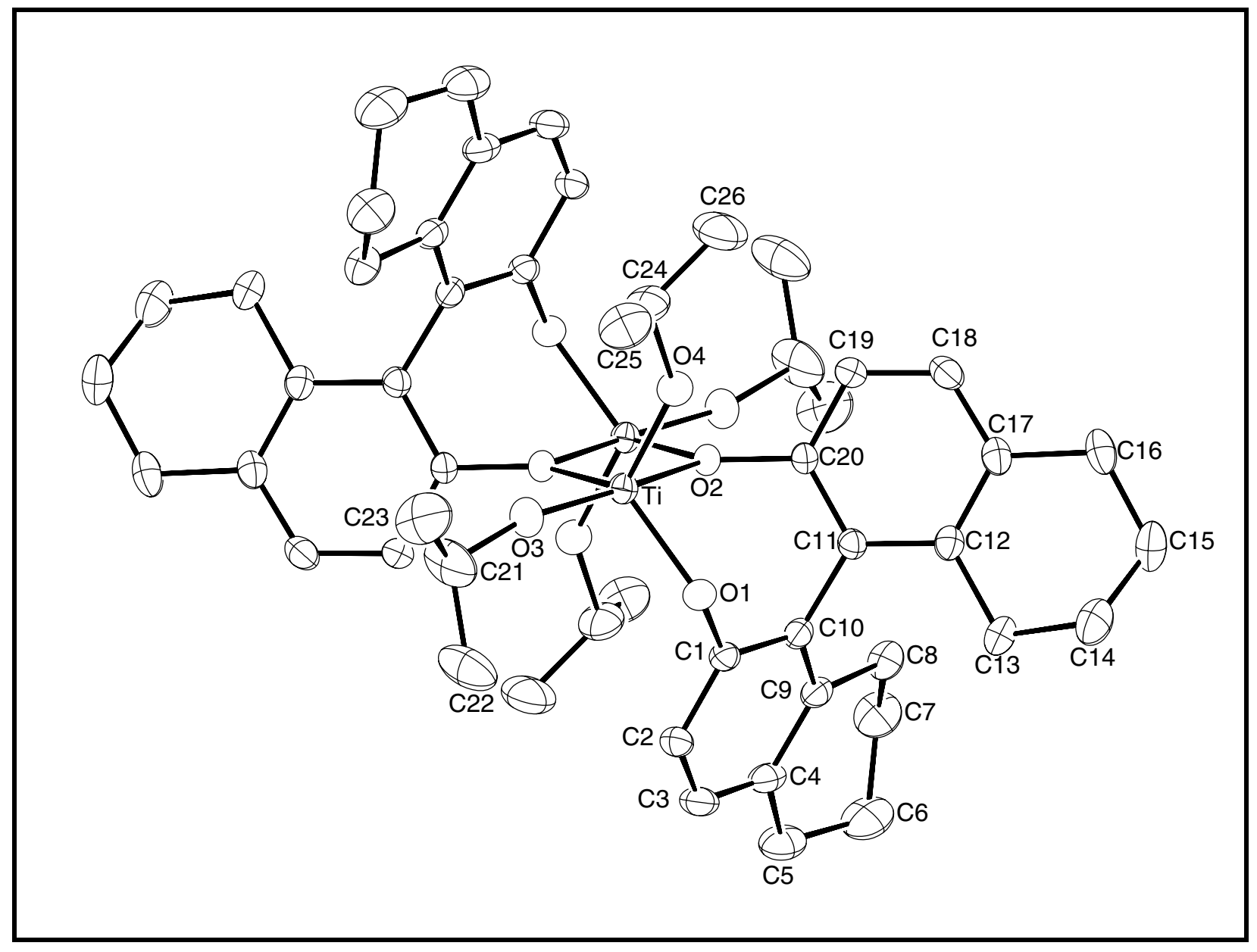


Table S1. Summary of Structure Determination of (meso)-6

Formula:

Formula weight:

Crystal class:

Space group:

$\mathrm{Z}$

Cell constants:

$\begin{array}{ll} & \mathrm{a} \\ & \mathrm{b} \\ & \mathrm{c} \\ & \beta \\ & \mathrm{V} \\ \mu & \\ \text { crystal size, } \mathrm{mm} \\ \mathrm{D}_{\text {calc }}\end{array}$

$\mathrm{F}(000)$

Radiation:

$2 \theta$ range

hkl collected:

No. reflections measured:

No. unique reflections:

No. observed reflections

No. reflections used in refinement

No. parameters

$\mathrm{R}$ indices $(\mathrm{F}>4 \sigma)$

$\mathrm{R}$ indices (all data)

GOF:

Final Difference Peaks, e/ $\AA^{3}$
$\mathrm{TiC}_{27} \mathrm{H}_{36} \mathrm{O}_{4} \mathrm{Cl}_{2}$

543.36

monoclinic

$\mathrm{P} 2_{1} / \mathrm{n}(\# 14)$

4

$12.7335(12) \AA$

$10.7575(12) \AA$

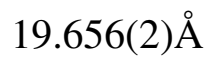

$93.633(7)^{\circ}$

2687.1(5) $\AA^{3}$

$5.48 \mathrm{~cm}^{-1}$

$0.30 \times 0.15 \times 0.08$

$1.343 \mathrm{~g} / \mathrm{cm}^{3}$

1144

$\operatorname{Mo}-\mathrm{K}_{\alpha}(\lambda=0.71069 \AA)$

$5.3-50.76^{\circ}$

$-15 \leq \mathrm{h} \leq 14 ;-12 \leq \mathrm{k} \leq 12 ;-23 \leq 1 \leq 23$

20674

$4759\left(\mathrm{R}_{\mathrm{int}}=0.0465\right)$

$4324(\mathrm{~F}>4 \sigma)$

4759

312

$\mathrm{R}_{1}=0.0730$

$\mathrm{wR}_{2}=0.1702$

$\mathrm{R}_{1}=0.0821$

$\mathrm{wR}_{2}=0.1790$

1.147

$+0.663,-0.493$ 
Table S2. Refined Positional Parameters for (meso)-6

\begin{tabular}{|c|c|c|c|c|}
\hline Atom & $\mathrm{x}$ & $\mathrm{y}$ & $\mathrm{Z}$ & $\mathrm{U}_{\mathrm{eq}}, \AA^{2}$ \\
\hline $\mathrm{Ti}$ & $0.43529(5)$ & $0.53508(6)$ & $0.42547(3)$ & $0.0322(2)$ \\
\hline 01 & $0.5260(2)$ & $0.6346(2)$ & $0.37892(14)$ & $0.0369(6)$ \\
\hline $\mathrm{O} 2$ & $0.5749(2)$ & $0.4606(2)$ & $0.47614(13)$ & $0.0329(6)$ \\
\hline $\mathrm{O} 3$ & $0.3154(2)$ & $0.6140(3)$ & $0.4105(2)$ & $0.0471(7)$ \\
\hline O4 & $0.4137(2)$ & $0.3906(3)$ & $0.3828(2)$ & $0.0424(7)$ \\
\hline C1 & $0.6108(3)$ & $0.6986(3)$ & $0.4074(2)$ & $0.0338(8)$ \\
\hline $\mathrm{C} 2$ & $0.6015(3)$ & $0.8247(4)$ & $0.4207(2)$ & $0.0415(10)$ \\
\hline $\mathrm{H} 2$ & $0.5393(3)$ & $0.8665(4)$ & $0.4085(2)$ & 0.055 \\
\hline C3 & $0.6850(4)$ & $0.8869(4)$ & $0.4520(2)$ & $0.0491(11)$ \\
\hline H3 & $0.6792(4)$ & $0.9720(4)$ & $0.4594(2)$ & 0.065 \\
\hline C4 & $0.7783(4)$ & $0.8271(4)$ & $0.4732(2)$ & $0.0426(10)$ \\
\hline C5 & $0.8640(4)$ & $0.8984(5)$ & $0.5134(3)$ & $0.0618(14)$ \\
\hline $\mathrm{H} 5 \mathrm{a}$ & $0.8759(4)$ & $0.9764(5)$ & $0.4903(3)$ & 0.082 \\
\hline $\mathrm{H} 5 \mathrm{~b}$ & $0.8399(4)$ & $0.9180(5)$ & $0.5580(3)$ & 0.082 \\
\hline C6 & $0.9655(5)$ & $0.8305(6)$ & $0.5225(3)$ & $0.078(2)$ \\
\hline $\mathrm{H} 6 \mathrm{a}$ & $1.0022(5)$ & $0.8367(6)$ & $0.4809(3)$ & 0.104 \\
\hline $\mathrm{H} 6 \mathrm{~b}$ & $1.0090(5)$ & $0.8695(6)$ & $0.5587(3)$ & 0.104 \\
\hline C7 & $0.9514(4)$ & $0.6973(5)$ & $0.5393(3)$ & $0.069(2)$ \\
\hline $\mathrm{H} 7 \mathrm{a}$ & $0.9187(4)$ & $0.6910(5)$ & $0.5824(3)$ & 0.091 \\
\hline $\mathrm{H} 7 \mathrm{~b}$ & $1.0200(4)$ & $0.6579(5)$ & $0.5448(3)$ & 0.091 \\
\hline C8 & $0.8851(3)$ & $0.6289(4)$ & $0.4856(2)$ & $0.0440(10)$ \\
\hline $\mathrm{H} 8 \mathrm{a}$ & $0.8630(3)$ & $0.5504(4)$ & $0.5044(2)$ & 0.059 \\
\hline $\mathrm{H} 8 \mathrm{~b}$ & $0.9278(3)$ & $0.6104(4)$ & $0.4477(2)$ & 0.059 \\
\hline $\mathrm{C} 9$ & $0.7884(3)$ & $0.7000(4)$ & $0.4589(2)$ & $0.0361(9)$ \\
\hline C10 & $0.7059(3)$ & $0.6368(4)$ & $0.4230(2)$ & $0.0331(8)$ \\
\hline C11 & $0.7151(3)$ & $0.5031(3)$ & $0.4047(2)$ & $0.0322(8)$ \\
\hline C12 & $0.7841(3)$ & $0.4610(4)$ & $0.3561(2)$ & $0.0358(9)$ \\
\hline C13 & $0.8441(3)$ & $0.5547(4)$ & $0.3153(2)$ & $0.0447(10)$ \\
\hline $\mathrm{H} 13 \mathrm{a}$ & $0.7968(3)$ & $0.6214(4)$ & $0.3005(2)$ & 0.059 \\
\hline $\mathrm{H} 13 \mathrm{~b}$ & $0.9002(3)$ & $0.5905(4)$ & $0.3447(2)$ & 0.059 \\
\hline C14 & $0.8910(5)$ & $0.4986(6)$ & $0.2534(3)$ & $0.067(2)$ \\
\hline $\mathrm{H} 14 \mathrm{a}$ & $0.8356(5)$ & $0.4856(6)$ & $0.2179(3)$ & 0.089 \\
\hline $\mathrm{H} 14 \mathrm{~b}$ & $0.9414(5)$ & $0.5563(6)$ & $0.2362(3)$ & 0.089 \\
\hline C15 & $0.9438(4)$ & $0.3792(5)$ & $0.2694(3)$ & $0.0645(14)$ \\
\hline $\mathrm{H} 15 \mathrm{a}$ & $0.9997(4)$ & $0.3921(5)$ & $0.3046(3)$ & 0.086 \\
\hline $\mathrm{H} 15 \mathrm{~b}$ & $0.9753(4)$ & $0.3483(5)$ & $0.2291(3)$ & 0.086 \\
\hline C16 & $0.8677(4)$ & $0.2836(5)$ & $0.2936(2)$ & $0.0526(12)$ \\
\hline $\mathrm{H} 16 \mathrm{a}$ & $0.9075(4)$ & $0.2154(5)$ & $0.3147(2)$ & 0.070 \\
\hline $\mathrm{H} 16 \mathrm{~b}$ & $0.8268(4)$ & $0.2506(5)$ & $0.2543(2)$ & 0.070 \\
\hline C17 & $0.7929(3)$ & $0.3332(4)$ & $0.3439(2)$ & $0.0398(9)$ \\
\hline C18 & $0.7313(3)$ & $0.2511(4)$ & $0.3779(2)$ & $0.0431(10)$ \\
\hline $\mathrm{H} 18$ & $0.7387(3)$ & $0.1662(4)$ & $0.3704(2)$ & 0.057 \\
\hline C19 & $0.6594(3)$ & $0.2911(4)$ & $0.4223(2)$ & $0.0397(9)$ \\
\hline $\mathrm{H} 19$ & $0.6169(3)$ & $0.2346(4)$ & $0.4435(2)$ & 0.053 \\
\hline C20 & $0.6516(3)$ & $0.4170(3)$ & $0.4349(2)$ & $0.0321(8)$ \\
\hline C21 & $0.2350(4)$ & $0.7043(6)$ & $0.4031(4)$ & $0.088(2)$ \\
\hline $\mathrm{H} 21$ & $0.2110(4)$ & $0.7151(6)$ & $0.4492(4)$ & 0.117 \\
\hline C22 & $0.2831(5)$ & $0.8270(5)$ & $0.3865(4)$ & $0.092(2)$ \\
\hline $\mathrm{H} 22 \mathrm{a}$ & $0.2288(7)$ & $0.8885(12)$ & $0.380(3)$ & 0.137 \\
\hline $\mathrm{H} 22 \mathrm{~b}$ & $0.319(4)$ & $0.8193(13)$ & $0.345(2)$ & 0.137 \\
\hline $\mathrm{H} 22 \mathrm{c}$ & $0.332(3)$ & $0.852(2)$ & $0.4232(12)$ & 0.137 \\
\hline C23 & $0.1450(4)$ & $0.6629(7)$ & $0.3638(5)$ & $0.104(3)$ \\
\hline $\mathrm{H} 23 \mathrm{a}$ & $0.091(2)$ & $0.726(3)$ & $0.363(3)$ & 0.156 \\
\hline
\end{tabular}




\begin{tabular}{|lllll|}
$\mathrm{H} 23 \mathrm{~b}$ & $0.119(3)$ & $0.588(3)$ & $0.383(2)$ & 0.156 \\
$\mathrm{H} 23 \mathrm{c}$ & $0.1633(12)$ & $0.647(5)$ & $0.3180(9)$ & 0.156 \\
$\mathrm{C} 24$ & $0.3324(4)$ & $0.2993(5)$ & $0.3701(3)$ & $0.0633(14)$ \\
$\mathrm{H} 24$ & $0.2926(4)$ & $0.2950(5)$ & $0.4111(3)$ & 0.084 \\
$\mathrm{C} 25$ & $0.2581(4)$ & $0.3381(6)$ & $0.3135(3)$ & $0.076(2)$ \\
$\mathrm{H} 25 \mathrm{a}$ & $0.204(2)$ & $0.277(2)$ & $0.307(2)$ & 0.113 \\
$\mathrm{H} 25 \mathrm{~b}$ & $0.2950(8)$ & $0.347(4)$ & $0.2726(6)$ & 0.113 \\
$\mathrm{H} 25 \mathrm{c}$ & $0.227(3)$ & $0.417(2)$ & $0.3243(10)$ & 0.113 \\
$\mathrm{C} 26$ & $0.3816(5)$ & $0.1768(5)$ & $0.3618(4)$ & $0.090(2)$ \\
$\mathrm{H} 26 \mathrm{a}$ & $0.3277(5)$ & $0.1152(9)$ & $0.353(3)$ & 0.135 \\
$\mathrm{H} 26 \mathrm{~b}$ & $0.423(3)$ & $0.156(2)$ & $0.4026(10)$ & 0.135 \\
$\mathrm{H} 26 \mathrm{c}$ & $0.426(3)$ & $0.1794(14)$ & $0.324(2)$ & 0.135 \\
$\mathrm{C} 27$ & $0.5416(4)$ & $0.4657(5)$ & $0.2442(2)$ & $0.0548(12)$ \\
$\mathrm{H} 27 \mathrm{a}$ & $0.5239(4)$ & $0.3993(5)$ & $0.2748(2)$ & 0.073 \\
$\mathrm{H} 27 \mathrm{~b}$ & $0.5872(4)$ & $0.5237(5)$ & $0.2697(2)$ & 0.073 \\
$\mathrm{Cl} 1$ & $0.60888(12)$ & $0.4036(2)$ & $0.17716(7)$ & $0.0740(4)$ \\
$\mathrm{Cl} 2$ & $0.42660(10)$ & $0.54223(13)$ & $0.21453(7)$ & $0.0639(4)$ \\
\hline &
\end{tabular}


Table S3. Refined Thermal Parameters (U's) for (meso)-6

\begin{tabular}{|c|c|c|c|c|c|c|}
\hline Atom & $\mathrm{U}_{11}$ & $\mathrm{U}_{22}$ & $\mathrm{U}_{33}$ & $\mathrm{U}_{23}$ & $\mathrm{U}_{13}$ & $\mathrm{U}_{12}$ \\
\hline $\mathrm{Ti}$ & $0.0278(4)$ & $0.0331(4)$ & $0.0357(4)$ & $0.0000(3)$ & $0.0017(3)$ & $0.0012(3)$ \\
\hline 01 & $0.0323(14)$ & $0.0353(14)$ & $0.043(2)$ & $0.0032(12)$ & $-0.0001(12)$ & $-0.0014(11)$ \\
\hline $\mathrm{O} 2$ & $0.0261(12)$ & $0.0338(14)$ & $0.0392(14)$ & $0.0003(11)$ & $0.0055(11)$ & $0.0023(10)$ \\
\hline $\mathrm{O} 3$ & $0.035(2)$ & $0.052(2)$ & $0.054(2)$ & $0.0032(14)$ & $0.0000(13)$ & $0.0099(13)$ \\
\hline $\mathrm{O} 4$ & $0.036(2)$ & $0.044(2)$ & $0.047(2)$ & $-0.0070(13)$ & $-0.0026(13)$ & $-0.0068(12)$ \\
\hline $\mathrm{C} 1$ & $0.040(2)$ & $0.030(2)$ & $0.032(2)$ & $0.001(2)$ & $0.008(2)$ & $-0.002(2)$ \\
\hline C2 & $0.052(2)$ & $0.030(2)$ & $0.044(2)$ & $0.003(2)$ & $0.012(2)$ & $0.002(2)$ \\
\hline C3 & $0.067(3)$ & $0.030(2)$ & $0.051(3)$ & $-0.001(2)$ & $0.013(2)$ & $-0.007(2)$ \\
\hline C4 & $0.054(3)$ & $0.037(2)$ & $0.038(2)$ & $-0.003(2)$ & $0.008(2)$ & $-0.015(2)$ \\
\hline C5 & $0.075(4)$ & $0.050(3)$ & $0.060(3)$ & $-0.010(2)$ & $0.000(3)$ & $-0.022(3)$ \\
\hline C6 & $0.066(4)$ & $0.079(4)$ & $0.089(4)$ & $-0.013(3)$ & $-0.008(3)$ & $-0.033(3)$ \\
\hline C7 & $0.057(3)$ & $0.079(4)$ & $0.067(3)$ & $-0.005(3)$ & $-0.011(3)$ & $-0.012(3)$ \\
\hline C8 & $0.031(2)$ & $0.058(3)$ & $0.042(2)$ & $-0.003(2)$ & $-0.006(2)$ & $-0.006(2)$ \\
\hline C9 & $0.038(2)$ & $0.038(2)$ & $0.033(2)$ & $0.000(2)$ & $0.007(2)$ & $-0.008(2)$ \\
\hline C10 & $0.031(2)$ & $0.036(2)$ & $0.033(2)$ & $-0.001(2)$ & $0.006(2)$ & $-0.004(2)$ \\
\hline C11 & $0.028(2)$ & $0.032(2)$ & $0.037(2)$ & $-0.002(2)$ & $0.002(2)$ & $0.001(2)$ \\
\hline C12 & $0.030(2)$ & $0.042(2)$ & $0.035(2)$ & $-0.004(2)$ & $0.003(2)$ & $0.000(2)$ \\
\hline C13 & $0.036(2)$ & $0.058(3)$ & $0.041(2)$ & $0.001(2)$ & $0.010(2)$ & $-0.003(2)$ \\
\hline C14 & $0.065(3)$ & $0.082(4)$ & $0.058(3)$ & $0.001(3)$ & $0.023(3)$ & $-0.002(3)$ \\
\hline C15 & $0.053(3)$ & $0.085(4)$ & $0.058(3)$ & $-0.005(3)$ & $0.021(2)$ & $0.013(3)$ \\
\hline C16 & $0.049(3)$ & $0.061(3)$ & $0.048(3)$ & $-0.019(2)$ & $0.008(2)$ & $0.013(2)$ \\
\hline C17 & $0.032(2)$ & $0.046(2)$ & $0.041(2)$ & $-0.010(2)$ & $0.001(2)$ & $0.004(2)$ \\
\hline C18 & $0.041(2)$ & $0.033(2)$ & $0.055(3)$ & $-0.008(2)$ & $-0.001(2)$ & $0.005(2)$ \\
\hline C19 & $0.039(2)$ & $0.030(2)$ & $0.051(2)$ & $0.001(2)$ & $0.009(2)$ & $0.001(2)$ \\
\hline C20 & $0.028(2)$ & $0.032(2)$ & $0.037(2)$ & $-0.002(2)$ & $0.006(2)$ & $0.003(2)$ \\
\hline C21 & $0.047(3)$ & $0.066(4)$ & $0.145(6)$ & $-0.001(4)$ & $-0.033(4)$ & $0.022(3)$ \\
\hline $\mathrm{C} 22$ & $0.088(4)$ & $0.053(3)$ & $0.128(6)$ & $-0.004(4)$ & $-0.036(4)$ & $0.018(3)$ \\
\hline $\mathrm{C} 23$ & $0.044(3)$ & $0.102(5)$ & $0.162(7)$ & $0.040(5)$ & $-0.018(4)$ & $0.009(3)$ \\
\hline C24 & $0.058(3)$ & $0.059(3)$ & $0.073(3)$ & $-0.011(3)$ & $0.001(3)$ & $-0.023(2)$ \\
\hline $\mathrm{C} 25$ & $0.057(3)$ & $0.086(4)$ & $0.080(4)$ & $-0.009(3)$ & $-0.019(3)$ & $-0.025(3)$ \\
\hline C26 & $0.090(5)$ & $0.046(3)$ & $0.133(6)$ & $-0.016(3)$ & $-0.001(4)$ & $-0.013(3)$ \\
\hline C27 & $0.061(3)$ & $0.058(3)$ & $0.046(3)$ & $0.007(2)$ & $0.008(2)$ & $-0.001(2)$ \\
\hline Cl1 & $0.0784(10)$ & $0.0807(10)$ & $0.0633(8)$ & $-0.0203(7)$ & $0.0082(7)$ & $0.0095(8)$ \\
\hline $\mathrm{Cl} 2$ & $0.0467(7)$ & $0.0703(8)$ & $0.0743(9)$ & $0.0102(7)$ & $-0.0004(6)$ & $-0.0047(6)$ \\
\hline
\end{tabular}


Table S4. Bond Distances in (meso)-6, $\AA$

\begin{tabular}{|llllll|}
\hline Ti-O3 & $1.756(3)$ & Ti-O4 & $1.779(3)$ & Ti-O1 & $1.858(3)$ \\
Ti-O2 & $1.947(3)$ & Ti-O2 & $2.138(3)$ & Ti-Ti & $3.3552(14)$ \\
O1-C1 & $1.369(5)$ & O2-C20 & $1.390(4)$ & O2-Ti & $1.947(3)$ \\
O3-C21 & $1.412(6)$ & O4-C24 & $1.437(5)$ & C1-C2 & $1.388(5)$ \\
C1-C10 & $1.399(5)$ & C2-C3 & $1.368(6)$ & C3-C4 & $1.392(7)$ \\
C4-C9 & $1.403(6)$ & C4-C5 & $1.516(6)$ & C5-C6 & $1.485(8)$ \\
C6-C7 & $1.484(8)$ & C7-C8 & $1.502(6)$ & C8-C9 & $1.515(6)$ \\
C9-C10 & $1.404(5)$ & C10-C11 & $1.489(5)$ & C11-C20 & $1.387(5)$ \\
C11-C12 & $1.413(5)$ & C12-C17 & $1.401(6)$ & C12-C13 & $1.523(6)$ \\
C13-C14 & $1.514(6)$ & C14-C15 & $1.475(8)$ & C15-C16 & $1.511(7)$ \\
C16-C17 & $1.512(5)$ & C17-C18 & $1.382(6)$ & C18-C19 & $1.375(6)$ \\
C19-C20 & $1.381(5)$ & C21-C23 & $1.412(9)$ & C21-C22 & $1.500(9)$ \\
C24-C26 & $1.473(8)$ & C24-C25 & $1.474(8)$ & C27-Cl2 & $1.748(5)$ \\
C27-Cl1 & $1.748(5)$ & & & & \\
\hline
\end{tabular}

Table S5. Bond Angles in (meso)-6, ${ }^{\circ}$

\begin{tabular}{|llllll|}
\hline O3-Ti-O4 & $103.67(14)$ & O3-Ti-O1 & $101.55(13)$ & O4-Ti-O1 & $110.70(13)$ \\
O3-Ti-O2 & $92.49(13)$ & O4-Ti-O2 & $118.09(12)$ & O1-Ti-O2 & $123.93(12)$ \\
O3-Ti-O2 & $161.49(13)$ & O4-Ti-O2 & $89.51(12)$ & O1-Ti-O2 & $85.43(11)$ \\
O2-Ti-O2 & $69.63(11)$ & O3-Ti-Ti & $129.00(11)$ & O4-Ti-Ti & $105.54(10)$ \\
O1-Ti-Ti & $105.85(9)$ & O2-Ti-Ti & $36.67(7)$ & O2-Ti-Ti & $32.96(7)$ \\
C1-O1-Ti & $125.9(2)$ & C20-O2-Ti & $132.9(2)$ & C20-O2-Ti & $116.7(2)$ \\
Ti-O2-Ti & $110.37(11)$ & C21-O3-Ti & $165.2(3)$ & C24-O4-Ti & $140.1(3)$ \\
O1-C1-C2 & $119.7(4)$ & O1-C1-C10 & $120.0(3)$ & C2-C1-C10 & $120.4(4)$ \\
C3-C2-C1 & $119.4(4)$ & C2-C3-C4 & $122.1(4)$ & C3-C4-C9 & $118.6(4)$ \\
C3-C4-C5 & $119.7(4)$ & C9-C4-C5 & $121.7(4)$ & C6-C5-C4 & $113.9(4)$ \\
C7-C6-C5 & $112.7(5)$ & C6-C7-C8 & $112.8(5)$ & C7-C8-C9 & $113.8(4)$ \\
C4-C9-C10 & $119.9(4)$ & C4-C9-C8 & $120.4(4)$ & C10-C9-C8 & $119.7(4)$ \\
C1-C10-C9 & $119.3(4)$ & C1-C10-C11 & $119.1(3)$ & C9-C10-C11 & $121.5(3)$ \\
C20-C11-C12 & $118.6(3)$ & C20-C11-C10 & $119.0(3)$ & C12-C11-C10 & $122.3(3)$ \\
C17-C12-C11 & $119.4(4)$ & C17-C12-C13 & $120.7(3)$ & C11-C12-C13 & $119.9(3)$ \\
C14-C13-C12 & $113.3(4)$ & C15-C14-C13 & $111.9(4)$ & C14-C15-C16 & $111.5(4)$ \\
C15-C16-C17 & $114.1(4)$ & C18-C17-C12 & $119.4(4)$ & C18-C17-C16 & $119.3(4)$ \\
C12-C17-C16 & $121.3(4)$ & C19-C18-C17 & $121.9(4)$ & C18-C19-C20 & $118.6(4)$ \\
C19-C20-C11 & $121.9(4)$ & C19-C20-O2 & $119.8(3)$ & C11-C20-O2 & $118.3(3)$ \\
O3-C21-C23 & $113.3(5)$ & O3-C21-C22 & $108.9(5)$ & C23-C21-C22 & $119.1(6)$ \\
O4-C24-C26 & $108.9(4)$ & O4-C24-C25 & $111.0(4)$ & C26-C24-C25 & $115.2(5)$ \\
Cl2-C27-Cl1 & $111.7(3)$ & & & & \\
\hline
\end{tabular}


Ortep of (meso)-7 with full atom labeling

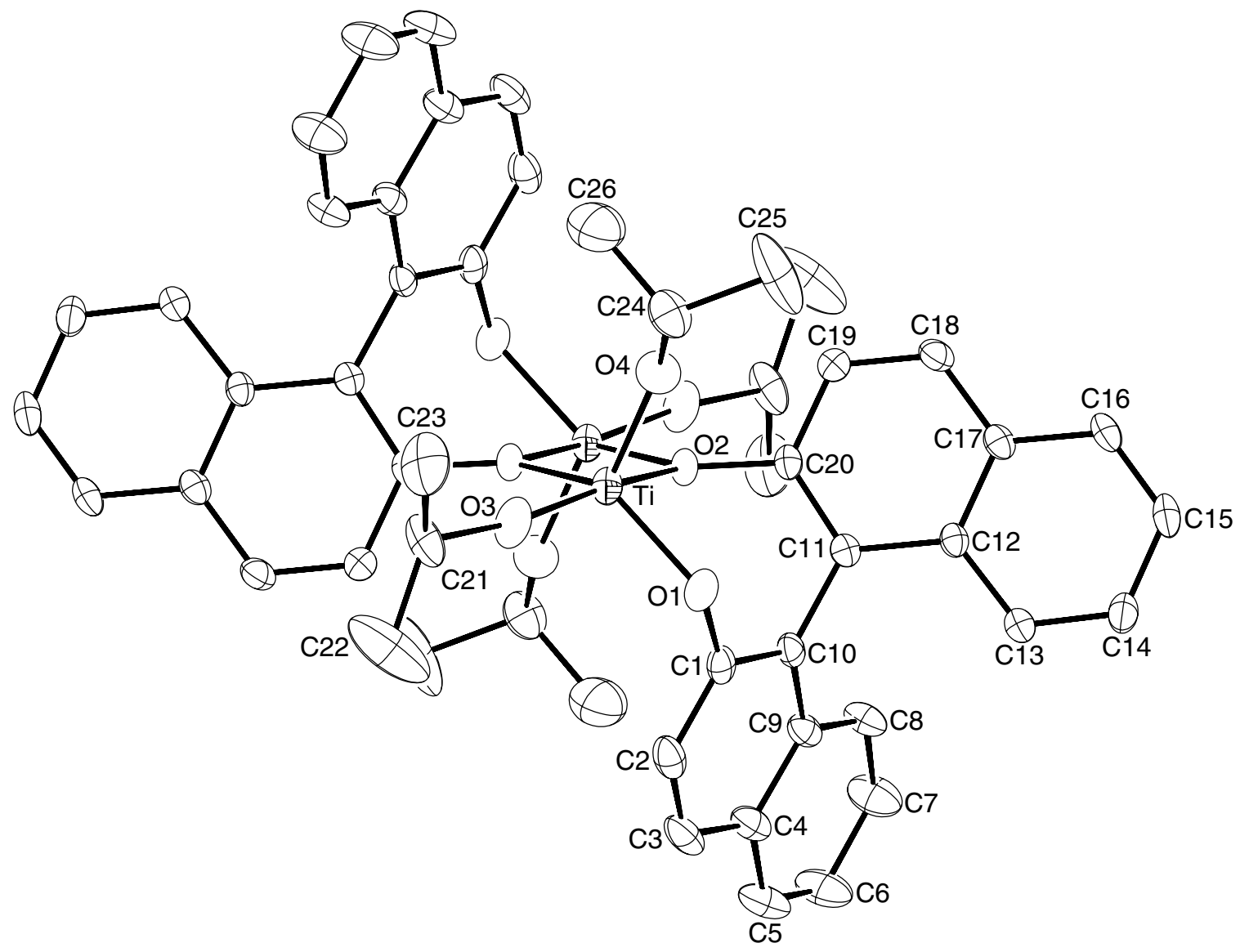


Table S6. Summary of Structure Determination of (meso)-7

Formula:

Formula weight:

Crystal class:

Space group:

Z

Cell constants:

a

b

c

$\alpha$

$\beta$

$\gamma$

$\mathrm{V}$

$\mu$

crystal size, $\mathrm{mm}$

$\mathrm{D}_{\text {calc }}$

$\mathrm{F}(000)$

Radiation:

$2 \theta$ range

hkl collected:

No. reflections measured:

No. unique reflections:

No. observed reflections

No. reflections used in refinement

No. parameters

$R$ indices $(F>4 \sigma)$

$\mathrm{R}$ indices (all data)

GOF:

Final Difference Peaks, e/ $\AA^{3}$
$\mathrm{Ti}_{2} \mathrm{C}_{52} \mathrm{H}_{52} \mathrm{O}_{8}$

900.74

triclinic

$\mathrm{P} \overline{1}(\# 2)$

1

$9.003(3) \AA$

$11.276(4) \AA$

$12.647(5) \AA$

$111.534(6)^{\circ}$

$100.223(5)^{\circ}$

$100.738(5)^{\circ}$

1130.1(7) $\AA^{3}$

$4.08 \mathrm{~cm}^{-1}$

$0.18 \times 0.12 \times 0.08$

$1.324 \mathrm{~g} / \mathrm{cm}^{3}$

472

$\operatorname{Mo}-\mathrm{K}_{\alpha}(\lambda=0.71069 \AA)$

$5.16-54.92^{\circ}$

$-10 \leq \mathrm{h} \leq 9 ;-11 \leq \mathrm{k} \leq 14 ;-14 \leq 1 \leq 15$

7397

$4412\left(\mathrm{R}_{\mathrm{int}}=0.0285\right)$

$3262(\mathrm{~F}>4 \sigma)$

4412

334

$\mathrm{R}_{1}=0.0707$

$\mathrm{wR}_{2}=0.1604$

$\mathrm{R}_{1}=0.1007$

$\mathrm{wR}_{2}=0.1767$

1.054

$+0.545,-0.377$ 
Table S7. Refined Positional Parameters for (meso)-7

\begin{tabular}{|c|c|c|c|c|}
\hline Atom & $\mathrm{x}$ & $\mathrm{y}$ & $\mathrm{Z}$ & $\mathrm{U}_{\mathrm{eq}}, \AA^{2}$ \\
\hline $\mathrm{Ti}$ & $0.32390(8)$ & $0.49847(7)$ & $0.52959(6)$ & $0.0358(2)$ \\
\hline 01 & $0.2054(3)$ & $0.5474(3)$ & $0.4242(2)$ & $0.0403(6)$ \\
\hline $\mathrm{O} 2$ & $0.4487(3)$ & $0.4461(2)$ & $0.3977(2)$ & $0.0354(6)$ \\
\hline $\mathrm{O} 3$ & $0.2813(3)$ & $0.5897(3)$ & $0.6606(2)$ & $0.0546(8)$ \\
\hline $\mathrm{O} 4$ & $0.2374(3)$ & $0.3308(3)$ & $0.4967(3)$ & $0.0597(8)$ \\
\hline C1 & $0.2642(4)$ & $0.6266(4)$ & $0.3746(3)$ & $0.0339(8)$ \\
\hline $\mathrm{C} 2$ & $0.2771(4)$ & $0.7626(4)$ & $0.4296(3)$ & $0.0447(10)$ \\
\hline $\mathrm{H} 2$ & $0.2433(4)$ & $0.7946(4)$ & $0.4974(3)$ & 0.059 \\
\hline C3 & $0.3378(5)$ & $0.8485(4)$ & $0.3859(4)$ & $0.0504(11)$ \\
\hline $\mathrm{H} 3$ & $0.3424(5)$ & $0.9378(4)$ & $0.4226(4)$ & 0.067 \\
\hline C4 & $0.3942(4)$ & $0.8029(4)$ & $0.2847(4)$ & $0.0435(9)$ \\
\hline C5 & $0.4658(6)$ & $0.8907(4)$ & $0.2398(5)$ & $0.0617(13)$ \\
\hline H5 & $0.4708(6)$ & $0.9801(4)$ & $0.2755(5)$ & 0.082 \\
\hline C6 & $0.5266(6)$ & $0.8484(4)$ & $0.1472(5)$ & $0.0677(14)$ \\
\hline $\mathrm{H} 6$ & $0.5730(6)$ & $0.9082(4)$ & $0.1199(5)$ & 0.090 \\
\hline C7 & $0.5194(6)$ & $0.7136(4)$ & $0.0920(5)$ & $0.0637(13)$ \\
\hline $\mathrm{H} 7$ & $0.5626(6)$ & $0.6844(4)$ & $0.0287(5)$ & 0.085 \\
\hline C8 & $0.4494(5)$ & $0.6249(4)$ & $0.1308(4)$ & $0.0437(9)$ \\
\hline $\mathrm{H} 8$ & $0.4456(5)$ & $0.5360(4)$ & $0.0929(4)$ & 0.058 \\
\hline $\mathrm{C} 9$ & $0.3827(4)$ & $0.6651(3)$ & $0.2268(3)$ & $0.0351(8)$ \\
\hline C10 & $0.3123(4)$ & $0.5747(3)$ & $0.2716(3)$ & $0.0316(8)$ \\
\hline C11 & $0.2952(4)$ & $0.4289(3)$ & $0.2154(3)$ & $0.0277(7)$ \\
\hline C12 & $0.2009(4)$ & $0.3432(3)$ & $0.0969(3)$ & $0.0290(7)$ \\
\hline C13 & $0.1198(4)$ & $0.3918(4)$ & $0.0217(3)$ & $0.0352(8)$ \\
\hline $\mathrm{H} 13$ & $0.1293(4)$ & $0.4822(4)$ & $0.0483(3)$ & 0.047 \\
\hline C14 & $0.0276(4)$ & $0.3071(4)$ & $-0.0898(3)$ & $0.0434(9)$ \\
\hline $\mathrm{H} 14$ & $-0.0237(4)$ & $0.3409(4)$ & $-0.1378(3)$ & 0.058 \\
\hline C15 & $0.0100(5)$ & $0.1700(4)$ & $-0.1320(3)$ & $0.0456(10)$ \\
\hline $\mathrm{H} 15$ & $-0.0543(5)$ & $0.1134(4)$ & $-0.2070(3)$ & 0.061 \\
\hline C16 & $0.0871(4)$ & $0.1202(4)$ & $-0.0630(3)$ & $0.0390(9)$ \\
\hline $\mathrm{H} 16$ & $0.0772(4)$ & $0.0296(4)$ & $-0.0922(3)$ & 0.052 \\
\hline C17 & $0.1824(4)$ & $0.2041(3)$ & $0.0529(3)$ & $0.0315(7)$ \\
\hline C18 & $0.2605(4)$ & $0.1519(3)$ & $0.1255(3)$ & $0.0380(8)$ \\
\hline $\mathrm{H} 18$ & $0.2516(4)$ & $0.0615(3)$ & $0.0964(3)$ & 0.050 \\
\hline C19 & $0.3479(4)$ & $0.2325(4)$ & $0.2372(3)$ & $0.0367(8)$ \\
\hline $\mathrm{H} 19$ & $0.3976(4)$ & $0.1969(4)$ & $0.2843(3)$ & 0.049 \\
\hline $\mathrm{C} 20$ & $0.3642(4)$ & $0.3705(3)$ & $0.2826(3)$ & $0.0309(7)$ \\
\hline C21 & $0.2702(11)$ & $0.6600(6)$ & $0.7773(5)$ & $0.055(2)$ \\
\hline $\mathrm{H} 21$ & $0.3746(11)$ & $0.6675(6)$ & $0.8224(5)$ & 0.066 \\
\hline C22 & $0.283(3)$ & $0.8048(10)$ & $0.810(2)$ & $0.141(9)$ \\
\hline $\mathrm{H} 22 \mathrm{a}$ & $0.356(3)$ & $0.8392(10)$ & $0.775(2)$ & 0.211 \\
\hline $\mathrm{H} 22 \mathrm{~b}$ & $0.319(3)$ & $0.8524(10)$ & $0.895(2)$ & 0.211 \\
\hline $\mathrm{H} 22 \mathrm{c}$ & $0.181(3)$ & $0.8150(10)$ & $0.783(2)$ & 0.211 \\
\hline C23 & $0.1717(9)$ & $0.5627(7)$ & $0.8111(6)$ & $0.084(2)$ \\
\hline $\mathrm{H} 23 \mathrm{a}$ & $0.1899(9)$ & $0.4774(7)$ & $0.7759(6)$ & 0.126 \\
\hline $\mathrm{H} 23 \mathrm{~b}$ & $0.0626(9)$ & $0.5558(7)$ & $0.7834(6)$ & 0.126 \\
\hline $\mathrm{H} 23 \mathrm{c}$ & $0.2000(9)$ & $0.5933(7)$ & $0.8954(6)$ & 0.126 \\
\hline $\mathrm{C} 21^{\prime}$ & $0.187(2)$ & $0.6195(11)$ & $0.7416(11)$ & $0.089(5)$ \\
\hline $\mathrm{H} 21^{\prime}$ & $0.089(2)$ & $0.5502(11)$ & $0.7189(11)$ & 0.107 \\
\hline C22' & $0.165(2)$ & $0.7538(11)$ & $0.760(2)$ & $0.119(5)$ \\
\hline H22a' & $0.102(2)$ & $0.7492(11)$ & $0.688(2)$ & 0.179 \\
\hline H22b' & $0.265(2)$ & $0.8164(11)$ & $0.782(2)$ & 0.179 \\
\hline $\mathrm{H}^{2} 2 \mathrm{c}^{\prime}$ & $0.113(2)$ & $0.7816(11)$ & $0.822(2)$ & 0.179 \\
\hline
\end{tabular}




\begin{tabular}{|c|c|c|c|c|}
\hline C23' & $0.287(3)$ & $0.661(4)$ & $0.866(2)$ & $0.108(7)$ \\
\hline H23a' & $0.315(3)$ & $0.586(4)$ & $0.873(2)$ & 0.163 \\
\hline H23b' & $0.228(3)$ & $0.694(4)$ & $0.921(2)$ & 0.163 \\
\hline H23c' & $0.380(3)$ & $0.729(4)$ & $0.882(2)$ & 0.163 \\
\hline C24 & $0.1215(6)$ & $0.2451(5)$ & $0.5178(5)$ & $0.0571(14)$ \\
\hline H24 & $0.0499(6)$ & $0.2911(5)$ & $0.5552(5)$ & 0.068 \\
\hline C25 & $0.034(2)$ & $0.127(2)$ & $0.4023(12)$ & $0.125(7)$ \\
\hline $\mathrm{H} 25 \mathrm{a}$ & $-0.027(2)$ & $0.155(2)$ & $0.3511(12)$ & 0.187 \\
\hline $\mathrm{H} 25 \mathrm{~b}$ & $-0.033(2)$ & $0.062(2)$ & $0.4174(12)$ & 0.187 \\
\hline $\mathrm{H} 25 \mathrm{c}$ & $0.109(2)$ & $0.089(2)$ & $0.3652(12)$ & 0.187 \\
\hline C26 & $0.220(3)$ & $0.201(3)$ & $0.599(2)$ & $0.096(6)$ \\
\hline $\mathrm{H} 26 \mathrm{a}$ & $0.275(3)$ & $0.276(3)$ & $0.672(2)$ & 0.144 \\
\hline H26b & $0.294(3)$ & $0.163(3)$ & $0.562(2)$ & 0.144 \\
\hline H26c & $0.153(3)$ & $0.136(3)$ & $0.615(2)$ & 0.144 \\
\hline C24' & $0.230(2)$ & $0.2158(10)$ & $0.5211(12)$ & $0.061(5)$ \\
\hline H24' & $0.328(2)$ & $0.1891(10)$ & $0.5226(12)$ & 0.074 \\
\hline C25' & $0.093(3)$ & $0.113(2)$ & $0.421(2)$ & $0.100(9)$ \\
\hline H25a' & $0.119(3)$ & $0.096(2)$ & $0.347(2)$ & 0.150 \\
\hline H25b' & $0.002(3)$ & $0.147(2)$ & $0.420(2)$ & 0.150 \\
\hline H25c' & $0.071(3)$ & $0.033(2)$ & $0.432(2)$ & 0.150 \\
\hline C26' & $0.181(2)$ & $0.238(2)$ & $0.6341(10)$ & $0.081(4)$ \\
\hline H26a' & $0.264(2)$ & $0.303(2)$ & $0.7002(10)$ & 0.122 \\
\hline H26b' & $0.157(2)$ & $0.157(2)$ & $0.6429(10)$ & 0.122 \\
\hline $\mathrm{H} 26 \mathrm{c}^{\prime}$ & $0.089(2)$ & $0.271(2)$ & $0.6306(10)$ & 0.122 \\
\hline
\end{tabular}


Table S8. Refined Thermal Parameters (U's) for (meso)-7

\begin{tabular}{|c|c|c|c|c|c|c|}
\hline Atom & $\mathrm{U}_{11}$ & $\mathrm{U}_{22}$ & $\mathrm{U}_{33}$ & $\mathrm{U}_{23}$ & $\mathrm{U}_{13}$ & $\mathrm{U}_{12}$ \\
\hline $\mathrm{Ti}$ & $0.0335(4)$ & $0.0393(4)$ & $0.0291(3)$ & $0.0121(3)$ & $0.0054(3)$ & $0.0044(3)$ \\
\hline 01 & $0.0341(14)$ & $0.058(2)$ & $0.0316(13)$ & $0.0184(12)$ & $0.0118(11)$ & $0.0176(12)$ \\
\hline $\mathrm{O} 2$ & $0.0359(13)$ & $0.0385(14)$ & $0.0237(12)$ & $0.0072(11)$ & $0.0017(10)$ & $0.0099(11)$ \\
\hline O3 & $0.049(2)$ & $0.085(2)$ & $0.035(2)$ & $0.024(2)$ & $0.0169(13)$ & $0.025(2)$ \\
\hline $\mathrm{O} 4$ & $0.055(2)$ & $0.050(2)$ & $0.067(2)$ & $0.029(2)$ & $0.010(2)$ & $-0.0043(14)$ \\
\hline C1 & $0.023(2)$ & $0.041(2)$ & $0.029(2)$ & $0.008(2)$ & $0.0007(13)$ & $0.009(2)$ \\
\hline $\mathrm{C} 2$ & $0.037(2)$ & $0.045(2)$ & $0.037(2)$ & $0.000(2)$ & $0.007(2)$ & $0.016(2)$ \\
\hline C3 & $0.042(2)$ & $0.031(2)$ & $0.058(3)$ & $-0.002(2)$ & $0.008(2)$ & $0.014(2)$ \\
\hline C4 & $0.037(2)$ & $0.029(2)$ & $0.056(2)$ & $0.011(2)$ & $0.010(2)$ & $0.006(2)$ \\
\hline C5 & $0.065(3)$ & $0.029(2)$ & $0.089(4)$ & $0.021(2)$ & $0.024(3)$ & $0.011(2)$ \\
\hline C6 & $0.080(3)$ & $0.038(2)$ & $0.096(4)$ & $0.033(3)$ & $0.043(3)$ & $0.010(2)$ \\
\hline C7 & $0.073(3)$ & $0.044(3)$ & $0.084(4)$ & $0.028(2)$ & $0.045(3)$ & $0.012(2)$ \\
\hline C8 & $0.049(2)$ & $0.027(2)$ & $0.055(2)$ & $0.014(2)$ & $0.022(2)$ & $0.008(2)$ \\
\hline C9 & $0.029(2)$ & $0.028(2)$ & $0.041(2)$ & $0.010(2)$ & $0.006(2)$ & $0.0038(14)$ \\
\hline C10 & $0.026(2)$ & $0.030(2)$ & $0.028(2)$ & $0.0051(14)$ & $0.0003(13)$ & $0.0043(14)$ \\
\hline C11 & $0.023(2)$ & $0.029(2)$ & $0.029(2)$ & $0.0103(14)$ & $0.0083(13)$ & $0.0033(13)$ \\
\hline C12 & $0.028(2)$ & $0.030(2)$ & $0.025(2)$ & $0.0087(14)$ & $0.0079(13)$ & $0.0035(14)$ \\
\hline C13 & $0.036(2)$ & $0.030(2)$ & $0.032(2)$ & $0.008(2)$ & $0.005(2)$ & $0.006(2)$ \\
\hline C14 & $0.044(2)$ & $0.047(2)$ & $0.029(2)$ & $0.011(2)$ & $0.000(2)$ & $0.011(2)$ \\
\hline C15 & $0.046(2)$ & $0.043(2)$ & $0.027(2)$ & $0.002(2)$ & $-0.002(2)$ & $0.001(2)$ \\
\hline C16 & $0.039(2)$ & $0.027(2)$ & $0.034(2)$ & $0.002(2)$ & $0.004(2)$ & $-0.002(2)$ \\
\hline C17 & $0.027(2)$ & $0.028(2)$ & $0.033(2)$ & $0.0086(14)$ & $0.0091(14)$ & $-0.0004(14)$ \\
\hline C18 & $0.045(2)$ & $0.025(2)$ & $0.039(2)$ & $0.011(2)$ & $0.010(2)$ & $0.004(2)$ \\
\hline C19 & $0.039(2)$ & $0.035(2)$ & $0.034(2)$ & $0.014(2)$ & $0.005(2)$ & $0.012(2)$ \\
\hline C20 & $0.028(2)$ & $0.032(2)$ & $0.026(2)$ & $0.0083(14)$ & $0.0034(13)$ & $0.0028(14)$ \\
\hline C21 & $0.061(5)$ & $0.051(4)$ & $0.037(4)$ & $0.000(4)$ & $0.033(4)$ & $-0.001(4)$ \\
\hline $\mathrm{C} 22$ & $0.106(14)$ & $0.059(7)$ & $0.22(2)$ & $-0.002(12)$ & $0.09(2)$ & $0.014(10)$ \\
\hline C23 & $0.089(5)$ & $0.108(5)$ & $0.055(4)$ & $0.038(4)$ & $0.038(4)$ & $0.002(5)$ \\
\hline $\mathrm{C} 21^{\prime}$ & $0.053(8)$ & $0.069(7)$ & $0.083(9)$ & $-0.018(8)$ & $0.012(8)$ & $-0.012(7)$ \\
\hline C22' & $0.141(11)$ & $0.069(7)$ & $0.188(14)$ & $0.057(9)$ & $0.106(11)$ & $0.052(7)$ \\
\hline C23' & $0.06(2)$ & $0.14(2)$ & $0.083(13)$ & $0.02(2)$ & $0.035(13)$ & $-0.01(2)$ \\
\hline C24 & $0.043(3)$ & $0.051(3)$ & $0.062(4)$ & $0.015(3)$ & $0.015(3)$ & $-0.004(3)$ \\
\hline C25 & $0.079(9)$ & $0.094(10)$ & $0.103(9)$ & $-0.042(8)$ & $0.012(7)$ & $-0.018(7)$ \\
\hline C26 & $0.10(2)$ & $0.070(13)$ & $0.111(12)$ & $0.041(12)$ & $0.019(12)$ & $0.013(10)$ \\
\hline C24' & $0.054(11)$ & $0.061(11)$ & $0.085(9)$ & $0.038(10)$ & $0.043(9)$ & $0.012(9)$ \\
\hline C25' & $0.14(2)$ & $0.046(9)$ & $0.082(9)$ & $0.028(8)$ & $0.020(10)$ & $-0.029(11)$ \\
\hline C26' & $0.092(9)$ & $0.079(11)$ & $0.079(6)$ & $0.044(7)$ & $0.032(6)$ & $0.008(6)$ \\
\hline
\end{tabular}


Table S9. Bond Distances in (meso)-7, ^

\begin{tabular}{|llllll|}
\hline Ti-O3 & $1.753(3)$ & Ti-O4 & $1.764(3)$ & Ti-O1 & $1.864(3)$ \\
Ti-O2' & $1.965(3)$ & Ti-O2 & $2.140(2)$ & Ti-Ti* $^{*}$ & $3.382(2)$ \\
O1-C1 & $1.351(4)$ & O2-C20 & $1.371(4)$ & O2-Ti* $^{*}$ & $1.965(2)$ \\
O3-C21 & $1.428(4)$ & O3-C21' & $1.429(5)$ & O4-C24 & $1.416(4)$ \\
O4-C24' & $1.432(5)$ & C1-C10 & $1.400(5)$ & C1-C2 & $1.404(5)$ \\
C2-C3 & $1.358(6)$ & C3-C4 & $1.416(6)$ & C4-C5 & $1.422(6)$ \\
C4-C9 & $1.428(5)$ & C5-C6 & $1.347(7)$ & C6-C7 & $1.404(6)$ \\
C7-C8 & $1.368(6)$ & C8-C9 & $1.413(5)$ & C9-C10 & $1.440(5)$ \\
C10-C11 & $1.497(5)$ & C11-C20 & $1.385(5)$ & C11-C12 & $1.442(4)$ \\
C12-C13 & $1.423(5)$ & C12-C17 & $1.424(5)$ & C13-C14 & $1.375(5)$ \\
C14-C15 & $1.404(6)$ & C15-C16 & $1.360(5)$ & C16-C17 & $1.418(5)$ \\
C17-C18 & $1.416(5)$ & C18-C19 & $1.355(5)$ & C19-C20 & $1.414(5)$ \\
C21-C22 & $1.506(5)$ & C21-C23 & $1.512(5)$ & C21'-C22' & $1.503(5)$ \\
C21'-C23' & $1.517(5)$ & C24-C25 & $1.516(5)$ & C24-C26 & $1.518(5)$ \\
C24'-C26' & $1.518(5)$ & C24'-C25' & $1.518(5)$ & & \\
\hline
\end{tabular}

Table S10. Bond Angles in (meso)-7, ${ }^{\circ}$

\begin{tabular}{|llllll|}
\hline O3-Ti-O4 & $105.2(2)$ & O3-Ti-O1 $^{*}$ & $100.52(13)$ & O4-Ti-O1 & $108.77(14)$ \\
O3-Ti-O2 & $93.91(12)$ & O4-Ti-O2* & $112.64(13)$ & O1-Ti-O2* & $130.38(11)$ \\
O3-Ti-O2 & $159.52(13)$ & O4-Ti-O2 & $92.28(13)$ & O1-Ti-O2 & $83.31(10)$ \\
O2'-Ti-O2 & $69.14(10)$ & O3-Ti-Ti* $^{*}$ & $129.22(10)$ & O4-Ti-Ti* & $104.41(11)$ \\
O1-Ti-Ti* & $107.63(8)$ & O2'-Ti-Ti* $^{*}$ & $36.25(7)$ & O2-Ti-Ti' & $32.89(6)$ \\
C1-O1-Ti & $125.4(2)$ & C20-O2-Ti* $^{*}$ & $130.2(2)$ & C20-O2-Ti & $118.5(2)$ \\
Ti'-O2-Ti & $110.86(10)$ & C21-O3-Ti & $170.0(5)$ & C21'-O3-Ti & $155.6(6)$ \\
C24-O4-Ti & $143.9(3)$ & C24'-O4-Ti & $151.4(7)$ & O1-C1-C10 & $120.9(3)$ \\
O1-C1-C2 & $118.2(3)$ & C10-C1-C2 & $120.9(4)$ & C3-C2-C1 & $121.4(4)$ \\
C2-C3-C4 & $120.3(4)$ & C3-C4-C5 & $122.0(4)$ & C3-C4-C9 & $119.5(4)$ \\
C5-C4-C9 & $118.5(4)$ & C6-C5-C4 & $122.1(4)$ & C5-C6-C7 & $119.6(4)$ \\
C8-C7-C6 & $120.5(4)$ & C7-C8-C9 & $121.6(4)$ & C8-C9-C4 & $117.7(3)$ \\
C8-C9-C10 & $122.9(3)$ & C4-C9-C10 & $119.4(3)$ & C1-C10-C9 & $118.3(3)$ \\
C1-C10-C11 & $119.1(3)$ & C9-C10-C11 & $122.5(3)$ & C20-C11-C12 & $117.6(3)$ \\
C20-C11-C10 & $118.7(3)$ & C12-C11-C10 & $123.6(3)$ & C13-C12-C17 & $117.7(3)$ \\
C13-C12-C11 & $122.3(3)$ & C17-C12-C11 & $120.0(3)$ & C14-C13-C12 & $120.9(3)$ \\
C13-C14-C15 & $120.8(4)$ & C16-C15-C14 & $119.9(3)$ & C15-C16-C17 & $121.1(3)$ \\
C18-C17-C16 & $121.1(3)$ & C18-C17-C12 & $119.3(3)$ & C16-C17-C12 & $119.6(3)$ \\
C19-C18-C17 & $120.6(3)$ & C18-C19-C20 & $120.5(3)$ & O2-C20-C11 & $120.2(3)$ \\
O2-C20-C19 & $117.8(3)$ & C11-C20-C19 & $122.1(3)$ & O3-C21-C22 & $114.3(11)$ \\
O3-C21-C23 & $108.1(5)$ & C22-C21-C23 & $129.5(10)$ & O3-C21'-C22' & $107.4(8)$ \\
O3-C21'-C23' & $109.7(14)$ & C22'-C21'-C23' & $96(2)$ & O4-C24-C25 & $108.8(9)$ \\
O4-C24-C26 & $102.2(12)$ & C25-C24-C26 & $109.8(14)$ & O4-C24'-C26' & $109.5(9)$ \\
O4-C24'-C25' & $102.1(11)$ & C26'-C24'-C25' & $106.5(14)$ & & \\
\hline
\end{tabular}

Ortep of $\mathbf{8}$ with full atom labeling 


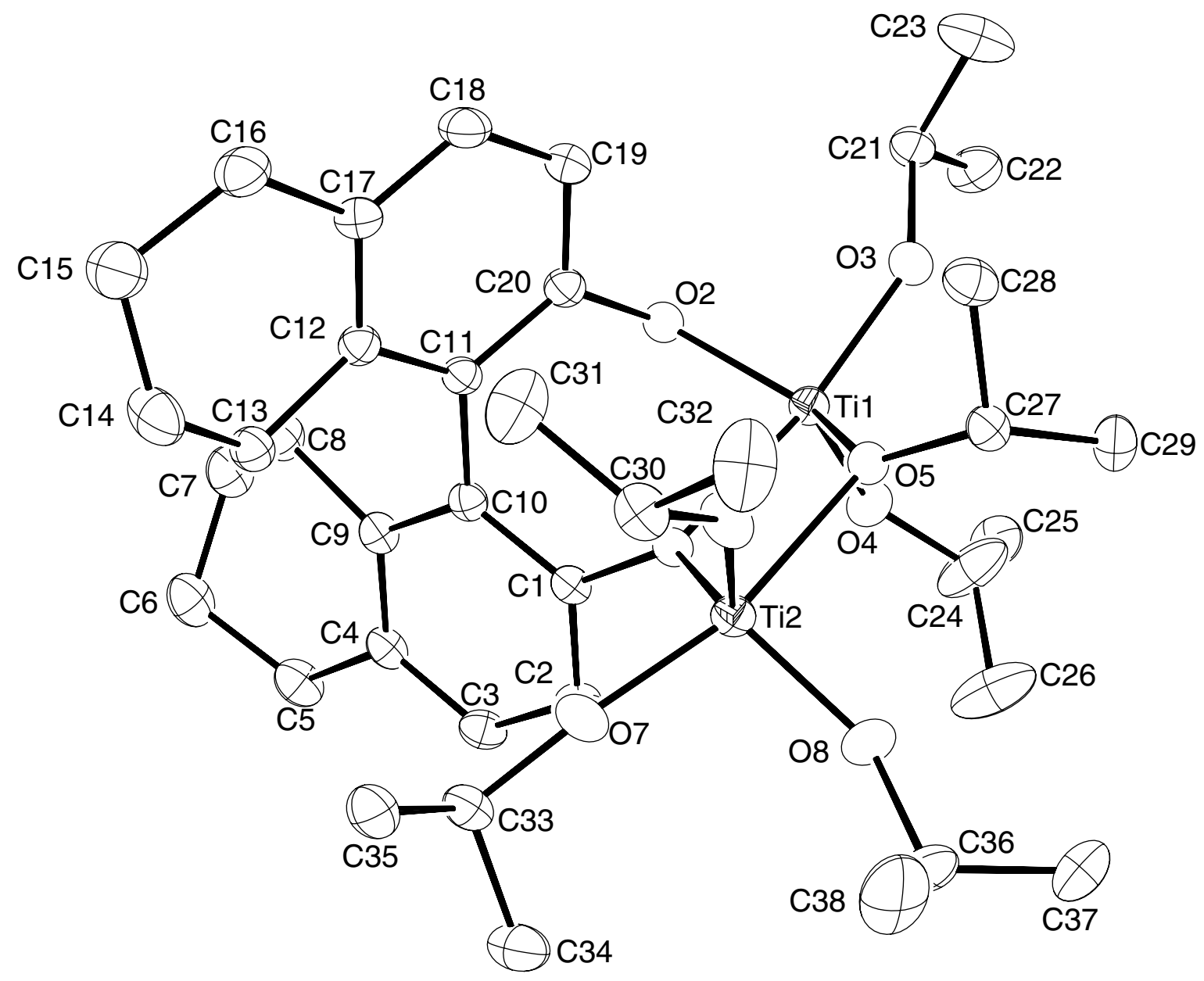


Table S11. Summary of Structure Determination of $\mathbf{8}$

Formula:

Formula weight:

Crystal class:

Space group:

Z

Cell constants:

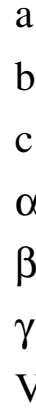

$\mu$

crystal size, $\mathrm{mm}$

$\mathrm{D}_{\text {calc }}$

$\mathrm{F}(000)$

Radiation:

$2 \theta$ range

hkl collected:

No. reflections measured:

No. unique reflections:

No. observed reflections

No. reflections used in refinement

No. parameters

$R$ indices $(F>4 \sigma)$

$\mathrm{R}$ indices (all data)

GOF:

Final Difference Peaks, e/ $/ \AA^{3}$
$\mathrm{Ti}_{2} \mathrm{C}_{38} \mathrm{H}_{62} \mathrm{O}_{8}$

742.68

triclinic

P1̄ (\#2)

2

$12.3281(5) \AA$

$14.1051(6) \AA$

$12.1704(5) \AA$

$91.918(3)^{\circ}$

$100.070(2)^{\circ}$

$80.611(3)^{\circ}$

$2055.8(2) \AA^{3}$

$4.33 \mathrm{~cm}^{-1}$

$0.34 \times 0.20 \times 0.14$

$1.200 \mathrm{~g} / \mathrm{cm}^{3}$

796

$\operatorname{Mo}-K_{\alpha}(\lambda=0.71069 \AA)$

$5.2-50.7^{\circ}$

$-14 \leq \mathrm{h} \leq 14 ;-16 \leq \mathrm{k} \leq 16 ;-14 \leq 1 \leq 14$

16065

$6833\left(\mathrm{R}_{\mathrm{int}}=0.0410\right)$

$5917(\mathrm{~F}>4 \sigma)$

6833

443

$\mathrm{R}_{1}=0.0670$

$\mathrm{wR}_{2}=0.1752$

$\mathrm{R}_{1}=0.0771$

$\mathrm{wR}_{2}=0.1848$

1.099

$+0.502,-0.6$ 
Table S12. Refined Positional Parameters for 8

\begin{tabular}{|c|c|c|c|c|}
\hline Atom & $\mathrm{x}$ & $\mathrm{y}$ & $\mathrm{Z}$ & $\mathrm{U}_{\mathrm{eq}}, \AA^{2}$ \\
\hline Ti1 & $0.38968(5)$ & $0.31255(5)$ & $0.75393(5)$ & $0.0389(2)$ \\
\hline Ti2 & $0.18947(5)$ & $0.24514(5)$ & $0.86839(5)$ & $0.0410(2)$ \\
\hline 01 & $0.2948(2)$ & $0.2017(2)$ & $0.7638(2)$ & $0.0403(5)$ \\
\hline $\mathrm{O} 2$ & $0.3740(2)$ & $0.2801(2)$ & $0.6030(2)$ & $0.0451(6)$ \\
\hline $\mathrm{O} 3$ & $0.4239(2)$ & $0.4296(2)$ & $0.7344(2)$ & $0.0513(7)$ \\
\hline O4 & $0.5166(2)$ & $0.2484(2)$ & $0.8259(2)$ & $0.0523(7)$ \\
\hline O5 & $0.2839(2)$ & $0.3544(2)$ & $0.8523(2)$ & $0.0407(5)$ \\
\hline O6 & $0.0689(2)$ & $0.3362(2)$ & $0.8414(3)$ & $0.0562(7)$ \\
\hline O7 & $0.1239(2)$ & $0.1427(2)$ & $0.8402(2)$ & $0.0548(7)$ \\
\hline O8 & $0.2438(3)$ & $0.2345(2)$ & $1.0141(2)$ & $0.0613(8)$ \\
\hline C1 & $0.3147(3)$ & $0.1154(2)$ & $0.7083(3)$ & $0.0381(7)$ \\
\hline C2 & $0.3702(3)$ & $0.0344(3)$ & $0.7678(3)$ & $0.0464(9)$ \\
\hline $\mathrm{H} 2$ & $0.3907(3)$ & $0.0369(3)$ & $0.8450(3)$ & 0.062 \\
\hline C3 & $0.3944(3)$ & $-0.0504(3)$ & $0.7093(3)$ & $0.0510(9)$ \\
\hline $\mathrm{H} 3$ & $0.4287(3)$ & $-0.1057(3)$ & $0.7488(3)$ & 0.068 \\
\hline C4 & $0.3692(3)$ & $-0.0554(3)$ & $0.5938(3)$ & $0.0455(8)$ \\
\hline C5 & $0.4047(4)$ & $-0.1490(3)$ & $0.5358(4)$ & $0.0634(11)$ \\
\hline $\mathrm{H} 5 \mathrm{a}$ & $0.4855(4)$ & $-0.1611(3)$ & $0.5449(4)$ & 0.084 \\
\hline $\mathrm{H} 5 \mathrm{~b}$ & $0.3818(4)$ & $-0.2008(3)$ & $0.5720(4)$ & 0.084 \\
\hline C6 & $0.3570(4)$ & $-0.1507(3)$ & $0.4125(4)$ & $0.0711(13)$ \\
\hline $\mathrm{H} 6 \mathrm{a}$ & $0.3980(4)$ & $-0.2040(3)$ & $0.3768(4)$ & 0.095 \\
\hline $\mathrm{H} 6 \mathrm{~b}$ & $0.2796(4)$ & $-0.1599(3)$ & $0.4025(4)$ & 0.095 \\
\hline C7 & $0.3646(4)$ & $-0.0588(3)$ & $0.3598(4)$ & $0.0702(13)$ \\
\hline $\mathrm{H} 7 \mathrm{a}$ & $0.3382(4)$ & $-0.0625(3)$ & $0.2801(4)$ & 0.093 \\
\hline $\mathrm{H} 7 \mathrm{~b}$ & $0.4421(4)$ & $-0.0498(3)$ & $0.3708(4)$ & 0.093 \\
\hline $\mathrm{C} 8$ & $0.2965(4)$ & $0.0271(3)$ & $0.4080(3)$ & $0.0542(10)$ \\
\hline $\mathrm{H} 8 \mathrm{a}$ & $0.3163(4)$ & $0.0857(3)$ & $0.3832(3)$ & 0.072 \\
\hline $\mathrm{H} 8 \mathrm{~b}$ & $0.2180(4)$ & $0.0271(3)$ & $0.3794(3)$ & 0.072 \\
\hline C9 & $0.3147(3)$ & $0.0268(3)$ & $0.5343(3)$ & $0.0440(8)$ \\
\hline C10 & $0.2821(3)$ & $0.1122(3)$ & $0.5930(3)$ & $0.0404(8)$ \\
\hline C11 & $0.2176(3)$ & $0.2011(2)$ & $0.5355(3)$ & $0.0394(8)$ \\
\hline C12 & $0.1056(3)$ & $0.2070(3)$ & $0.4822(3)$ & $0.0427(8)$ \\
\hline C13 & $0.0433(3)$ & $0.1241(3)$ & $0.4864(3)$ & $0.0496(9)$ \\
\hline $\mathrm{H} 13 \mathrm{a}$ & $0.0744(3)$ & $0.0719(3)$ & $0.4416(3)$ & 0.066 \\
\hline $\mathrm{H} 13 \mathrm{~b}$ & $0.0561(3)$ & $0.1018(3)$ & $0.5628(3)$ & 0.066 \\
\hline C14 & $-0.0787(4)$ & $0.1455(4)$ & $0.4467(5)$ & $0.080(2)$ \\
\hline $\mathrm{H} 14 \mathrm{a}$ & $-0.1058(4)$ & $0.0854(4)$ & $0.4266(5)$ & 0.107 \\
\hline $\mathrm{H} 14 \mathrm{~b}$ & $-0.1140(4)$ & $0.1733(4)$ & $0.5082(5)$ & 0.107 \\
\hline C15 & $-0.1141(5)$ & $0.2091(4)$ & $0.3536(6)$ & $0.099(2)$ \\
\hline $\mathrm{H} 15 \mathrm{a}$ & $-0.1950(5)$ & $0.2217(4)$ & $0.3382(6)$ & 0.132 \\
\hline $\mathrm{H} 15 \mathrm{~b}$ & $-0.0888(5)$ & $0.1775(4)$ & $0.2885(6)$ & 0.132 \\
\hline C16 & $-0.0708(4)$ & $0.3040(4)$ & $0.3717(4)$ & $0.0674(12)$ \\
\hline $\mathrm{H} 16 \mathrm{a}$ & $-0.1173(4)$ & $0.3462(4)$ & $0.4155(4)$ & 0.090 \\
\hline $\mathrm{H} 16 \mathrm{~b}$ & $-0.0767(4)$ & $0.3342(4)$ & $0.3000(4)$ & 0.090 \\
\hline C17 & $0.0497(3)$ & $0.2928(3)$ & $0.4311(3)$ & $0.0491(9)$ \\
\hline C18 & $0.1044(4)$ & $0.3724(3)$ & $0.4364(4)$ & $0.0578(11)$ \\
\hline $\mathrm{H} 18$ & $0.0671(4)$ & $0.4291(3)$ & $0.4017(4)$ & 0.077 \\
\hline C19 & $0.2126(4)$ & $0.3689(3)$ & $0.4922(3)$ & $0.0539(10)$ \\
\hline $\mathrm{H} 19$ & $0.2474(4)$ & $0.4229(3)$ & $0.4962(3)$ & 0.072 \\
\hline C20 & $0.2691(3)$ & $0.2830(3)$ & $0.5426(3)$ & $0.0428(8)$ \\
\hline C21 & $0.4667(3)$ & $0.4778(3)$ & $0.6555(3)$ & $0.0515(9)$ \\
\hline $\mathrm{H} 21$ & $0.4449(3)$ & $0.4496(3)$ & $0.5819(3)$ & 0.068 \\
\hline $\mathrm{C} 22$ & $0.5914(4)$ & $0.4621(4)$ & $0.6822(4)$ & $0.0691(13)$ \\
\hline
\end{tabular}




\begin{tabular}{|c|c|c|c|c|}
\hline $\mathrm{H} 22 \mathrm{a}$ & $0.6201(4)$ & $0.496(2)$ & $0.629(2)$ & 0.104 \\
\hline $\mathrm{H} 22 \mathrm{~b}$ & $0.6194(4)$ & $0.3947(5)$ & $0.678(3)$ & 0.104 \\
\hline $\mathrm{H} 22 \mathrm{C}$ & $0.6148(4)$ & $0.485(2)$ & $0.7562(12)$ & 0.104 \\
\hline C23 & $0.4166(5)$ & $0.5823(4)$ & $0.6512(6)$ & $0.096(2)$ \\
\hline H23a & $0.447(3)$ & $0.6143(8)$ & $0.598(3)$ & 0.145 \\
\hline $\mathrm{H} 23 \mathrm{~b}$ & $0.434(3)$ & $0.6109(9)$ & $0.7235(11)$ & 0.145 \\
\hline $\mathrm{H} 23 \mathrm{c}$ & $0.3371(7)$ & $0.5886(4)$ & $0.630(4)$ & 0.145 \\
\hline C24 & $0.5959(4)$ & $0.2441(6)$ & $0.9225(4)$ & $0.107(2)$ \\
\hline H24 & $0.5857(4)$ & $0.3118(6)$ & $0.9454(4)$ & 0.142 \\
\hline C25 & $0.7096(4)$ & $0.2317(4)$ & $0.9028(4)$ & $0.0734(13)$ \\
\hline $\mathrm{H} 25 \mathrm{a}$ & $0.7303(11)$ & $0.1677(10)$ & $0.876(3)$ & 0.110 \\
\hline $\mathrm{H} 25 \mathrm{~b}$ & $0.7585(5)$ & $0.242(3)$ & $0.9713(8)$ & 0.110 \\
\hline $\mathrm{H} 25 \mathrm{c}$ & $0.7157(8)$ & $0.277(2)$ & $0.848(2)$ & 0.110 \\
\hline C26 & $0.5678(4)$ & $0.2013(4)$ & $1.0155(4)$ & $0.121(3)$ \\
\hline $\mathrm{H} 26 \mathrm{a}$ & $0.6164(3)$ & $0.2161(9)$ & $1.0822(5)$ & 0.182 \\
\hline $\mathrm{H} 26 \mathrm{~b}$ & $0.5761(10)$ & $0.1329(4)$ & $1.0051(8)$ & 0.182 \\
\hline $\mathrm{H} 26 \mathrm{c}$ & 0.4918(3) & $0.2260(3)$ & $1.0219(3)$ & 0.182 \\
\hline C27 & $0.2607(3)$ & $0.4416(2)$ & $0.9166(3)$ & $0.0443(8)$ \\
\hline $\mathrm{H} 27$ & $0.2103(3)$ & $0.4301(2)$ & $0.9670(3)$ & 0.059 \\
\hline C28 & $0.2010(4)$ & $0.5223(3)$ & $0.8392(4)$ & $0.0587(10)$ \\
\hline $\mathrm{H} 28 \mathrm{a}$ & $0.178(2)$ & $0.5777(8)$ & $0.8824(4)$ & 0.088 \\
\hline $\mathrm{H} 28 \mathrm{~b}$ & $0.137(2)$ & $0.5024(8)$ & $0.794(2)$ & 0.088 \\
\hline $\mathrm{H} 28 \mathrm{c}$ & $0.2505(9)$ & $0.538(2)$ & $0.792(2)$ & 0.088 \\
\hline C29 & $0.3665(4)$ & $0.4636(3)$ & $0.9868(4)$ & $0.0601(11)$ \\
\hline $\mathrm{H} 29 \mathrm{a}$ & $0.3497(5)$ & $0.5186(14)$ & $1.033(2)$ & 0.090 \\
\hline $\mathrm{H} 29 \mathrm{~b}$ & $0.4165(11)$ & $0.477(2)$ & $0.9392(4)$ & 0.090 \\
\hline $\mathrm{H} 29 \mathrm{c}$ & $0.4011(14)$ & $0.4094(9)$ & $1.033(2)$ & 0.090 \\
\hline C30 & $-0.0491(3)$ & $0.3455(3)$ & $0.8135(4)$ & $0.0553(10)$ \\
\hline H30 & $-0.0729(3)$ & $0.2908(3)$ & $0.8448(4)$ & 0.074 \\
\hline C31 & $-0.0837(5)$ & $0.3442(5)$ & $0.6898(5)$ & $0.091(2)$ \\
\hline H31a & $-0.1627(8)$ & $0.344(3)$ & $0.6717(5)$ & 0.136 \\
\hline H31b & $-0.045(3)$ & $0.287(2)$ & $0.6596(6)$ & 0.136 \\
\hline H31c & $-0.066(3)$ & $0.400(2)$ & 0.6586(6) & 0.136 \\
\hline C32 & $-0.0998(4)$ & $0.4364(5)$ & $0.8662(5)$ & $0.092(2)$ \\
\hline H32a & $-0.1796(4)$ & $0.442(2)$ & $0.852(3)$ & 0.139 \\
\hline H32b & $-0.079(3)$ & $0.4908(5)$ & $0.835(3)$ & 0.139 \\
\hline H32c & $-0.073(3)$ & $0.435(2)$ & $0.9452(7)$ & 0.139 \\
\hline C33 & $0.0939(3)$ & $0.0504(3)$ & $0.8210(4)$ & $0.0549(10)$ \\
\hline H33 & $0.1254(3)$ & $0.0216(3)$ & $0.7568(4)$ & 0.073 \\
\hline C34 & $0.1416(4)$ & $-0.0114(4)$ & $0.9203(4)$ & $0.0746(14)$ \\
\hline H34a & $0.124(3)$ & $-0.0749(8)$ & $0.9056(12)$ & 0.112 \\
\hline H34b & $0.110(3)$ & $0.015(2)$ & 0.9837(8) & 0.112 \\
\hline H34c & $0.2211(5)$ & $-0.014(2)$ & $0.936(2)$ & 0.112 \\
\hline C35 & $-0.0314(4)$ & $0.0595(4)$ & $0.7933(4)$ & $0.0687(13)$ \\
\hline H35a & $-0.0518(4)$ & $-0.0026(6)$ & $0.774(3)$ & 0.103 \\
\hline H35b & $-0.0589(5)$ & $0.102(2)$ & $0.731(2)$ & 0.103 \\
\hline H35c & $-0.0634(4)$ & $0.085(3)$ & $0.8568(11)$ & 0.103 \\
\hline C36 & $0.2260(5)$ & $0.2138(4)$ & $1.1222(4)$ & $0.079(2)$ \\
\hline H36 & $0.2498(5)$ & $0.1445(4)$ & $1.1337(4)$ & 0.105 \\
\hline C37 & 0.2994(6) & $0.2632(5)$ & $1.2072(4)$ & $0.095(2)$ \\
\hline H37a & $0.291(3)$ & $0.246(3)$ & $1.2805(4)$ & 0.142 \\
\hline H37b & $0.279(3)$ & $0.3315(5)$ & $1.198(2)$ & 0.142 \\
\hline H37c & $0.3757(6)$ & $0.244(3)$ & 1.198(3) & 0.142 \\
\hline C38 & $0.1078(6)$ & $0.2345(6)$ & $1.1309(5)$ & $0.120(3)$ \\
\hline H38a & $0.0806(13)$ & $0.3015(10)$ & $1.117(5)$ & 0.180 \\
\hline H38b & 0.0984(8) & $0.219(4)$ & $1.204(2)$ & 0.180 \\
\hline H38c & $0.0666(9)$ & $0.197(3)$ & $1.077(3)$ & 0.180 \\
\hline
\end{tabular}




$$
\mathrm{U}_{\mathrm{eq}}=1 /{ }_{3}\left[\mathrm{U}_{11}\left(\mathrm{aa}^{*}\right)^{2}+\mathrm{U}_{22}\left(\mathrm{bb}^{*}\right)^{2}+\mathrm{U}_{33}\left(\mathrm{cc}^{*}\right)^{2}+2 \mathrm{U}_{12} \mathrm{aa} * \mathrm{bb} * \cos \gamma+2 \mathrm{U}_{13} \mathrm{aa} * \mathrm{cc} * \cos \beta+2 \mathrm{U}_{23} \mathrm{bb}^{*} \mathrm{cc} * \cos \alpha\right.
$$


Table S13. Refined Thermal Parameters (U's) for 8

\begin{tabular}{|c|c|c|c|c|c|c|}
\hline Atom & $\mathrm{U}_{11}$ & $\mathrm{U}_{22}$ & $\mathrm{U}_{33}$ & $\mathrm{U}_{23}$ & $\mathrm{U}_{13}$ & $\mathrm{U}_{12}$ \\
\hline Ti1 & $0.0356(3)$ & $0.0440(4)$ & $0.0396(4)$ & $0.0007(3)$ & $0.0118(3)$ & $-0.0081(3)$ \\
\hline $\mathrm{Ti} 2$ & $0.0385(4)$ & $0.0456(4)$ & $0.0425(4)$ & $0.0007(3)$ & $0.0155(3)$ & $-0.0079(3)$ \\
\hline 01 & $0.0431(13)$ & $0.0411(13)$ & $0.0405(12)$ & $-0.0007(11)$ & $0.0140(11)$ & $-0.0106(10)$ \\
\hline $\mathrm{O} 2$ & $0.0442(14)$ & $0.052(2)$ & $0.0440(13)$ & $0.0014(12)$ & $0.0117(11)$ & $-0.0167(11)$ \\
\hline $\mathrm{O} 3$ & $0.059(2)$ & $0.054(2)$ & $0.049(2)$ & $-0.0025(13)$ & $0.0218(13)$ & $-0.0200(13)$ \\
\hline $\mathrm{O} 4$ & $0.0391(14)$ & $0.064(2)$ & $0.051(2)$ & $0.0023(13)$ & $0.0068(11)$ & $-0.0007(12)$ \\
\hline O5 & $0.0413(13)$ & $0.0430(13)$ & $0.0401(13)$ & $-0.0037(11)$ & $0.0127(10)$ & $-0.0084(10)$ \\
\hline O6 & $0.0385(14)$ & $0.051(2)$ & $0.081(2)$ & $-0.005(2)$ & $0.0161(13)$ & $-0.0079(11)$ \\
\hline 07 & $0.053(2)$ & $0.048(2)$ & $0.071(2)$ & $0.0005(14)$ & $0.0259(14)$ & $-0.0145(12)$ \\
\hline 08 & $0.070(2)$ & $0.079(2)$ & $0.0416(14)$ & $0.0057(14)$ & $0.0214(13)$ & $-0.017(2)$ \\
\hline C1 & $0.039(2)$ & $0.035(2)$ & $0.044(2)$ & $-0.003(2)$ & $0.017(2)$ & $-0.0051(14)$ \\
\hline C2 & $0.048(2)$ & $0.047(2)$ & $0.046(2)$ & $0.009(2)$ & $0.014(2)$ & $-0.004(2)$ \\
\hline C3 & $0.052(2)$ & $0.040(2)$ & $0.062(2)$ & $0.006(2)$ & $0.017(2)$ & $0.000(2)$ \\
\hline C4 & $0.046(2)$ & $0.037(2)$ & $0.057(2)$ & $-0.008(2)$ & $0.022(2)$ & $-0.007(2)$ \\
\hline C5 & $0.063(3)$ & $0.047(2)$ & $0.083(3)$ & $-0.007(2)$ & $0.027(2)$ & $-0.004(2)$ \\
\hline C6 & $0.081(3)$ & $0.058(3)$ & $0.080(3)$ & $-0.023(2)$ & $0.032(3)$ & $-0.014(2)$ \\
\hline C7 & $0.083(3)$ & $0.072(3)$ & $0.064(3)$ & $-0.019(2)$ & $0.033(2)$ & $-0.017(3)$ \\
\hline C8 & $0.065(3)$ & $0.055(2)$ & $0.044(2)$ & $-0.013(2)$ & $0.015(2)$ & $-0.012(2)$ \\
\hline C9 & $0.043(2)$ & $0.043(2)$ & $0.048(2)$ & $-0.006(2)$ & $0.015(2)$ & $-0.008(2)$ \\
\hline C10 & $0.038(2)$ & $0.043(2)$ & $0.042(2)$ & $-0.001(2)$ & $0.011(2)$ & $-0.0092(14)$ \\
\hline C11 & $0.046(2)$ & $0.038(2)$ & $0.036(2)$ & $-0.001(2)$ & $0.014(2)$ & $-0.008(2)$ \\
\hline C12 & $0.041(2)$ & $0.048(2)$ & $0.040(2)$ & $0.003(2)$ & $0.008(2)$ & $-0.009(2)$ \\
\hline C13 & $0.048(2)$ & $0.050(2)$ & $0.052(2)$ & $0.001(2)$ & $0.009(2)$ & $-0.011(2)$ \\
\hline C14 & $0.048(3)$ & $0.066(3)$ & $0.122(5)$ & $0.015(3)$ & $-0.006(3)$ & $-0.015(2)$ \\
\hline C15 & $0.066(3)$ & $0.100(4)$ & $0.125(5)$ & $0.040(4)$ & $-0.027(3)$ & $-0.030(3)$ \\
\hline C16 & $0.056(3)$ & $0.072(3)$ & $0.070(3)$ & $0.017(2)$ & $-0.002(2)$ & $-0.007(2)$ \\
\hline C17 & $0.050(2)$ & $0.047(2)$ & $0.049(2)$ & $0.008(2)$ & $0.008(2)$ & $-0.004(2)$ \\
\hline C18 & $0.061(3)$ & $0.047(2)$ & $0.062(3)$ & $0.016(2)$ & $0.005(2)$ & $-0.003(2)$ \\
\hline C19 & $0.064(3)$ & $0.047(2)$ & $0.054(2)$ & $0.007(2)$ & $0.010(2)$ & $-0.018(2)$ \\
\hline C20 & $0.049(2)$ & $0.044(2)$ & $0.039(2)$ & $0.000(2)$ & $0.015(2)$ & $-0.009(2)$ \\
\hline C21 & $0.053(2)$ & $0.055(2)$ & $0.052(2)$ & $0.006(2)$ & $0.015(2)$ & $-0.017(2)$ \\
\hline C22 & $0.057(3)$ & $0.090(4)$ & $0.066(3)$ & $0.017(3)$ & $0.014(2)$ & $-0.018(2)$ \\
\hline $\mathrm{C} 23$ & $0.105(4)$ & $0.059(3)$ & $0.137(5)$ & $0.029(3)$ & $0.058(4)$ & $-0.002(3)$ \\
\hline C24 & $0.052(3)$ & $0.208(8)$ & $0.060(3)$ & $0.039(4)$ & $-0.002(2)$ & $-0.022(4)$ \\
\hline C25 & $0.047(2)$ & $0.096(4)$ & $0.075(3)$ & $0.010(3)$ & $0.003(2)$ & $-0.011(2)$ \\
\hline C26 & $0.077(4)$ & $0.200(8)$ & $0.082(4)$ & $0.061(5)$ & $0.026(3)$ & $0.017(4)$ \\
\hline C27 & $0.047(2)$ & $0.043(2)$ & $0.047(2)$ & $-0.006(2)$ & $0.018(2)$ & $-0.007(2)$ \\
\hline C28 & $0.063(3)$ & $0.049(2)$ & $0.066(3)$ & $0.003(2)$ & $0.020(2)$ & $-0.003(2)$ \\
\hline C29 & $0.059(3)$ & $0.069(3)$ & $0.055(2)$ & $-0.015(2)$ & $0.012(2)$ & $-0.020(2)$ \\
\hline C30 & $0.038(2)$ & $0.059(3)$ & $0.071(3)$ & $-0.003(2)$ & $0.017(2)$ & $-0.007(2)$ \\
\hline C31 & $0.078(4)$ & $0.109(5)$ & $0.076(3)$ & $-0.014(3)$ & $0.003(3)$ & $0.001(3)$ \\
\hline C32 & $0.050(3)$ & $0.114(5)$ & $0.105(4)$ & $-0.044(4)$ & $0.018(3)$ & $0.009(3)$ \\
\hline C33 & $0.060(2)$ & $0.047(2)$ & $0.063(2)$ & $0.002(2)$ & $0.020(2)$ & $-0.015(2)$ \\
\hline C34 & $0.071(3)$ & $0.067(3)$ & $0.087(3)$ & $0.025(3)$ & $0.011(3)$ & $-0.012(2)$ \\
\hline C35 & $0.059(3)$ & $0.076(3)$ & $0.076(3)$ & $0.008(3)$ & $0.007(2)$ & $-0.030(2)$ \\
\hline C36 & $0.111(4)$ & $0.089(4)$ & $0.045(2)$ & $0.007(2)$ & $0.031(3)$ & $-0.025(3)$ \\
\hline C37 & $0.124(5)$ & $0.116(5)$ & $0.044(3)$ & $-0.003(3)$ & $0.009(3)$ & $-0.026(4)$ \\
\hline C38 & $0.121(5)$ & $0.190(8)$ & $0.078(4)$ & $-0.029(4)$ & $0.060(4)$ & $-0.071(5)$ \\
\hline
\end{tabular}

Table S14. Bond Distances in $\mathbf{8}, \AA$ 


\begin{tabular}{|llllll|}
\hline Ti1-O4 & $1.773(3)$ & Ti1-O3 & $1.802(3)$ & Ti1-O2 & $1.862(3)$ \\
Ti1-O5 & $1.931(2)$ & Ti1-O1 & $2.117(2)$ & Ti1-Ti2 & $3.3156(8)$ \\
Ti2-O7 & $1.763(3)$ & Ti2-O8 & $1.784(3)$ & Ti2-O6 & $1.790(3)$ \\
Ti2-O1 & $1.988(2)$ & Ti2-O5 & $2.110(2)$ & O1-C1 & $1.380(4)$ \\
O2-C20 & $1.366(4)$ & O3-C21 & $1.414(4)$ & O4-C24 & $1.387(6)$ \\
O5-C27 & $1.451(4)$ & O6-C30 & $1.419(4)$ & O7-C33 & $1.412(5)$ \\
O8-C36 & $1.420(5)$ & C1-C2 & $1.387(5)$ & C1-C10 & $1.392(5)$ \\
C2-C3 & $1.388(5)$ & C3-C4 & $1.387(6)$ & C4-C9 & $1.397(5)$ \\
C4-C5 & $1.514(6)$ & C5-C6 & $1.513(7)$ & C6-C7 & $1.490(7)$ \\
C7-C8 & $1.520(6)$ & C8-C9 & $1.514(5)$ & C9-C10 & $1.417(5)$ \\
C10-C11 & $1.495(5)$ & C11-C20 & $1.400(5)$ & C11-C12 & $1.410(5)$ \\
C12-C17 & $1.398(5)$ & C12-C13 & $1.507(5)$ & C13-C14 & $1.481(6)$ \\
C14-C15 & $1.431(7)$ & C15-C16 & $1.516(7)$ & C16-C17 & $1.520(6)$ \\
C17-C18 & $1.395(6)$ & C18-C19 & $1.381(6)$ & C19-C20 & $1.398(5)$ \\
C21-C22 & $1.496(6)$ & C21-C23 & $1.501(6)$ & C24-C26 & $1.420(6)$ \\
C24-C25 & $1.445(7)$ & C27-C29 & $1.502(5)$ & C27-C28 & $1.510(5)$ \\
C30-C31 & $1.492(7)$ & C30-C32 & $1.506(6)$ & C33-C34 & $1.491(6)$ \\
C33-C35 & $1.507(6)$ & C36-C38 & $1.460(8)$ & C36-C37 & $1.485(7)$ \\
\hline
\end{tabular}


Table S15. Bond Angles in 8,

\begin{tabular}{|c|c|c|c|c|c|}
\hline O4-Ti1-O3 & $103.35(13)$ & O4-Ti1-O2 & $109.38(12)$ & O3-Ti1-O2 & $94.39(11)$ \\
\hline O4-Ti1-O5 & $112.13(11)$ & O3-Ti1-O5 & $96.20(11)$ & O2-Ti1-O5 & $133.20(11)$ \\
\hline O4-Ti1-O1 & $95.20(11)$ & O3-Ti1-O1 & $160.69(12)$ & O2-Ti1-O1 & $84.29(10)$ \\
\hline O5-Ti1-O1 & $71.50(10)$ & O4-Ti1-Ti2 & $105.72(9)$ & O3-Ti1-Ti2 & $131.54(9)$ \\
\hline O2-Ti1-Ti2 & $111.02(8)$ & O5-Ti1-Ti2 & $36.68(7)$ & O1-Ti1-Ti2 & $34.83(6)$ \\
\hline O7-Ti2-O8 & $102.83(14)$ & O7-Ti2-O6 & $99.05(13)$ & O8-Ti2-06 & $111.6(2)$ \\
\hline O7-Ti2-O1 & $92.02(11)$ & O8-Ti2-O1 & $117.13(12)$ & O6-Ti2-01 & 125.93(12) \\
\hline O7-Ti2-O5 & $161.42(11)$ & O8-Ti2-O5 & $91.32(12)$ & O6-Ti2-O5 & $86.60(11)$ \\
\hline O1-Ti2-O5 & $70.60(9)$ & O7-Ti2-Ti1 & 129.29(9) & O8-Ti2-Ti1 & $105.48(9)$ \\
\hline O6-Ti2-Ti1 & $108.20(9)$ & O1-Ti2-Ti1 & $37.48(7)$ & O5-Ti2-Ti1 & $33.14(6)$ \\
\hline C1-O1-Ti2 & $130.5(2)$ & C1-O1-Ti1 & $121.7(2)$ & Ti2-O1-Ti1 & $107.70(11)$ \\
\hline C20-O2-Ti1 & $118.9(2)$ & C21-O3-Ti1 & $138.7(3)$ & C24-O4-Ti1 & $145.2(4)$ \\
\hline C27-O5-Ti1 & $133.3(2)$ & C27-O5-Ti2 & $116.5(2)$ & Ti1-O5-Ti2 & $110.18(11)$ \\
\hline С30-O6-Ti2 & 140.1(3) & С33-O7-Тi2 & 168.3(3) & С36-O8-Тi2 & $147.7(3)$ \\
\hline $\mathrm{O} 1-\mathrm{C} 1-\mathrm{C} 2$ & $119.4(3)$ & $\mathrm{O} 1-\mathrm{C} 1-\mathrm{C} 10$ & $119.2(3)$ & $\mathrm{C} 2-\mathrm{C} 1-\mathrm{C} 10$ & 121.3(3) \\
\hline $\mathrm{C} 1-\mathrm{C} 2-\mathrm{C} 3$ & $118.3(4)$ & C4-C3-C2 & $122.2(4)$ & C3-C4-C9 & 119.1(4) \\
\hline C3-C4-C5 & $119.0(4)$ & C9-C4-C5 & $121.9(4)$ & C6-C5-C4 & $114.1(4)$ \\
\hline C7-C6-C5 & $110.1(4)$ & $\mathrm{C} 6-\mathrm{C} 7-\mathrm{C} 8$ & $112.1(4)$ & $\mathrm{C} 9-\mathrm{C} 8-\mathrm{C} 7$ & $112.9(4)$ \\
\hline C4-C9-C10 & $119.5(3)$ & C4-C9-C8 & $120.6(4)$ & C10-C9-C8 & 119.8(3) \\
\hline C1-C10-C9 & 119.2(3) & C1-C10-C11 & 118.2(3) & C9-C10-C11 & $122.5(3)$ \\
\hline C20-C11-C12 & $119.5(3)$ & C20-C11-C10 & $117.8(3)$ & C12-C11-C10 & $122.6(3)$ \\
\hline C17-C12-C11 & $119.6(3)$ & $\mathrm{C} 17-\mathrm{C} 12-\mathrm{C} 13$ & 119.6(3) & $\mathrm{C}_{11}-\mathrm{C}_{12}-\mathrm{C}_{13}$ & $120.7(3)$ \\
\hline C14-C13-C12 & $115.4(3)$ & C15-C14-C13 & $115.8(4)$ & C14-C15-C16 & $113.6(5)$ \\
\hline C15-C16-C17 & $112.8(4)$ & C18-C17-C12 & 119.6(4) & C18-C17-C16 & 118.0(3) \\
\hline C12-C17-C16 & $122.4(4)$ & C19-C18-C17 & $121.5(4)$ & C18-C19-C20 & $119.2(4)$ \\
\hline O2-C20-C19 & $119.6(3)$ & O2-C20-C11 & 119.7(3) & C19-C20-C11 & $120.6(3)$ \\
\hline O3-C21-C22 & 109.7(3) & O3-C21-C23 & $110.3(3)$ & C22-C21-C23 & $112.8(4)$ \\
\hline O4-C24-C26 & $115.2(5)$ & O4-C24-C25 & $114.1(4)$ & C26-C24-C25 & $121.4(5)$ \\
\hline O5-C27-C29 & $110.0(3)$ & O5-C27-C28 & $109.6(3)$ & C29-C27-C28 & $113.5(3)$ \\
\hline O6-C30-C31 & $109.9(4)$ & O6-С30-С32 & $108.5(4)$ & C31-C30-C32 & $112.5(4)$ \\
\hline О7-С33-С34 & $109.8(4)$ & О7-С33-С35 & 109.4(3) & C34-С33-С35 & $112.2(4)$ \\
\hline О8-С36-С38 & $111.5(5)$ & О8-С36-С37 & $109.4(4)$ & С38-С36-C37 & $114.4(5)$ \\
\hline
\end{tabular}


Ortep of 9 with full atom labeling
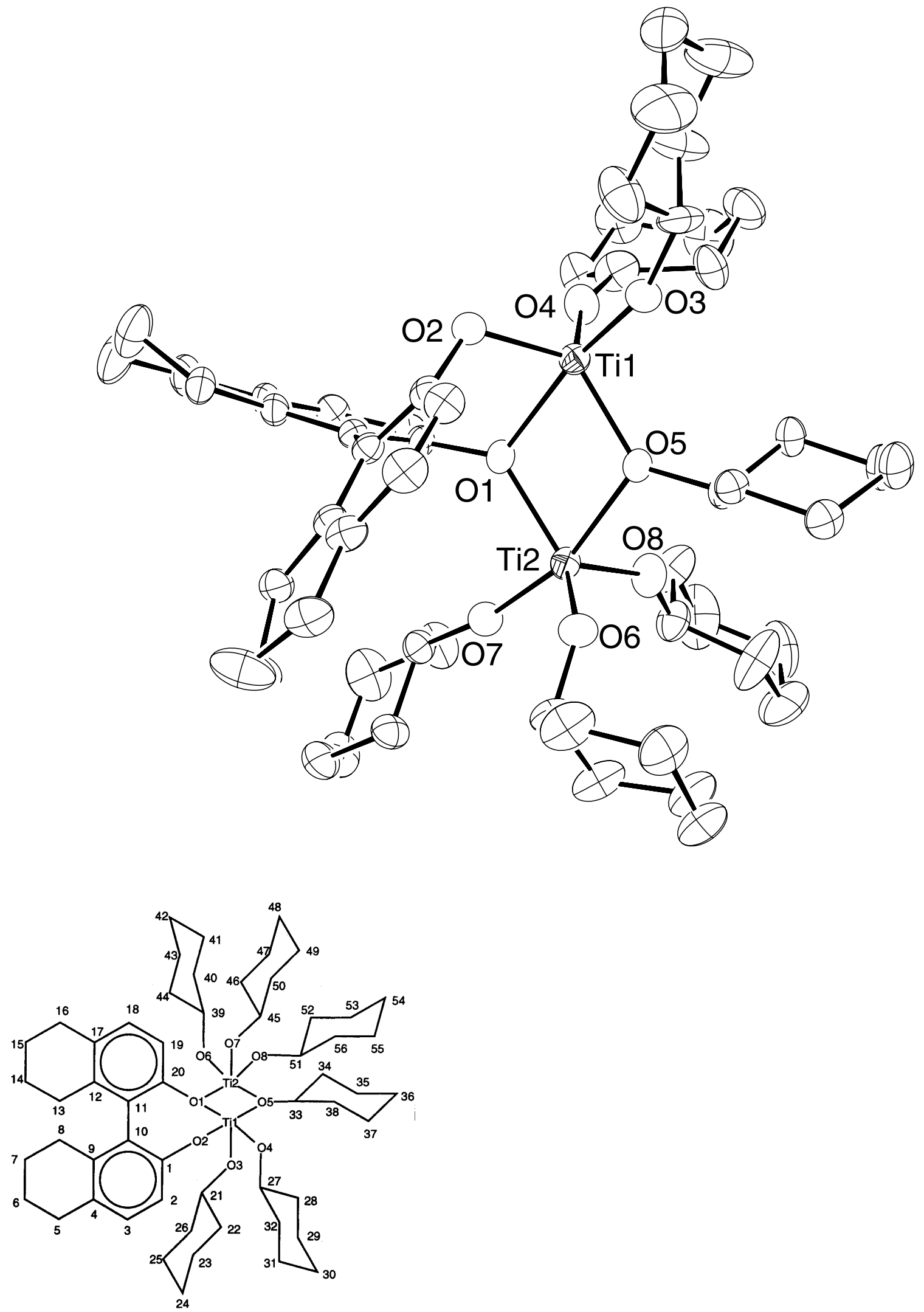
Table S16. Summary of Structure Determination of 9

Formula:

Formula weight:

Crystal class:

Space group:

$\mathrm{Z}$

Cell constants:

$\begin{array}{ll} & \mathrm{a} \\ & \mathrm{b} \\ & \mathrm{c} \\ & \beta \\ & \mathrm{V} \\ \mu & \\ \text { crystal size, } \mathrm{mm} \\ \mathrm{D}_{\text {calc }}\end{array}$

$\mathrm{F}(000)$

Radiation:

$2 \theta$ range

hkl collected:

No. reflections measured:

No. unique reflections:

No. observed reflections

No. reflections used in refinement

No. parameters

$\mathrm{R}$ indices $(\mathrm{F}>4 \sigma)$

$\mathrm{R}$ indices (all data)

GOF:

Final Difference Peaks, e/ $\AA^{3}$
$\mathrm{Ti}_{2} \mathrm{C}_{62} \mathrm{H}_{108} \mathrm{O}_{11}$

1125.28

monoclinic

$\mathrm{P} 2$ (\#4)

2

$14.00410(10) \AA$

$15.2164(2) \AA$

$15.6346(2) \AA$

$90.6120(10)^{\circ}$

$3331.42(7) \AA^{3}$

$2.92 \mathrm{~cm}^{-1}$

$0.34 \times 0.28 \times 0.26$

$1.122 \mathrm{~g} / \mathrm{cm}^{3}$

1224

$\operatorname{Mo}-K_{\alpha}(\lambda=0.71069 \AA)$

$5.22-50.7^{\circ}$

$-16 \leq \mathrm{h} \leq 16 ;-18 \leq \mathrm{k} \leq 18 ;-18 \leq 1 \leq 18$

26439

$11716\left(\mathrm{R}_{\mathrm{int}}=0.0326\right)$

$10098(\mathrm{~F}>4 \sigma)$

11716

757

$\mathrm{R}_{1}=0.0731$

$\mathrm{wR}_{2}=0.1914$

$\mathrm{R}_{1}=0.0830$

$\mathrm{wR}_{2}=0.2025$

1.102

$+0.315,-0.256$ 
Table S17. Refined Positional Parameters for 9

\begin{tabular}{|c|c|c|c|c|}
\hline Atom & $\mathrm{x}$ & $\mathrm{y}$ & $\mathrm{Z}$ & $\mathrm{U}_{\mathrm{eq}}, \AA^{2}$ \\
\hline Ti1 & $0.84207(6)$ & $0.45361(6)$ & $0.30406(5)$ & $0.0594(2)$ \\
\hline Ti2 & $0.68894(6)$ & $0.59038(6)$ & $0.20970(6)$ & $0.0589(2)$ \\
\hline 01 & $0.6992(2)$ & $0.4704(2)$ & $0.2573(2)$ & $0.0588(8)$ \\
\hline $\mathrm{O} 2$ & $0.8354(3)$ & $0.3450(2)$ & $0.2464(2)$ & $0.0667(9)$ \\
\hline $\mathrm{O} 3$ & $0.9680(3)$ & $0.4536(3)$ & $0.3183(3)$ & $0.0801(10)$ \\
\hline O4 & $0.8021(3)$ & 0.4394(3) & $0.4091(2)$ & $0.0885(12)$ \\
\hline O5 & $0.8296(2)$ & $0.5701(2)$ & $0.2576(2)$ & $0.0609(8)$ \\
\hline O6 & $0.7389(3)$ & $0.6232(3)$ & $0.1090(3)$ & $0.0777(10)$ \\
\hline $\mathrm{O} 7$ & $0.5687(2)$ & $0.5747(3)$ & $0.1775(3)$ & $0.0754(10)$ \\
\hline O8 & $0.6776(3)$ & $0.6804(3)$ & $0.2821(3)$ & $0.0960(14)$ \\
\hline C1 & $0.6343(3)$ & $0.4020(3)$ & $0.2565(3)$ & $0.0561(10)$ \\
\hline C2 & $0.5626(4)$ & $0.4005(4)$ & $0.3188(3)$ & $0.0654(12)$ \\
\hline $\mathrm{H} 2$ & $0.5559(4)$ & $0.4458(4)$ & $0.3583(3)$ & 0.087 \\
\hline C3 & $0.5022(4)$ & $0.3278(4)$ & $0.3184(3)$ & $0.0748(14)$ \\
\hline $\mathrm{H} 3$ & $0.4528(4)$ & $0.3260(4)$ & $0.3576(3)$ & 0.099 \\
\hline C4 & $0.5122(4)$ & $0.2590(4)$ & $0.2632(3)$ & $0.0700(13)$ \\
\hline C5 & $0.4462(6)$ & $0.1806(5)$ & $0.2700(4)$ & $0.103(2)$ \\
\hline $\mathrm{H} 5 \mathrm{a}$ & $0.4486(6)$ & $0.1592(5)$ & $0.3284(4)$ & 0.137 \\
\hline $\mathrm{H} 5 \mathrm{~b}$ & $0.3814(6)$ & $0.2002(5)$ & $0.2584(4)$ & 0.137 \\
\hline C6 & $0.4679(7)$ & $0.1069(6)$ & $0.2121(8)$ & $0.146(4)$ \\
\hline $\mathrm{H} 6 \mathrm{a}$ & $0.4528(7)$ & $0.0530(6)$ & $0.2420(8)$ & 0.194 \\
\hline $\mathrm{H} 6 \mathrm{~b}$ & $0.4244(7)$ & $0.1110(6)$ & $0.1635(8)$ & 0.194 \\
\hline C7 & $0.5635(8)$ & $0.0979(5)$ & $0.1788(6)$ & $0.131(3)$ \\
\hline $\mathrm{H} 7 \mathrm{a}$ & $0.5622(8)$ & $0.0553(5)$ & $0.1326(6)$ & 0.174 \\
\hline $\mathrm{H} 7 \mathrm{~b}$ & $0.6048(8)$ & $0.0748(5)$ & $0.2236(6)$ & 0.174 \\
\hline C8 & $0.6057(5)$ & $0.1822(4)$ & $0.1461(4)$ & $0.081(2)$ \\
\hline $\mathrm{H} 8 \mathrm{a}$ & $0.5811(5)$ & $0.1930(4)$ & $0.0889(4)$ & 0.108 \\
\hline $\mathrm{H} 8 \mathrm{~b}$ & $0.6743(5)$ & $0.1752(4)$ & $0.1422(4)$ & 0.108 \\
\hline C9 & $0.5844(4)$ & $0.2622(3)$ & $0.2018(3)$ & $0.0633(12)$ \\
\hline C10 & $0.6434(3)$ & $0.3356(3)$ & $0.1969(3)$ & $0.0539(10)$ \\
\hline C11 & $0.7201(3)$ & $0.3439(3)$ & $0.1321(3)$ & $0.0566(10)$ \\
\hline C12 & $0.6989(4)$ & $0.3548(3)$ & $0.0442(3)$ & $0.0609(11)$ \\
\hline C13 & $0.5978(4)$ & $0.3618(4)$ & $0.0138(3)$ & $0.0693(13)$ \\
\hline $\mathrm{H} 13 \mathrm{a}$ & $0.5682(4)$ & $0.3044(4)$ & $0.0182(3)$ & 0.092 \\
\hline $\mathrm{H} 13 \mathrm{~b}$ & $0.5639(4)$ & $0.4011(4)$ & $0.0519(3)$ & 0.092 \\
\hline C14 & $0.5851(5)$ & $0.3942(6)$ & $-0.0755(4)$ & $0.101(2)$ \\
\hline $\mathrm{H} 14 \mathrm{a}$ & $0.5234(5)$ & $0.3744(6)$ & $-0.0966(4)$ & 0.134 \\
\hline $\mathrm{H} 14 \mathrm{~b}$ & $0.5837(5)$ & $0.4579(6)$ & $-0.0742(4)$ & 0.134 \\
\hline C15 & $0.6535(7)$ & $0.3684(9)$ & $-0.1327(5)$ & $0.144(4)$ \\
\hline $\mathrm{H} 15 \mathrm{a}$ & $0.6433(7)$ & $0.4017(9)$ & $-0.1849(5)$ & 0.191 \\
\hline $\mathrm{H} 15 \mathrm{~b}$ & $0.6418(7)$ & $0.3071(9)$ & $-0.1464(5)$ & 0.191 \\
\hline C16 & $0.7563(6)$ & $0.3771(5)$ & $-0.1083(4)$ & $0.095(2)$ \\
\hline $\mathrm{H} 16 \mathrm{a}$ & $0.7782(6)$ & $0.4355(5)$ & $-0.1234(4)$ & 0.127 \\
\hline $\mathrm{H} 16 \mathrm{~b}$ & $0.7932(6)$ & $0.3351(5)$ & $-0.1408(4)$ & 0.127 \\
\hline C17 & $0.7745(5)$ & $0.3621(4)$ & $-0.0139(3)$ & $0.0748(14)$ \\
\hline C18 & $0.8696(5)$ & $0.3599(5)$ & $0.0178(4)$ & $0.087(2)$ \\
\hline $\mathrm{H} 18$ & $0.9199(5)$ & $0.3626(5)$ & $-0.0205(4)$ & 0.116 \\
\hline C19 & $0.8886(4)$ & $0.3541(4)$ & $0.1008(4)$ & $0.077(2)$ \\
\hline $\mathrm{H} 19$ & $0.9516(4)$ & $0.3547(4)$ & $0.1200(4)$ & 0.102 \\
\hline C20 & $0.8149(4)$ & $0.3471(3)$ & $0.1593(3)$ & $0.0658(12)$ \\
\hline C22 & $1.0549(5)$ & $0.3855(7)$ & $0.4344(4)$ & $0.137(4)$ \\
\hline $\mathrm{H} 22 \mathrm{a}$ & $1.0112(5)$ & $0.3361(7)$ & $0.4341(4)$ & 0.182 \\
\hline $\mathrm{H} 22 \mathrm{~b}$ & $1.0314(5)$ & $0.4289(7)$ & $0.4744(4)$ & 0.182 \\
\hline
\end{tabular}




\begin{tabular}{|c|c|c|c|c|}
\hline $\mathrm{H}^{2} 2 \mathrm{a}^{\prime}$ & $1.0810(5)$ & $0.4426(7)$ & $0.4492(4)$ & 0.182 \\
\hline $\mathrm{H} 22 \mathrm{~b}^{\prime}$ & $0.9972(5)$ & $0.3766(7)$ & $0.4671(4)$ & 0.182 \\
\hline C25 & $1.1959(6)$ & $0.3254(8)$ & $0.3087(6)$ & $0.170(5)$ \\
\hline $\mathrm{H} 25 \mathrm{a}$ & $1.2195(6)$ & $0.2820(8)$ & $0.2688(6)$ & 0.225 \\
\hline $\mathrm{H} 25 \mathrm{~b}$ & $1.2388(6)$ & $0.3755(8)$ & $0.3082(6)$ & 0.225 \\
\hline H25a' & $1.1697(6)$ & $0.2684(8)$ & $0.2940(6)$ & 0.225 \\
\hline H25b' & $1.2536(6)$ & $0.3342(8)$ & $0.2760(6)$ & 0.225 \\
\hline C21 & $1.0598(7)$ & $0.4255(7)$ & $0.3449(6)$ & $0.092(4)$ \\
\hline H21 & $1.1040(7)$ & $0.4754(7)$ & $0.3452(6)$ & 0.123 \\
\hline C23 & $1.1556(9)$ & $0.3546(12)$ & $0.4626(6)$ & $0.172(11)$ \\
\hline $\mathrm{H} 23 \mathrm{a}$ & $1.1989(9)$ & $0.4044(12)$ & $0.4644(6)$ & 0.228 \\
\hline $\mathrm{H} 23 \mathrm{~b}$ & $1.1530(9)$ & $0.3293(12)$ & $0.5195(6)$ & 0.228 \\
\hline C24 & $1.1926(10)$ & $0.2859(9)$ & $0.3994(8)$ & $0.169(11)$ \\
\hline $\mathrm{H} 24 \mathrm{a}$ & $1.1509(10)$ & $0.2350(9)$ & $0.3994(8)$ & 0.225 \\
\hline $\mathrm{H} 24 \mathrm{~b}$ & $1.2560(10)$ & $0.2669(9)$ & $0.4168(8)$ & 0.225 \\
\hline C26 & $1.0945(8)$ & $0.3547(11)$ & $0.2817(6)$ & $0.133(7)$ \\
\hline $\mathrm{H} 26 \mathrm{a}$ & $1.0515(8)$ & $0.3047(11)$ & $0.2823(6)$ & 0.177 \\
\hline $\mathrm{H} 26 \mathrm{~b}$ & $1.0954(8)$ & $0.3784(11)$ & $0.2241(6)$ & 0.177 \\
\hline C21' & $1.0312(7)$ & $0.3821(13)$ & $0.3375(7)$ & $0.115(9)$ \\
\hline $\mathrm{H} 21^{\prime}$ & $1.0019(7)$ & $0.3257(13)$ & $0.3219(7)$ & 0.152 \\
\hline C23' & $1.1281(12)$ & $0.313(2)$ & $0.4561(9)$ & $0.27(3)$ \\
\hline H23a' & $1.1015(12)$ & $0.256(2)$ & $0.4420(9)$ & 0.358 \\
\hline H23b' & $1.1428(12)$ & $0.314(2)$ & $0.5168(9)$ & 0.358 \\
\hline C24' & $1.2199(8)$ & $0.3285(14)$ & $0.4048(8)$ & $0.135(11)$ \\
\hline H24a' & $1.2471(8)$ & $0.3852(14)$ & $0.4194(8)$ & 0.179 \\
\hline H24b' & $1.2665(8)$ & $0.2835(14)$ & $0.4188(8)$ & 0.179 \\
\hline C26' & $1.1227(8)$ & $0.3979(10)$ & $0.2865(7)$ & $0.103(7)$ \\
\hline H26a' & $1.1491(8)$ & $0.4551(10)$ & $0.3007(7)$ & 0.137 \\
\hline H26b' & $1.1083(8)$ & $0.3969(10)$ & $0.2257(7)$ & 0.137 \\
\hline C27 & $0.7933(5)$ & $0.4556(5)$ & $0.4914(3)$ & $0.107(2)$ \\
\hline $\mathrm{H} 27$ & $0.8276(5)$ & $0.4044(5)$ & $0.5141(3)$ & 0.142 \\
\hline H27' & $0.8541(5)$ & $0.4874(5)$ & $0.4920(3)$ & 0.142 \\
\hline C30 & $0.7622(7)$ & $0.5332(6)$ & $0.6597(4)$ & $0.160(4)$ \\
\hline $\mathrm{H} 30 \mathrm{a}$ & $0.7633(7)$ & $0.5401(6)$ & $0.7214(4)$ & 0.213 \\
\hline $\mathrm{H} 30 \mathrm{~b}$ & $0.7290(7)$ & $0.5829(6)$ & $0.6345(4)$ & 0.213 \\
\hline H30a' & $0.8242(7)$ & $0.5616(6)$ & $0.6611(4)$ & 0.213 \\
\hline H30b' & $0.7297(7)$ & $0.5466(6)$ & $0.7126(4)$ & 0.213 \\
\hline C28 & $0.8589(7)$ & $0.5278(8)$ & $0.5260(5)$ & $0.103(4)$ \\
\hline $\mathrm{H} 28 \mathrm{a}$ & $0.8344(7)$ & $0.5846(8)$ & $0.5079(5)$ & 0.137 \\
\hline $\mathrm{H} 28 \mathrm{~b}$ & $0.9219(7)$ & $0.5207(8)$ & $0.5018(5)$ & 0.137 \\
\hline C29 & $0.8671(7)$ & $0.5261(9)$ & $0.6241(6)$ & $0.126(5)$ \\
\hline $\mathrm{H} 29 \mathrm{a}$ & $0.9056(7)$ & $0.5750(9)$ & $0.6443(6)$ & 0.168 \\
\hline $\mathrm{H} 29 \mathrm{~b}$ & $0.8968(7)$ & $0.4718(9)$ & $0.6430(6)$ & 0.168 \\
\hline C31 & $0.7125(10)$ & $0.4458(10)$ & $0.6340(5)$ & $0.140(6)$ \\
\hline H31a & $0.7506(10)$ & $0.3962(10)$ & $0.6533(5)$ & 0.186 \\
\hline $\mathrm{H} 31 \mathrm{~b}$ & $0.6503(10)$ & $0.4420(10)$ & $0.6604(5)$ & 0.186 \\
\hline C32 & $0.7018(6)$ & $0.4440(10)$ & $0.5369(6)$ & $0.123(5)$ \\
\hline H32a & $0.6736(6)$ & $0.3883(10)$ & $0.5199(6)$ & 0.163 \\
\hline H32b & $0.6581(6)$ & $0.4903(10)$ & $0.5194(6)$ & 0.163 \\
\hline C28' & $0.7390(11)$ & $0.5410(8)$ & $0.4966(5)$ & $0.085(5)$ \\
\hline H28a' & $0.7805(11)$ & $0.5875(8)$ & $0.4763(5)$ & 0.113 \\
\hline H28b' & $0.6852(11)$ & $0.5376(8)$ & $0.4572(5)$ & 0.113 \\
\hline C29' & $0.702(2)$ & $0.5679(11)$ & $0.5817(7)$ & $0.19(2)$ \\
\hline H29a' & $0.637(2)$ & $0.5468(11)$ & $0.5869(7)$ & 0.257 \\
\hline H29b' & $0.700(2)$ & $0.6316(11)$ & $0.5842(7)$ & 0.257 \\
\hline C31' & $0.775(2)$ & $0.4347(8)$ & $0.6509(5)$ & $0.113(8)$ \\
\hline H31a' & $0.814(2)$ & $0.4133(8)$ & $0.6981(5)$ & 0.151 \\
\hline H31b' & $0.712(2)$ & $0.4066(8)$ & $0.6546(5)$ & 0.151 \\
\hline
\end{tabular}




\begin{tabular}{|c|c|c|c|c|}
\hline C32' & $0.821(2)$ & $0.4091(9)$ & $0.5675(5)$ & $0.106(6)$ \\
\hline H32a' & $0.889(2)$ & $0.4154(9)$ & $0.5748(5)$ & 0.141 \\
\hline H32b' & $0.808(2)$ & $0.3472(9)$ & $0.5579(5)$ & 0.141 \\
\hline C33 & $0.8936(3)$ & $0.6450(3)$ & $0.2502(3)$ & $0.0640(12)$ \\
\hline H33 & $0.8565(3)$ & $0.6946(3)$ & $0.2275(3)$ & 0.085 \\
\hline C34 & $0.9326(4)$ & $0.6711(4)$ & $0.3367(3)$ & $0.0718(13)$ \\
\hline H34a & $0.9705(4)$ & $0.6234(4)$ & $0.3602(3)$ & 0.095 \\
\hline $\mathrm{H} 34 \mathrm{~b}$ & $0.8802(4)$ & $0.6824(4)$ & $0.3752(3)$ & 0.095 \\
\hline C35 & $0.9941(5)$ & $0.7529(5)$ & $0.3291(4)$ & $0.089(2)$ \\
\hline H35a & $1.0212(5)$ & $0.7676(5)$ & $0.3846(4)$ & 0.118 \\
\hline H35b & $0.9550(5)$ & $0.8019(5)$ & $0.3100(4)$ & 0.118 \\
\hline C36 & $1.0749(5)$ & $0.7370(5)$ & $0.2648(5)$ & $0.098(2)$ \\
\hline $\mathrm{H} 36 \mathrm{a}$ & $1.1107(5)$ & $0.7910(5)$ & $0.2573(5)$ & 0.131 \\
\hline $\mathrm{H} 36 \mathrm{~b}$ & $1.1182(5)$ & $0.6929(5)$ & $0.2875(5)$ & 0.131 \\
\hline C37 & $1.0360(4)$ & $0.7067(4)$ & $0.1787(4)$ & $0.078(2)$ \\
\hline H37a & $0.9987(4)$ & $0.7536(4)$ & $0.1528(4)$ & 0.104 \\
\hline H37b & $1.0887(4)$ & $0.6936(4)$ & $0.1411(4)$ & 0.104 \\
\hline C38 & $0.9743(3)$ & $0.6262(3)$ & $0.1880(3)$ & $0.0630(12)$ \\
\hline H38a & $1.0127(3)$ & $0.5776(3)$ & $0.2091(3)$ & 0.084 \\
\hline H38b & $0.9478(3)$ & $0.6098(3)$ & $0.1327(3)$ & 0.084 \\
\hline C39 & $0.7051(4)$ & $0.6510(3)$ & $0.0275(4)$ & $0.086(2)$ \\
\hline H39 & $0.6561(4)$ & $0.6093(3)$ & $0.0081(4)$ & 0.114 \\
\hline C40 & $0.7868(5)$ & $0.6465(4)$ & $-0.0337(5)$ & $0.122(3)$ \\
\hline $\mathrm{H} 40 \mathrm{a}$ & $0.8241(5)$ & $0.5943(4)$ & $-0.0209(5)$ & 0.163 \\
\hline $\mathrm{H} 40 \mathrm{~b}$ & $0.7611(5)$ & $0.6402(4)$ & $-0.0912(5)$ & 0.163 \\
\hline H40a' & $0.8441(5)$ & $0.6660(4)$ & $-0.0038(5)$ & 0.163 \\
\hline H40b' & $0.7963(5)$ & $0.5855(4)$ & $-0.0493(5)$ & 0.163 \\
\hline C44 & $0.6586(5)$ & $0.7405(4)$ & $0.0362(5)$ & $0.118(3)$ \\
\hline $\mathrm{H} 44 \mathrm{a}$ & $0.6191(5)$ & $0.7516(4)$ & $-0.0139(5)$ & 0.158 \\
\hline $\mathrm{H} 44 \mathrm{~b}$ & $0.6173(5)$ & $0.7400(4)$ & $0.0856(5)$ & 0.158 \\
\hline $\mathrm{H} 44 \mathrm{a}^{\prime}$ & $0.5960(5)$ & $0.7329(4)$ & $0.0610(5)$ & 0.158 \\
\hline H44b' & $0.6965(5)$ & $0.7757(4)$ & $0.0757(5)$ & 0.158 \\
\hline C41 & $0.8520(6)$ & $0.7246(6)$ & $-0.0316(9)$ & $0.121(5)$ \\
\hline $\mathrm{H} 41 \mathrm{a}$ & $0.8928(6)$ & $0.7227(6)$ & $-0.0812(9)$ & 0.161 \\
\hline $\mathrm{H} 41 \mathrm{~b}$ & $0.8926(6)$ & $0.7209(6)$ & $0.0189(9)$ & 0.161 \\
\hline C42 & $0.7991(9)$ & $0.8126(5)$ & $-0.0307(9)$ & $0.128(5)$ \\
\hline $\mathrm{H} 42 \mathrm{a}$ & $0.7636(9)$ & $0.8203(5)$ & $-0.0838(9)$ & 0.170 \\
\hline $\mathrm{H} 42 \mathrm{~b}$ & $0.8445(9)$ & $0.8605(5)$ & $-0.0252(9)$ & 0.170 \\
\hline C43 & $0.7294(8)$ & $0.8136(5)$ & $0.0460(8)$ & $0.120(5)$ \\
\hline $\mathrm{H} 43 \mathrm{a}$ & $0.7651(8)$ & $0.8065(5)$ & $0.0991(8)$ & 0.160 \\
\hline $\mathrm{H} 43 \mathrm{~b}$ & $0.6963(8)$ & $0.8695(5)$ & $0.0480(8)$ & 0.160 \\
\hline C41' & $0.7767(12)$ & $0.6987(10)$ & $-0.1136(7)$ & $0.115(7)$ \\
\hline H41a' & $0.7324(12)$ & $0.6691(10)$ & $-0.1520(7)$ & 0.154 \\
\hline H41b' & $0.8381(12)$ & $0.7016(10)$ & $-0.1415(7)$ & 0.154 \\
\hline C42' & $0.7409(14)$ & $0.7924(9)$ & $-0.0972(10)$ & $0.133(9)$ \\
\hline H42a' & $0.7298(14)$ & $0.8221(9)$ & $-0.1513(10)$ & 0.176 \\
\hline H42b' & $0.7890(14)$ & $0.8252(9)$ & $-0.0654(10)$ & 0.176 \\
\hline C43' & $0.6479(12)$ & $0.7898(10)$ & $-0.0462(9)$ & $0.126(8)$ \\
\hline H43a' & $0.6277(12)$ & $0.8495(10)$ & $-0.0341(9)$ & 0.167 \\
\hline H43b' & $0.5983(12)$ & $0.7623(10)$ & $-0.0808(9)$ & 0.167 \\
\hline C45 & $0.4739(4)$ & $0.5494(4)$ & $0.1635(4)$ & $0.0703(13)$ \\
\hline $\mathrm{H} 45$ & $0.4721(4)$ & $0.4850(4)$ & $0.1653(4)$ & 0.094 \\
\hline C46 & $0.4082(4)$ & $0.5823(6)$ & $0.2325(5)$ & $0.107(2)$ \\
\hline $\mathrm{H} 46 \mathrm{a}$ & $0.4113(4)$ & $0.6459(6)$ & $0.2349(5)$ & 0.142 \\
\hline $\mathrm{H} 46 \mathrm{~b}$ & $0.4293(4)$ & $0.5595(6)$ & $0.2874(5)$ & 0.142 \\
\hline C47 & $0.3055(5)$ & $0.5540(8)$ & $0.2156(7)$ & $0.137(3)$ \\
\hline $\mathrm{H} 47 \mathrm{a}$ & $0.3009(5)$ & $0.4907(8)$ & $0.2214(7)$ & 0.182 \\
\hline $\mathrm{H} 47 \mathrm{~b}$ & $0.2646(5)$ & $0.5804(8)$ & $0.2582(7)$ & 0.182 \\
\hline
\end{tabular}




\begin{tabular}{|c|c|c|c|c|}
\hline C48 & $0.2710(4)$ & $0.5798(6)$ & $0.1293(6)$ & $0.118(3)$ \\
\hline $\mathrm{H} 48 \mathrm{a}$ & $0.2083(4)$ & $0.5545(6)$ & $0.1192(6)$ & 0.156 \\
\hline $\mathrm{H} 48 \mathrm{~b}$ & $0.2648(4)$ & $0.6432(6)$ & $0.1267(6)$ & 0.156 \\
\hline C49 & $0.3362(5)$ & $0.5503(6)$ & $0.0617(5)$ & $0.106(2)$ \\
\hline $\mathrm{H} 49 \mathrm{a}$ & $0.3145(5)$ & $0.5744(6)$ & $0.0074(5)$ & 0.141 \\
\hline $\mathrm{H} 49 \mathrm{~b}$ & $0.3329(5)$ & $0.4868(6)$ & $0.0575(5)$ & 0.141 \\
\hline C50 & $0.4390(4)$ & $0.5768(5)$ & $0.0763(4)$ & $0.084(2)$ \\
\hline $\mathrm{H} 50 \mathrm{a}$ & $0.4785(4)$ & $0.5497(5)$ & $0.0331(4)$ & 0.111 \\
\hline $\mathrm{H} 50 \mathrm{~b}$ & $0.4447(4)$ & $0.6400(5)$ & $0.0707(4)$ & 0.111 \\
\hline C51 & $0.6164(5)$ & $0.7468(3)$ & $0.3065(4)$ & $0.090(2)$ \\
\hline H51 & $0.5612(5)$ & $0.7464(3)$ & $0.2674(4)$ & 0.119 \\
\hline C52 & $0.5803(7)$ & $0.7299(4)$ & $0.3969(6)$ & $0.139(3)$ \\
\hline $\mathrm{H} 52 \mathrm{a}$ & $0.6341(7)$ & $0.7281(4)$ & $0.4366(6)$ & 0.185 \\
\hline $\mathrm{H} 52 \mathrm{~b}$ & $0.5485(7)$ & $0.6733(4)$ & $0.3988(6)$ & 0.185 \\
\hline C53 & $0.5107(7)$ & $0.8021(6)$ & $0.4243(7)$ & $0.190(6)$ \\
\hline H53a & $0.4557(7)$ & $0.8028(6)$ & $0.3861(7)$ & 0.253 \\
\hline H53b & $0.4885(7)$ & $0.7905(6)$ & $0.4818(7)$ & 0.253 \\
\hline C54 & $0.5614(8)$ & $0.8912(6)$ & $0.4217(7)$ & $0.172(5)$ \\
\hline $\mathrm{H} 54 \mathrm{a}$ & $0.5171(8)$ & $0.9372(6)$ & $0.4378(7)$ & 0.228 \\
\hline $\mathrm{H} 54 \mathrm{~b}$ & $0.6140(8)$ & $0.8914(6)$ & $0.4626(7)$ & 0.228 \\
\hline C55 & $0.5992(9)$ & $0.9096(4)$ & $0.3324(7)$ & $0.190(6)$ \\
\hline $\mathrm{H} 55 \mathrm{a}$ & $0.5457(9)$ & $0.9163(4)$ & $0.2929(7)$ & 0.253 \\
\hline H55b & $0.6345(9)$ & $0.9644(4)$ & $0.3331(7)$ & 0.253 \\
\hline C56 & $0.6636(8)$ & $0.8366(4)$ & $0.3007(7)$ & $0.171(5)$ \\
\hline $\mathrm{H} 56 \mathrm{a}$ & $0.7221(8)$ & $0.8363(4)$ & $0.3345(7)$ & 0.228 \\
\hline $\mathrm{H} 56 \mathrm{~b}$ & $0.6803(8)$ & $0.8481(4)$ & $0.2417(7)$ & 0.228 \\
\hline O9 & $0.8841(7)$ & $0.2004(5)$ & $0.3470(7)$ & $0.191(4)$ \\
\hline C57 & $0.8210(6)$ & $0.1951(6)$ & $0.4104(5)$ & $0.139(4)$ \\
\hline H57 & $0.8144(6)$ & $0.2543(6)$ & $0.4343(5)$ & 0.185 \\
\hline C58 & $0.7232(7)$ & $0.1673(9)$ & $0.3792(5)$ & $0.176(5)$ \\
\hline $\mathrm{H} 58 \mathrm{a}$ & $0.7282(7)$ & $0.1111(9)$ & $0.3504(5)$ & 0.235 \\
\hline H58b & $0.6997(7)$ & $0.2099(9)$ & $0.3380(5)$ & 0.235 \\
\hline C59 & $0.6515(6)$ & $0.1593(9)$ & $0.4523(7)$ & $0.192(6)$ \\
\hline $\mathrm{H} 59 \mathrm{a}$ & $0.6395(6)$ & $0.2170(9)$ & $0.4763(7)$ & 0.255 \\
\hline $\mathrm{H} 59 \mathrm{~b}$ & $0.5914(6)$ & $0.1366(9)$ & $0.4300(7)$ & 0.255 \\
\hline C60 & $0.6891(9)$ & $0.0982(8)$ & $0.5226(6)$ & $0.214(7)$ \\
\hline $\mathrm{H} 60 \mathrm{a}$ & $0.6963(9)$ & $0.0391(8)$ & $0.5002(6)$ & 0.285 \\
\hline $\mathrm{H} 60 \mathrm{~b}$ & $0.6441(9)$ & $0.0961(8)$ & $0.5693(6)$ & 0.285 \\
\hline C61 & $0.7860(10)$ & $0.1322(12)$ & $0.5554(6)$ & $0.31(2)$ \\
\hline $\mathrm{H} 61 \mathrm{a}$ & $0.8103(10)$ & $0.0934(12)$ & $0.5998(6)$ & 0.416 \\
\hline $\mathrm{H} 61 \mathrm{~b}$ & $0.7780(10)$ & $0.1903(12)$ & $0.5800(6)$ & 0.416 \\
\hline C62 & $0.8581(6)$ & $0.1368(8)$ & $0.4815(7)$ & $0.208(8)$ \\
\hline $\mathrm{H} 62 \mathrm{a}$ & $0.9184(6)$ & $0.1596(8)$ & $0.5029(7)$ & 0.277 \\
\hline $\mathrm{H} 62 \mathrm{~b}$ & $0.8692(6)$ & $0.0781(8)$ & $0.4595(7)$ & 0.277 \\
\hline
\end{tabular}

Table S18. Refined Thermal Parameters (U's) for 9

Atom

$\mathrm{U}_{11}$

$\mathrm{U}_{22}$

$\mathrm{U}_{33}$

$\mathrm{U}_{23}$

$\mathrm{U}_{13}$

$\mathrm{U}_{12}$ 


\begin{tabular}{|c|c|c|c|c|c|c|}
\hline Ti1 & $0.0610(5)$ & $0.0584(5)$ & $0.0589(4)$ & $0.0022(4)$ & $0.0006(4)$ & $-0.0027(4)$ \\
\hline $\mathrm{Ti} 2$ & $0.0508(4)$ & $0.0550(4)$ & $0.0711(5)$ & $-0.0037(4)$ & $0.0043(3)$ & $0.0008(4)$ \\
\hline 01 & $0.058(2)$ & $0.054(2)$ & $0.065(2)$ & $-0.0065(14)$ & $0.0066(14)$ & $-0.0061(14)$ \\
\hline $\mathrm{O} 2$ & $0.068(2)$ & $0.065(2)$ & $0.067(2)$ & $0.000(2)$ & $-0.003(2)$ & $0.006(2)$ \\
\hline $\mathrm{O} 3$ & $0.064(2)$ & $0.073(2)$ & $0.103(3)$ & $0.006(2)$ & $-0.015(2)$ & $0.007(2)$ \\
\hline O4 & $0.111(3)$ & $0.099(3)$ & $0.055(2)$ & $-0.001(2)$ & $0.003(2)$ & $-0.025(2)$ \\
\hline O5 & $0.053(2)$ & $0.056(2)$ & $0.074(2)$ & $0.001(2)$ & $0.002(2)$ & $-0.0075(13)$ \\
\hline O6 & $0.063(2)$ & $0.090(3)$ & $0.081(2)$ & $0.020(2)$ & $0.006(2)$ & $0.000(2)$ \\
\hline $\mathrm{O} 7$ & $0.055(2)$ & $0.079(3)$ & $0.091(3)$ & $0.010(2)$ & $0.002(2)$ & $-0.002(2)$ \\
\hline O8 & $0.080(3)$ & $0.077(3)$ & $0.132(4)$ & $-0.038(3)$ & $0.007(2)$ & $0.016(2)$ \\
\hline C1 & $0.059(3)$ & $0.056(3)$ & $0.053(2)$ & $0.003(2)$ & $0.006(2)$ & $-0.012(2)$ \\
\hline C2 & $0.065(3)$ & $0.074(3)$ & $0.057(3)$ & $-0.004(2)$ & $0.010(2)$ & $-0.008(2)$ \\
\hline C3 & $0.068(3)$ & $0.094(4)$ & $0.062(3)$ & $0.008(3)$ & $0.011(2)$ & $-0.017(3)$ \\
\hline C4 & $0.075(3)$ & $0.074(3)$ & $0.060(3)$ & $0.006(2)$ & $-0.004(2)$ & $-0.022(3)$ \\
\hline C5 & $0.117(5)$ & $0.108(5)$ & $0.083(4)$ & $0.014(4)$ & $-0.005(4)$ & $-0.055(4)$ \\
\hline C6 & $0.123(7)$ & $0.079(5)$ & $0.235(12)$ & $-0.013(6)$ & $0.021(7)$ & $-0.036(5)$ \\
\hline C7 & $0.195(9)$ & $0.082(4)$ & $0.116(6)$ & $-0.007(4)$ & $0.033(6)$ & $-0.050(6)$ \\
\hline C8 & $0.105(4)$ & $0.061(3)$ & $0.079(4)$ & $-0.005(3)$ & $-0.013(3)$ & $-0.017(3)$ \\
\hline C9 & $0.074(3)$ & $0.059(3)$ & $0.057(3)$ & $0.002(2)$ & $-0.006(2)$ & $-0.015(2)$ \\
\hline C10 & $0.059(3)$ & $0.051(2)$ & $0.052(2)$ & $0.002(2)$ & $-0.001(2)$ & $-0.002(2)$ \\
\hline C11 & $0.067(3)$ & $0.051(2)$ & $0.051(2)$ & $0.003(2)$ & $0.008(2)$ & $0.000(2)$ \\
\hline C12 & $0.077(3)$ & $0.050(2)$ & $0.056(2)$ & $-0.001(2)$ & $0.011(2)$ & $0.002(2)$ \\
\hline C13 & $0.083(3)$ & $0.068(3)$ & $0.057(3)$ & $-0.005(2)$ & $0.000(2)$ & $0.006(3)$ \\
\hline C14 & $0.101(5)$ & $0.144(6)$ & $0.059(3)$ & $0.015(4)$ & $-0.001(3)$ & $0.010(4)$ \\
\hline C15 & $0.146(8)$ & $0.225(12)$ & $0.060(4)$ & $0.027(5)$ & $0.007(4)$ & $0.045(8)$ \\
\hline C16 & $0.127(6)$ & $0.099(4)$ & $0.061(3)$ & $0.006(3)$ & $0.027(4)$ & $0.019(4)$ \\
\hline C17 & $0.095(4)$ & $0.067(3)$ & $0.063(3)$ & $-0.001(2)$ & $0.019(3)$ & $0.019(3)$ \\
\hline C18 & $0.089(4)$ & $0.100(4)$ & $0.073(4)$ & $-0.005(3)$ & $0.035(3)$ & $0.007(3)$ \\
\hline C19 & $0.065(3)$ & $0.083(4)$ & $0.082(4)$ & $-0.006(3)$ & $0.020(3)$ & $0.009(3)$ \\
\hline C20 & $0.077(3)$ & $0.052(2)$ & $0.068(3)$ & $-0.008(2)$ & $0.004(2)$ & $0.006(2)$ \\
\hline C22 & $0.081(5)$ & $0.198(10)$ & $0.132(7)$ & $0.064(7)$ & $-0.002(4)$ & $0.040(6)$ \\
\hline C25 & $0.091(6)$ & $0.184(11)$ & $0.234(13)$ & $0.029(11)$ & $0.021(8)$ & $0.055(7)$ \\
\hline C21 & $0.063(6)$ & $0.091(8)$ & $0.123(9)$ & $0.062(7)$ & $0.002(6)$ & $0.011(6)$ \\
\hline C23 & $0.090(11)$ & $0.26(3)$ & $0.17(2)$ & $0.14(2)$ & $0.007(11)$ & $0.007(14)$ \\
\hline C24 & $0.102(13)$ & $0.091(12)$ & $0.31(3)$ & $-0.022(14)$ & $-0.06(2)$ & $0.044(10)$ \\
\hline C26 & $0.076(9)$ & $0.18(2)$ & $0.146(13)$ & $-0.057(12)$ & $-0.005(8)$ & $0.012(10)$ \\
\hline C21' & $0.066(11)$ & $0.10(2)$ & $0.18(2)$ & $0.08(2)$ & $0.034(12)$ & $0.022(10)$ \\
\hline C23' & $0.070(13)$ & $0.32(5)$ & $0.42(6)$ & $0.32(5)$ & $0.09(2)$ & $0.09(2)$ \\
\hline C24' & $0.080(14)$ & $0.09(2)$ & $0.23(3)$ & $-0.04(2)$ & $0.00(2)$ & $0.030(11)$ \\
\hline C26' & $0.039(7)$ & $0.14(2)$ & $0.12(2)$ & $-0.059(13)$ & $-0.009(8)$ & $0.009(9)$ \\
\hline C27 & $0.151(6)$ & $0.103(5)$ & $0.068(3)$ & $-0.007(4)$ & $0.026(4)$ & $0.019(5)$ \\
\hline C30 & $0.195(11)$ & $0.198(12)$ & $0.089(6)$ & $-0.041(7)$ & $0.049(7)$ & $-0.003(9)$ \\
\hline C28 & $0.107(8)$ & $0.129(10)$ & $0.072(6)$ & $-0.024(6)$ & $-0.009(6)$ & $-0.023(7)$ \\
\hline C29 & $0.144(12)$ & $0.141(12)$ & $0.094(9)$ & $-0.037(8)$ & $-0.039(9)$ & $0.018(10)$ \\
\hline C31 & $0.17(2)$ & $0.19(2)$ & $0.059(7)$ & $0.024(10)$ & $0.017(8)$ & $-0.031(14)$ \\
\hline C32 & $0.097(8)$ & $0.165(14)$ & $0.106(9)$ & $-0.034(10)$ & $0.011(7)$ & $-0.005(9)$ \\
\hline C28' & $0.099(10)$ & $0.087(10)$ & $0.070(8)$ & $0.029(7)$ & $0.022(8)$ & $0.042(8)$ \\
\hline C29' & $0.32(4)$ & $0.15(2)$ & $0.11(2)$ & $0.06(2)$ & $0.13(2)$ & $0.14(2)$ \\
\hline C31' & $0.19(2)$ & $0.098(13)$ & $0.053(8)$ & $-0.007(8)$ & $-0.023(11)$ & $0.05(2)$ \\
\hline C32' & $0.16(2)$ & $0.107(12)$ & $0.052(8)$ & $0.013(8)$ & $-0.003(9)$ & $0.059(12)$ \\
\hline C33 & $0.053(3)$ & $0.062(3)$ & $0.077(3)$ & $0.002(2)$ & $0.006(2)$ & $-0.013(2)$ \\
\hline C34 & $0.067(3)$ & $0.078(3)$ & $0.071(3)$ & $-0.009(3)$ & $0.008(2)$ & $-0.019(3)$ \\
\hline C35 & $0.082(4)$ & $0.095(4)$ & $0.089(4)$ & $-0.010(3)$ & $0.011(3)$ & $-0.037(3)$ \\
\hline C36 & $0.082(4)$ & $0.099(5)$ & $0.113(5)$ & $0.000(4)$ & $0.008(4)$ & $-0.034(4)$ \\
\hline C37 & $0.067(3)$ & $0.084(4)$ & $0.083(4)$ & $0.006(3)$ & $0.014(3)$ & $-0.004(3)$ \\
\hline C38 & $0.060(3)$ & $0.065(3)$ & $0.064(3)$ & $0.002(2)$ & $0.005(2)$ & $-0.001(2)$ \\
\hline C39 & $0.089(4)$ & $0.080(4)$ & $0.090(4)$ & $0.025(3)$ & $0.019(3)$ & $0.010(3)$ \\
\hline C40 & $0.141(7)$ & $0.113(6)$ & $0.113(6)$ & $0.010(5)$ & $0.055(5)$ & $0.019(5)$ \\
\hline
\end{tabular}




\begin{tabular}{|c|c|c|c|c|c|c|}
\hline C44 & $0.140(7)$ & $0.086(5)$ & $0.130(6)$ & $0.031(4)$ & $0.045(5)$ & $0.027(5)$ \\
\hline C41 & $0.102(9)$ & $0.126(11)$ & $0.135(11)$ & $0.022(9)$ & $0.036(8)$ & $-0.014(8)$ \\
\hline C42 & $0.159(14)$ & $0.091(9)$ & $0.134(12)$ & $0.044(9)$ & $0.024(10)$ & $-0.015(9)$ \\
\hline $\mathrm{C} 43$ & $0.155(13)$ & $0.083(8)$ & $0.123(10)$ & $0.020(7)$ & $0.040(9)$ & $-0.004(8)$ \\
\hline C41' & $0.122(14)$ & $0.16(2)$ & $0.066(9)$ & $0.020(10)$ & $0.019(9)$ & $0.076(13)$ \\
\hline C42' & $0.16(2)$ & $0.16(2)$ & $0.080(11)$ & $0.056(13)$ & $0.040(13)$ & $0.06(2)$ \\
\hline C43' & $0.14(2)$ & $0.11(2)$ & $0.12(2)$ & $0.051(13)$ & $0.010(13)$ & $0.047(13)$ \\
\hline C45 & $0.052(3)$ & $0.074(3)$ & $0.085(4)$ & $0.002(3)$ & $-0.001(2)$ & $-0.003(2)$ \\
\hline C46 & $0.073(4)$ & $0.145(7)$ & $0.102(5)$ & $-0.031(5)$ & $0.023(3)$ & $0.005(4)$ \\
\hline C47 & $0.070(4)$ & $0.193(10)$ & $0.149(8)$ & $-0.019(7)$ & $0.037(5)$ & $-0.013(5)$ \\
\hline C48 & $0.053(3)$ & $0.129(6)$ & $0.170(8)$ & $-0.009(6)$ & $-0.001(4)$ & $0.000(4)$ \\
\hline C49 & $0.075(4)$ & $0.118(5)$ & $0.125(6)$ & $0.012(5)$ & $-0.029(4)$ & $-0.013(4)$ \\
\hline C50 & $0.060(3)$ & $0.097(4)$ & $0.094(4)$ & $0.009(3)$ & $-0.004(3)$ & $0.006(3)$ \\
\hline C51 & $0.090(4)$ & $0.067(3)$ & $0.113(5)$ & $-0.019(3)$ & $0.014(4)$ & $0.003(3)$ \\
\hline C52 & $0.161(9)$ & $0.094(6)$ & $0.164(9)$ & $0.011(6)$ & $0.047(7)$ & $-0.009(6)$ \\
\hline C53 & $0.140(9)$ & $0.207(14)$ & $0.223(14)$ & $-0.087(11)$ & $0.060(9)$ & $-0.010(9)$ \\
\hline C54 & $0.161(10)$ & $0.174(11)$ & $0.181(11)$ & $-0.088(9)$ & $0.003(8)$ & $-0.022(8)$ \\
\hline C55 & $0.27(2)$ & $0.066(5)$ & $0.233(14)$ & $-0.007(7)$ & $-0.038(12)$ & $0.043(7)$ \\
\hline C56 & $0.199(12)$ & $0.083(5)$ & $0.234(13)$ & $-0.010(6)$ & $0.092(10)$ & $-0.036(6)$ \\
\hline O9 & $0.189(8)$ & $0.114(5)$ & $0.268(11)$ & $0.067(6)$ & $-0.074(8)$ & $-0.013(5)$ \\
\hline C57 & $0.194(11)$ & $0.102(6)$ & $0.119(7)$ & $0.018(5)$ & $-0.059(7)$ & $-0.011(7)$ \\
\hline C58 & $0.190(11)$ & $0.220(13)$ & $0.118(8)$ & $0.034(8)$ & $-0.078(8)$ & $-0.036(10)$ \\
\hline C59 & $0.26(2)$ & $0.194(13)$ & $0.120(8)$ & $0.038(9)$ & $-0.040(9)$ & $-0.012(12)$ \\
\hline C60 & $0.39(2)$ & $0.150(10)$ & $0.101(7)$ & $0.029(7)$ & $-0.046(10)$ & $-0.084(13)$ \\
\hline C61 & $0.56(4)$ & $0.25(2)$ & $0.129(11)$ & $0.019(12)$ & $-0.13(2)$ & $-0.01(2)$ \\
\hline C62 & $0.24(2)$ & $0.170(11)$ & $0.214(14)$ & $0.049(10)$ & $-0.156(13)$ & $-0.028(10)$ \\
\hline
\end{tabular}

Table S19. Bond Distances in 9, $\AA$

\begin{tabular}{|llllll|}
\hline Ti1-O4 & $1.754(4)$ & Ti1-O3 & $1.775(4)$ & Ti1-O2 & $1.885(4)$ \\
Ti1-O5 & $1.923(3)$ & Ti1-O1 & $2.139(3)$ & Ti1-Ti2 & $3.3224(12)$ \\
Ti2-O7 & $1.769(4)$ & Ti2-O8 & $1.785(4)$ & Ti2-O6 & $1.800(4)$ \\
Ti2-O1 & $1.976(3)$ & Ti2-O5 & $2.122(3)$ & O1-C1 & $1.382(5)$ \\
O2-C20 & $1.390(6)$ & O3-C21 & $1.414(9)$ & O3-C21' & $1.433(14)$ \\
O4-C27 & $1.317(6)$ & O5-C33 & $1.455(5)$ & O6-C39 & $1.419(7)$ \\
O7-C45 & $1.397(6)$ & O8-C51 & $1.381(6)$ & C1-C10 & $1.381(6)$ \\
C1-C2 & $1.407(6)$ & C2-C3 & $1.392(8)$ & C3-C4 & $1.366(8)$ \\
C4-C9 & $1.402(7)$ & C4-C5 & $1.513(8)$ & C5-C6 & $1.475(12)$ \\
C6-C7 & $1.449(12)$ & C7-C8 & $1.503(9)$ & C8-C9 & $1.529(7)$ \\
C9-C10 & $1.392(6)$ & C10-C11 & $1.490(6)$ & C11-C20 & $1.391(7)$ \\
C11-C12 & $1.412(7)$ & C12-C17 & $1.407(7)$ & C12-C13 & $1.492(7)$ \\
C13-C14 & $1.489(8)$ & C14-C15 & $1.375(11)$ & C15-C16 & $1.492(12)$ \\
C16-C17 & $1.514(8)$ & C17-C18 & $1.416(9)$ & C18-C19 & $1.324(8)$ \\
C19-C20 & $1.390(7)$ & C22-C21 & $1.528(8)$ & C22-C23' & $1.540(9)$ \\
C22-C23 & $1.547(8)$ & C22-C21' & $1.549(9)$ & C25-C24' & $1.536(9)$ \\
C25-C24 & $1.541(9)$ & C25-C26 & $1.543(8)$ & C25-C26' & $1.543(9)$ \\
C21-C26 & $1.544(9)$ & C23-C24 & $1.532(9)$ & C21'-C26' & $1.536(9)$ \\
C23'-C24' & $1.540(9)$ & C27-C32' & $1.432(7)$ & C27-C32 & $1.483(7)$ \\
C27-C28' & $1.508(7)$ & C27-C28 & $1.527(7)$ & C30-C31' & $1.515(9)$ \\
C30-C31 & $1.552(9)$ & C30-C29' & $1.567(9)$ & C30-C29 & $1.581(8)$ \\
C28-C29 & $1.537(8)$ & C31-C32 & $1.525(8)$ & C28'-C29' & $1.489(8)$ \\
C31'-C32' & $1.512(9)$ & C33-C34 & $1.506(8)$ & C33-C38 & $1.526(7)$ \\
C34-C35 & $1.519(8)$ & C35-C36 & $1.540(9)$ & C36-C37 & $1.519(9)$ \\
C37-C38 & $1.507(8)$ & C39-C40 & $1.500(6)$ & C39-C44 & $1.517(6)$ \\
C40-C41' & $1.486(8)$ & C40-C41 & $1.499(8)$ & C44-C43' & $1.498(8)$ \\
C44-C43 & $1.497(8)$ & C41-C42 & $1.530(8)$ & C42-C43 & $1.554(8)$ \\
\hline
\end{tabular}




\begin{tabular}{|llllll|}
\hline C41'-C42' & $1.534(9)$ & C42'-C43' $^{\prime}$ & $1.535(9)$ & C45-C50 & $1.502(8)$ \\
C45-C46 & $1.510(8)$ & C46-C47 & $1.522(10)$ & C47-C48 & $1.481(12)$ \\
C48-C49 & $1.474(11)$ & C49-C50 & $1.509(8)$ & C51-C56 & $1.521(7)$ \\
C51-C52 & $1.528(7)$ & C52-C53 & $1.534(8)$ & C53-C54 & $1.531(8)$ \\
C54-C55 & $1.526(8)$ & C55-C56 & $1.517(8)$ & O9-C57 & $1.338(13)$ \\
C57-C58 & $1.509(8)$ & C57-C62 & $1.511(8)$ & C58-C59 & $1.534(8)$ \\
C59-C60 & $1.529(8)$ & C60-C61 & $1.535(9)$ & C61-C62 & $1.543(9)$ \\
\hline
\end{tabular}

Table S20. Bond Angles in 9,

\begin{tabular}{|c|c|c|c|c|c|}
\hline O4-Ti1-O3 & 102.1(2) & O4-Ti1-O2 & 108.9(2) & O3-Ti1-O2 & $96.0(2)$ \\
\hline O4-Ti1-O5 & $116.0(2)$ & O3-Ti1-O5 & $97.7(2)$ & O2-Ti1-O5 & $128.64(14)$ \\
\hline O4-Ti1-O1 & $91.6(2)$ & O3-Ti1-O1 & $165.4(2)$ & O2-Ti1-O1 & $84.26(13)$ \\
\hline O5-Ti1-O1 & 71.32(12) & O4-Ti1-Ti2 & $106.4(2)$ & O3-Ti1-Ti2 & $133.72(14)$ \\
\hline O2-Ti1-Ti2 & 107.99(11) & O5-Ti1-Ti2 & $36.80(9)$ & O1-Ti1-Ti2 & $34.52(9)$ \\
\hline O7-Ti2-O8 & $101.1(2)$ & O7-Ti2-O6 & $99.6(2)$ & O8-Ti2-06 & $112.3(2)$ \\
\hline O7-Ti2-O1 & $92.7(2)$ & O8-Ti2-O1 & $118.5(2)$ & O6-Ti2-O1 & 123.9(2) \\
\hline O7-Ti2-O5 & 163.3(2) & O8-Ti2-O5 & $88.6(2)$ & O6-Ti2-O5 & $88.9(2)$ \\
\hline O1-Ti2-O5 & $70.71(13)$ & O7-Ti2-Ti1 & $130.51(13)$ & O8-Ti2-Ti1 & $105.1(2)$ \\
\hline O6-Ti2-Ti1 & $107.89(12)$ & O1-Ti2-Ti1 & $37.84(10)$ & O5-Ti2-Ti1 & $32.87(8)$ \\
\hline C1-O1-Ti2 & $130.4(3)$ & C1-O1-Ti1 & $121.7(3)$ & Ti2-O1-Ti1 & $107.64(14)$ \\
\hline C20-O2-Ti1 & $117.2(3)$ & C21-O3-Ti1 & $159.4(6)$ & C21'-O3-Ti1 & $129.6(8)$ \\
\hline C27-O4-Ti1 & $157.8(5)$ & C33-O5-Ti1 & $134.3(3)$ & C33-O5-Ti2 & $115.4(3)$ \\
\hline Ti1-O5-Ti2 & 110.33(14) & С39-O6-Ti2 & 137.7(3) & C45-O7-Ti2 & 169.1(4) \\
\hline C51-O8-Ti2 & $142.8(4)$ & C10-C1-O1 & $119.4(4)$ & C10-C1-C2 & $121.9(4)$ \\
\hline O1-C1-C2 & $118.6(4)$ & C3-C2-C1 & 116.5(5) & C4-C3-C2 & 123.1(5) \\
\hline C3-C4-C9 & $119.0(5)$ & C3-C4-C5 & $119.5(5)$ & C9-C4-C5 & $121.4(5)$ \\
\hline C6-C5-C4 & $115.2(6)$ & C7-C6-C5 & 119.3(7) & C6-C7-C8 & 114.1(8) \\
\hline C7-C8-C9 & $114.0(6)$ & C10-C9-C4 & $120.0(5)$ & C10-C9-C8 & $119.2(5)$ \\
\hline C4-C9-C8 & $120.5(4)$ & C1-C10-C9 & $119.3(4)$ & C1-C10-C11 & $118.0(4)$ \\
\hline C9-C10-C11 & $122.6(4)$ & C20-C11-C12 & $118.9(4)$ & C20-C11-C10 & $119.2(4)$ \\
\hline C12-C11-C10 & $121.7(4)$ & C17-C12-C11 & $119.0(5)$ & C17-C12-C13 & $120.5(5)$ \\
\hline C11-C12-C13 & $120.5(4)$ & C14-C13-C12 & $115.1(5)$ & C15-C14-C13 & 116.1(6) \\
\hline C14-C15-C16 & 119.0(8) & C15-C16-C17 & $112.8(6)$ & C12-C17-C18 & $119.0(5)$ \\
\hline C12-C17-C16 & $121.4(6)$ & C18-C17-C16 & $119.5(5)$ & C19-C18-C17 & $121.5(5)$ \\
\hline C18-C19-C20 & $120.5(6)$ & O2-C20-C11 & $119.0(4)$ & O2-C20-C19 & $120.0(5)$ \\
\hline C11-C20-C19 & $120.9(5)$ & C21-C22-C23 & $109.4(6)$ & C23'-C22-C21' & $109.0(7)$ \\
\hline C24-C25-C26 & $109.1(6)$ & C24'-C25-C26' & $109.7(7)$ & O3-C21-C22 & $109.9(7)$ \\
\hline O3-C21-C26 & $108.3(8)$ & C22-C21-C26 & $109.0(6)$ & C24-C23-C22 & $109.7(7)$ \\
\hline C23-C24-C25 & $109.9(7)$ & C25-C26-C21 & $108.8(6)$ & O3-C21'-C26' & $106.8(10)$ \\
\hline O3-C21'-C22 & 107.7(9) & C26'-C21'-C22 & 109.3(7) & C22-C23'-C24' & $109.5(8)$ \\
\hline C25-C24'-C23' & $109.3(8)$ & C21'-C26'-C25 & $109.1(7)$ & O4-C27-C32' & $133.8(6)$ \\
\hline O4-C27-C32 & $122.5(6)$ & O4-C27-C28' & $105.5(6)$ & C32'-C27-C28' & $120.7(4)$ \\
\hline O4-C27-C28 & $114.7(5)$ & C32-C27-C28 & $115.8(5)$ & С31'-C30-C29' & 108.9(8) \\
\hline C31-C30-C29 & $105.4(6)$ & C27-C28-C29 & $112.3(6)$ & C28-C29-C30 & $106.9(6)$ \\
\hline C32-C31-C30 & $108.2(7)$ & C27-C32-C31 & $113.5(6)$ & C29'-C28'-C27 & $117.7(5)$ \\
\hline C28'-C29'-C30 & $114.4(7)$ & C32'-C31'-C30 & $112.5(8)$ & C27-C32'-C31' & $118.5(6)$ \\
\hline O5-C33-C34 & $110.6(4)$ & O5-C33-C38 & $111.5(4)$ & С34-C33-С38 & $110.9(4)$ \\
\hline C33-C34-C35 & $110.3(5)$ & C34-C35-C36 & 110.1(5) & C37-C36-C35 & $111.5(5)$ \\
\hline C38-C37-C36 & $111.2(5)$ & С37-C38-C33 & $109.8(4)$ & O6-C39-C40 & 108.1(5) \\
\hline
\end{tabular}




\begin{tabular}{|llllll|} 
O6-C39-C44 & $109.0(5)$ & C40-C39-C44 & $115.4(3)$ & C41'-C40-C39 & $116.5(6)$ \\
C41-C40-C39 & $114.8(5)$ & C43'-C44-C39 & $114.3(6)$ & C43-C44-C39 & $113.1(5)$ \\
C40-C41-C42 & $113.5(6)$ & C41-C42-C43 & $108.9(6)$ & C44-C43-C42 & $109.5(6)$ \\
C40-C41'-C42' & $112.6(7)$ & C41'-C42'-C43' & $110.1(8)$ & C44-C43'-C42' & $112.4(7)$ \\
O7-C45-C50 & $111.4(5)$ & O7-C45-C46 & $112.4(5)$ & C50-C45-C46 & $111.1(5)$ \\
C45-C46-C47 & $111.4(6)$ & C48-C47-C46 & $112.4(7)$ & C49-C48-C47 & $112.0(6)$ \\
C48-C49-C50 & $114.0(6)$ & C45-C50-C49 & $111.4(5)$ & O8-C51-C56 & $111.7(5)$ \\
O8-C51-C52 & $110.1(6)$ & C56-C51-C52 & $110.8(5)$ & C51-C52-C53 & $110.8(6)$ \\
C54-C53-C52 & $109.3(6)$ & C55-C54-C53 & $110.6(6)$ & C56-C55-C54 & $112.2(6)$ \\
C55-C56-C51 & $112.2(6)$ & O9-C57-C58 & $112.5(8)$ & O9-C57-C62 & $110.9(8)$ \\
C58-C57-C62 & $112.1(6)$ & C57-C58-C59 & $112.4(6)$ & C60-C59-C58 & $111.1(6)$ \\
C59-C60-C61 & $109.3(7)$ & C60-C61-C62 & $110.4(7)$ & C57-C62-C61 & $110.8(7)$ \\
\hline
\end{tabular}




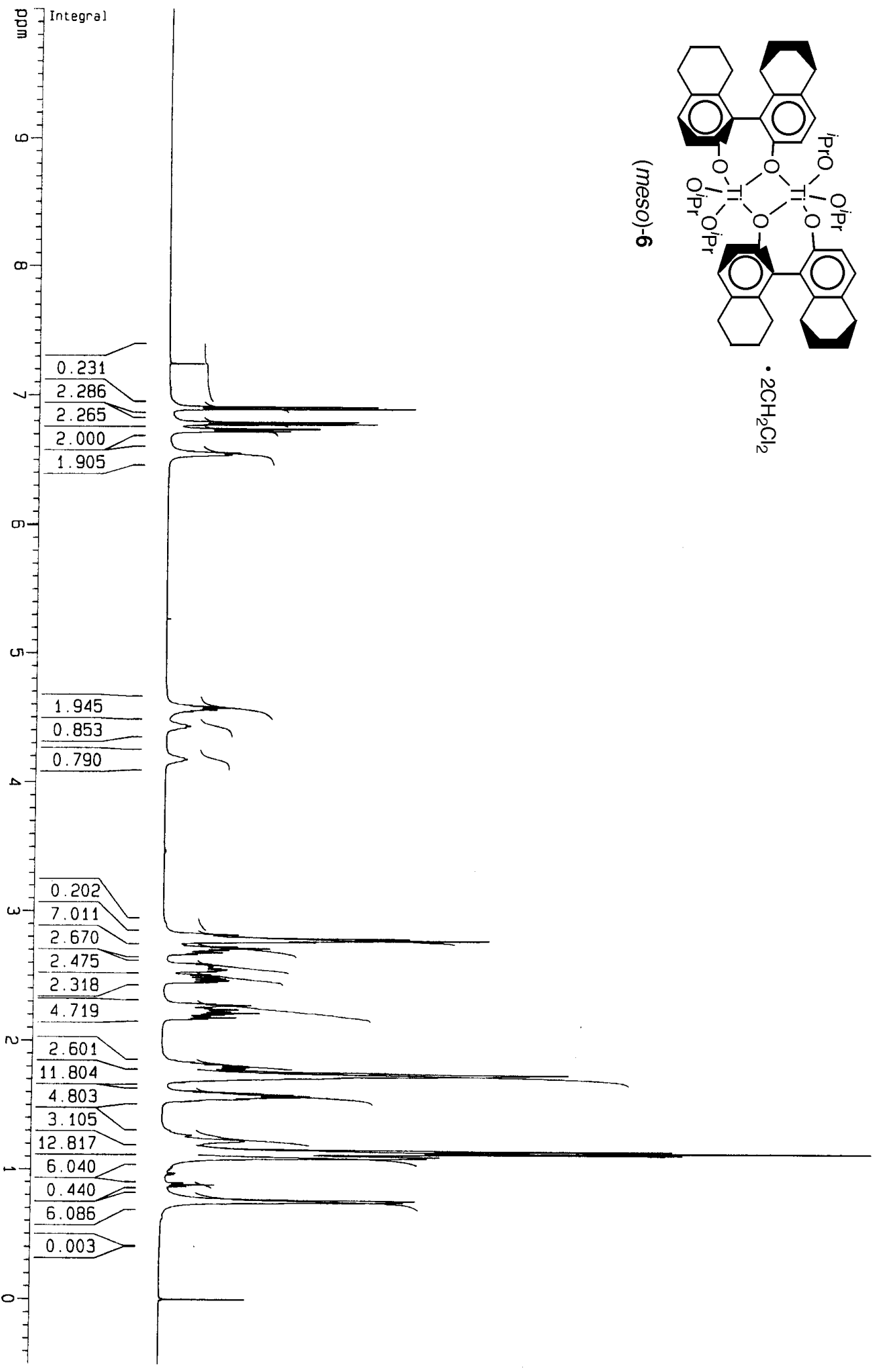

ppm

-... 6.5515 6.5368

[ 4.5944

T 4.5827

4.5705

4.5586

4.5466

$-4.4309$

$-1.1747$

$[2.7852$

to 2.7743

fIr 2.7620

IIF 2.7115

2.2725
-2.2119

UI 2.2119

J -1.7668

1.7596

1.7537

dII 1.7419

1.7357

1.7293

$-1.7240$

1.7153

1. 1.7044

$\Rightarrow 1.5831$

$1-1.5741$

$7\left\lfloor\longleftarrow \begin{array}{r}1.5661 \\ -1.1520\end{array}\right.$

IL 1.1377

- 1.1240

L 1.1031

$-1.0926$

t- 0.7590

-0.7487
$-\quad 0.7590$ 


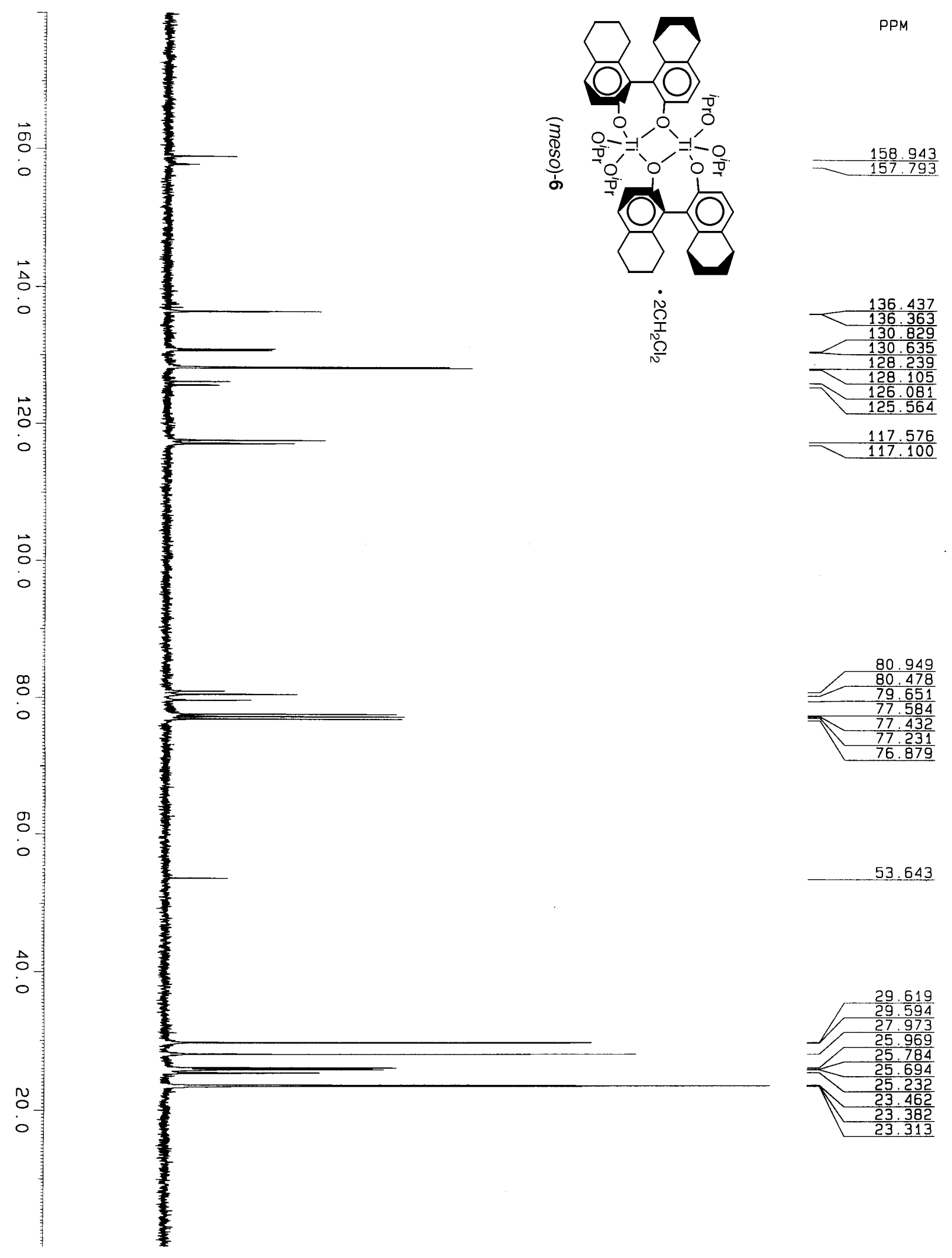




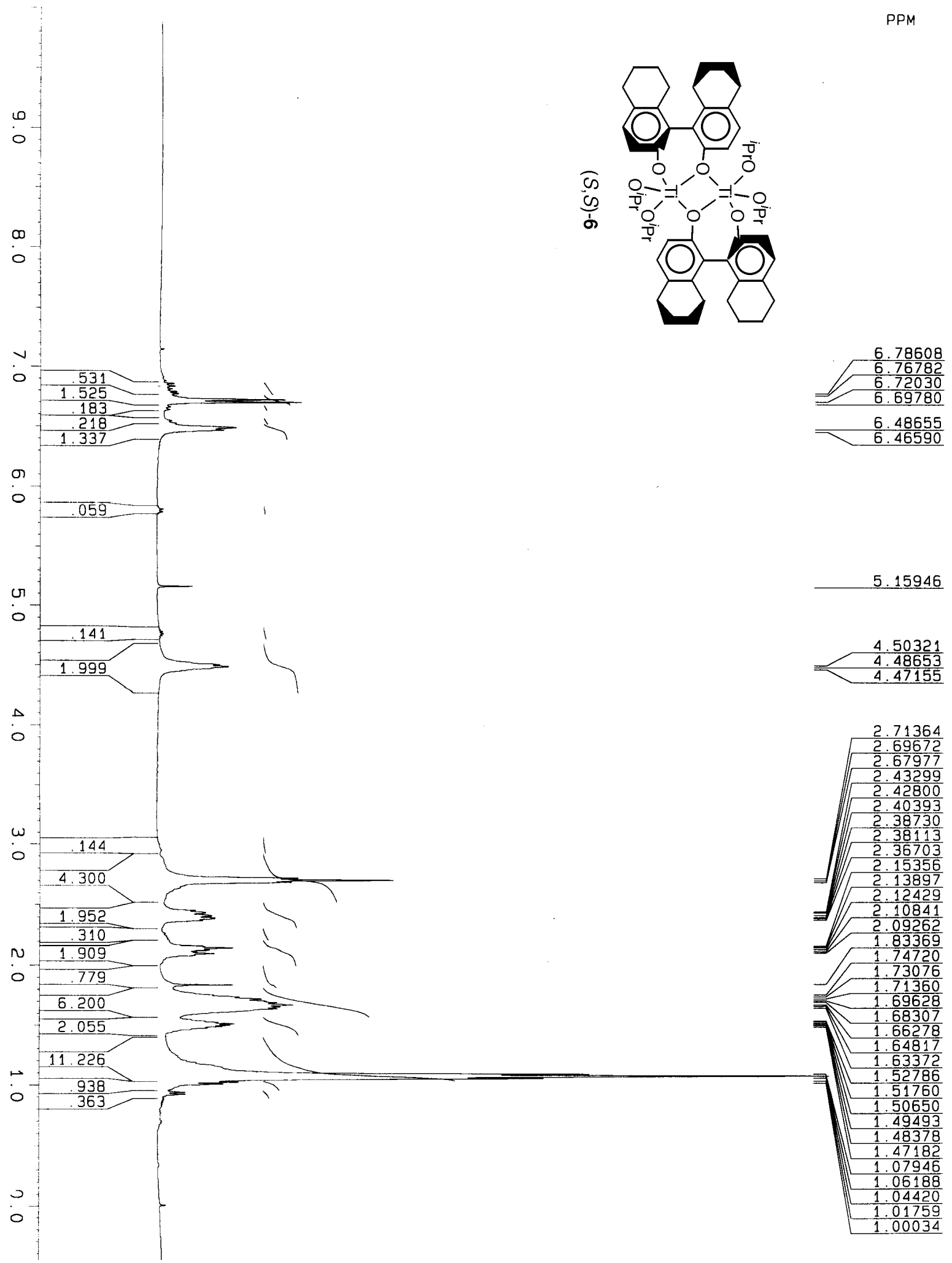




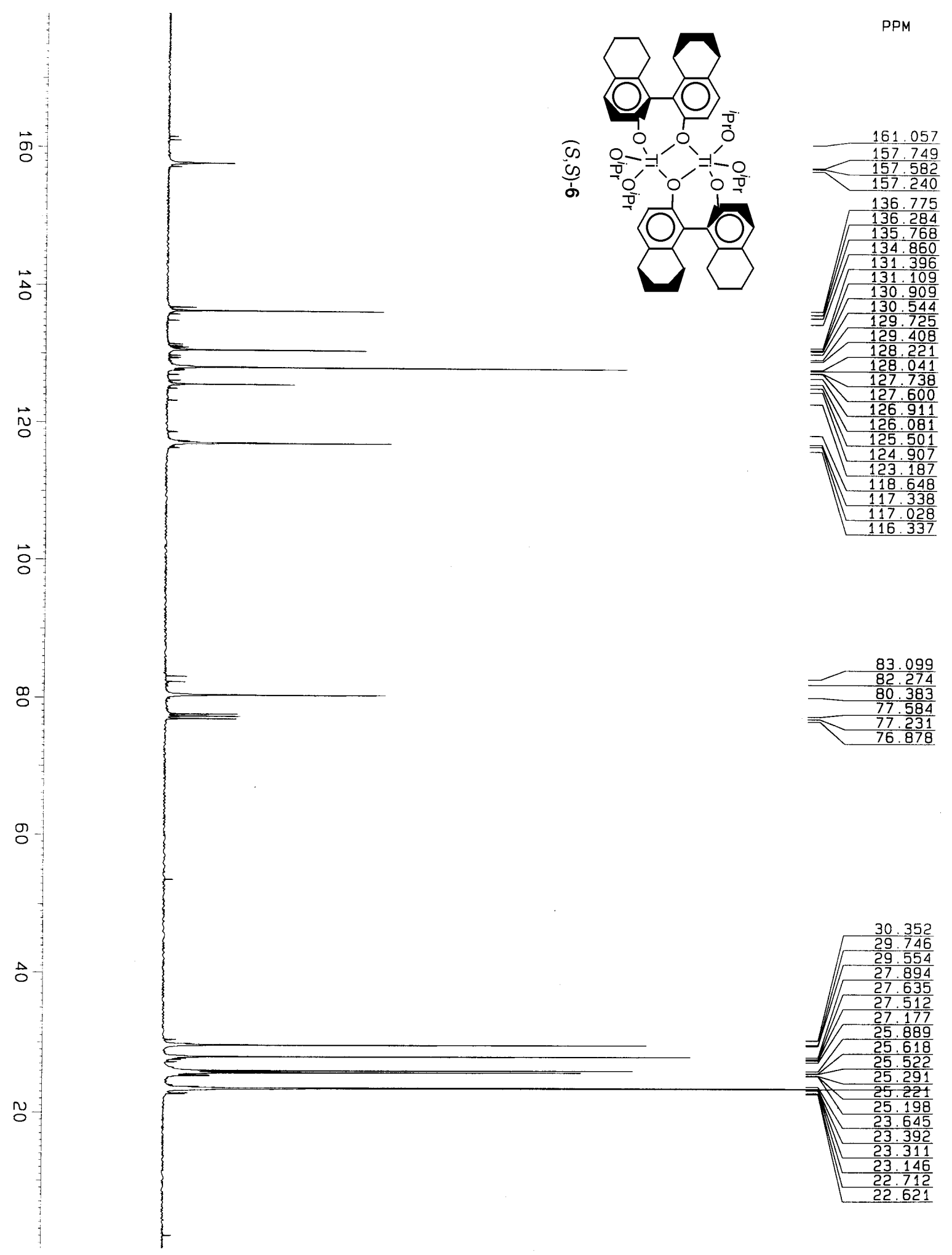




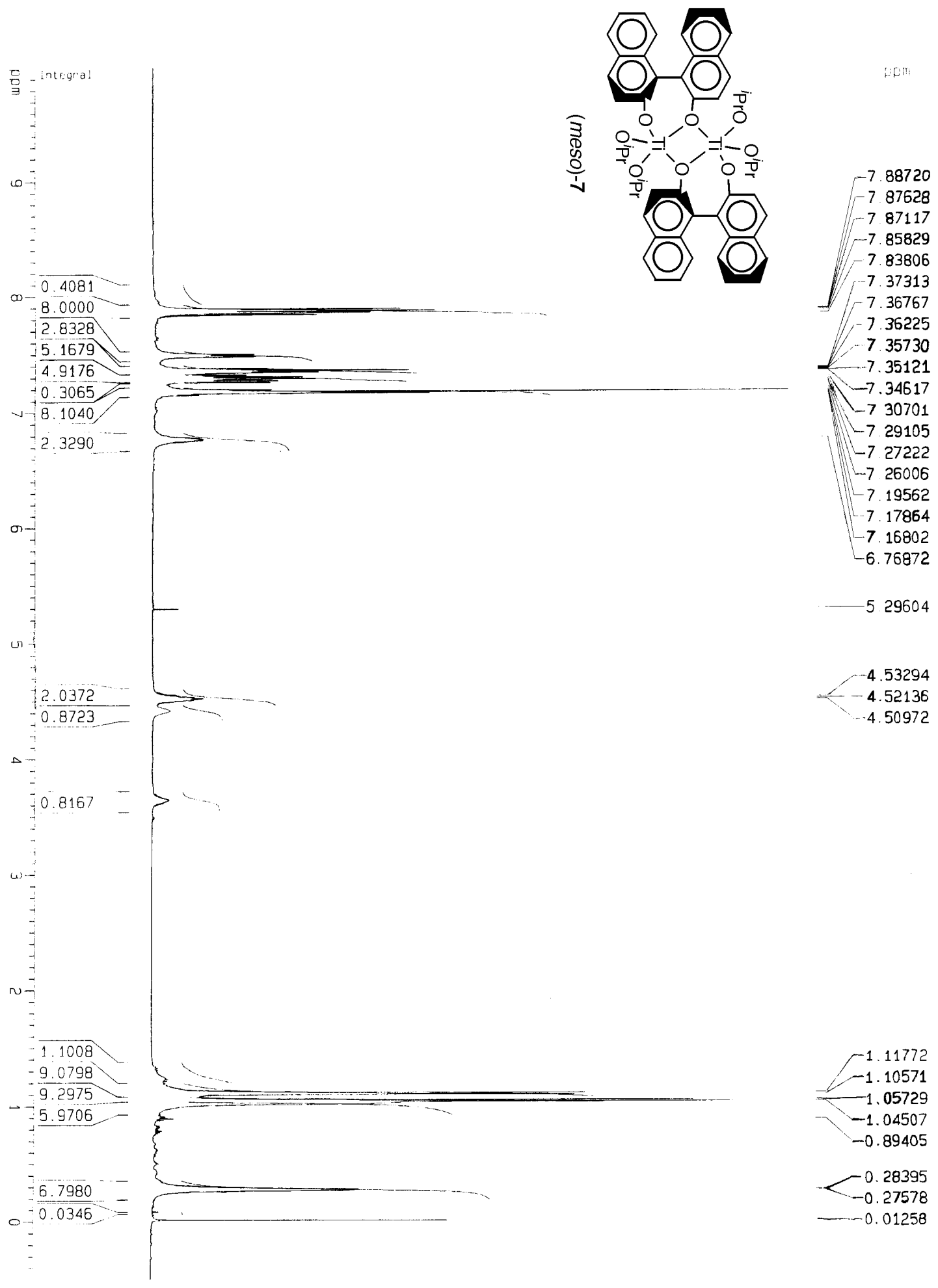




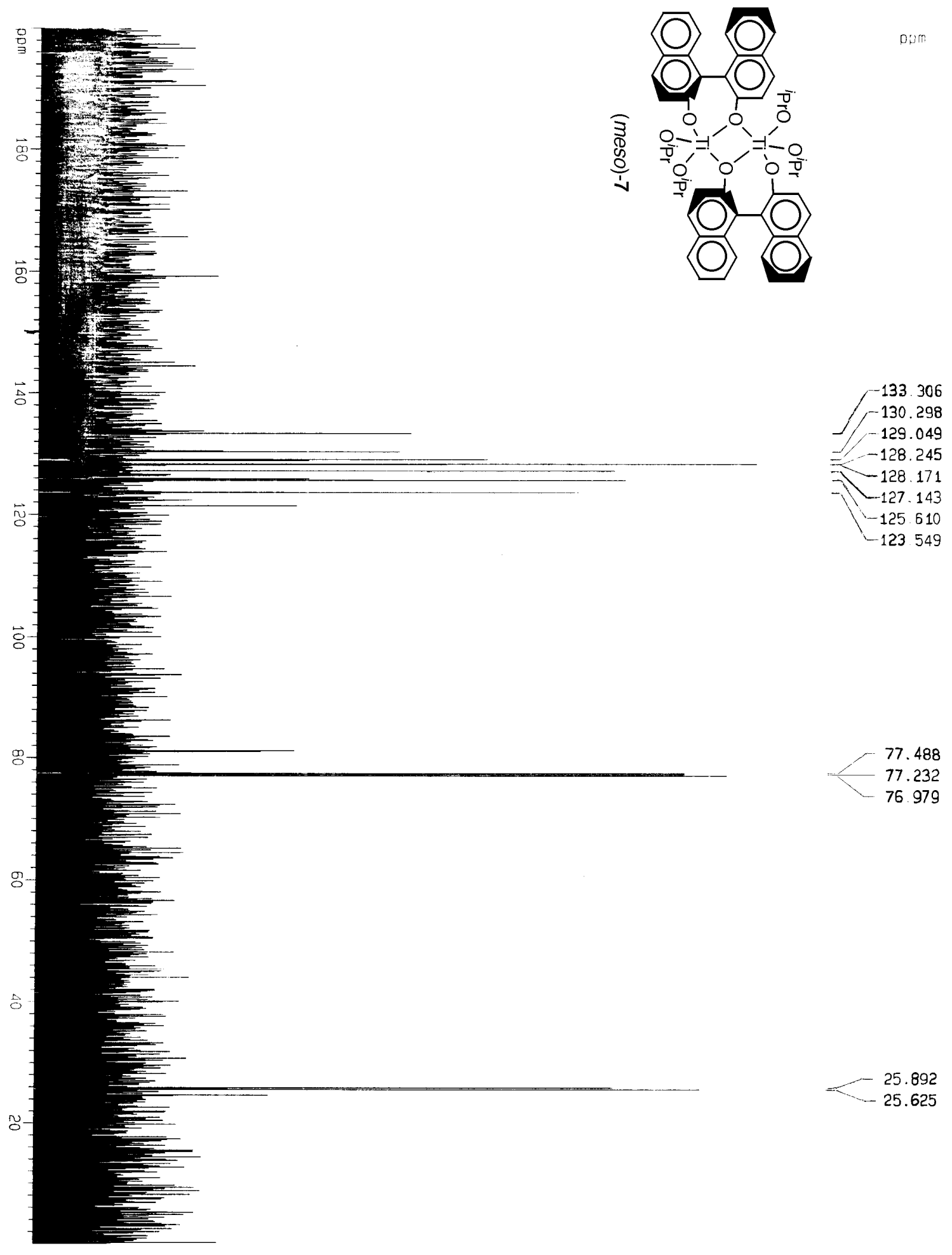




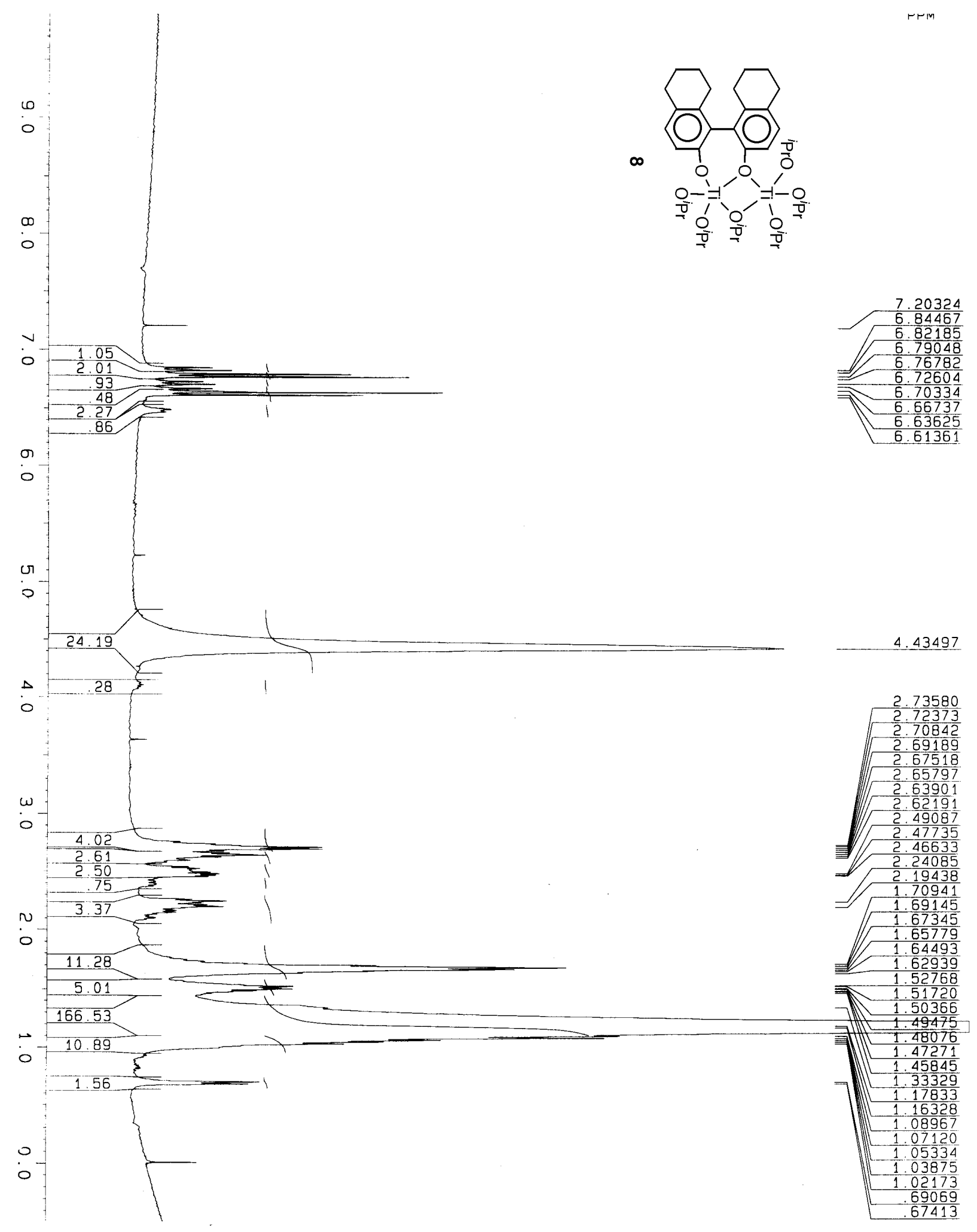




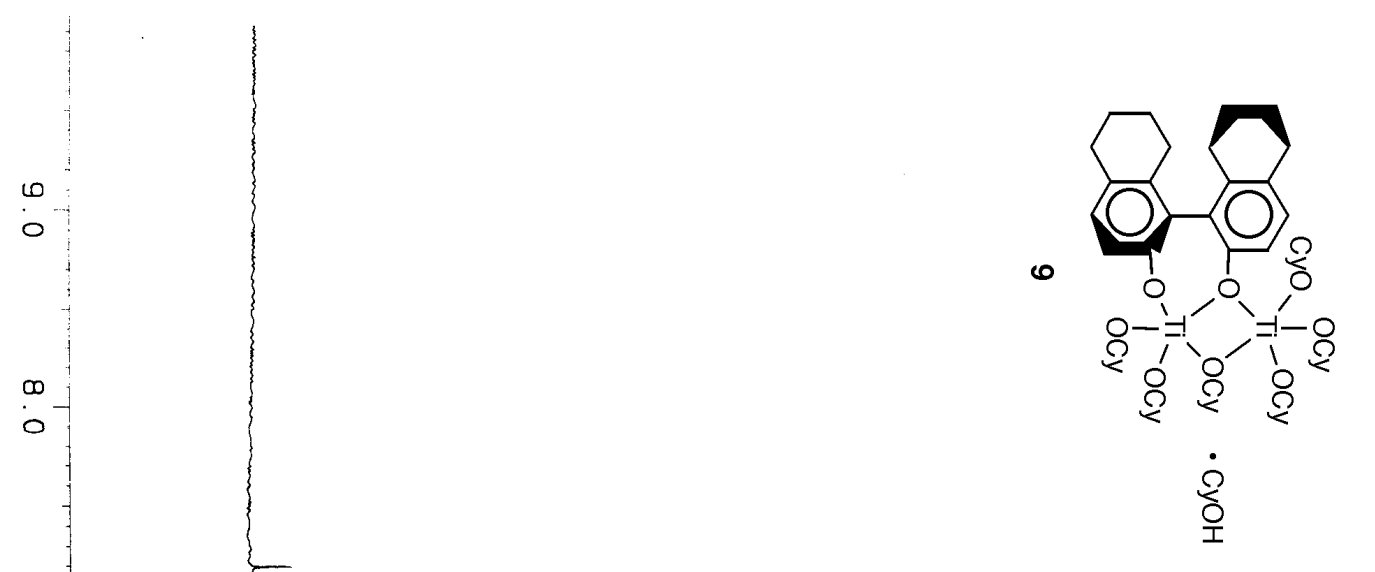

PPM
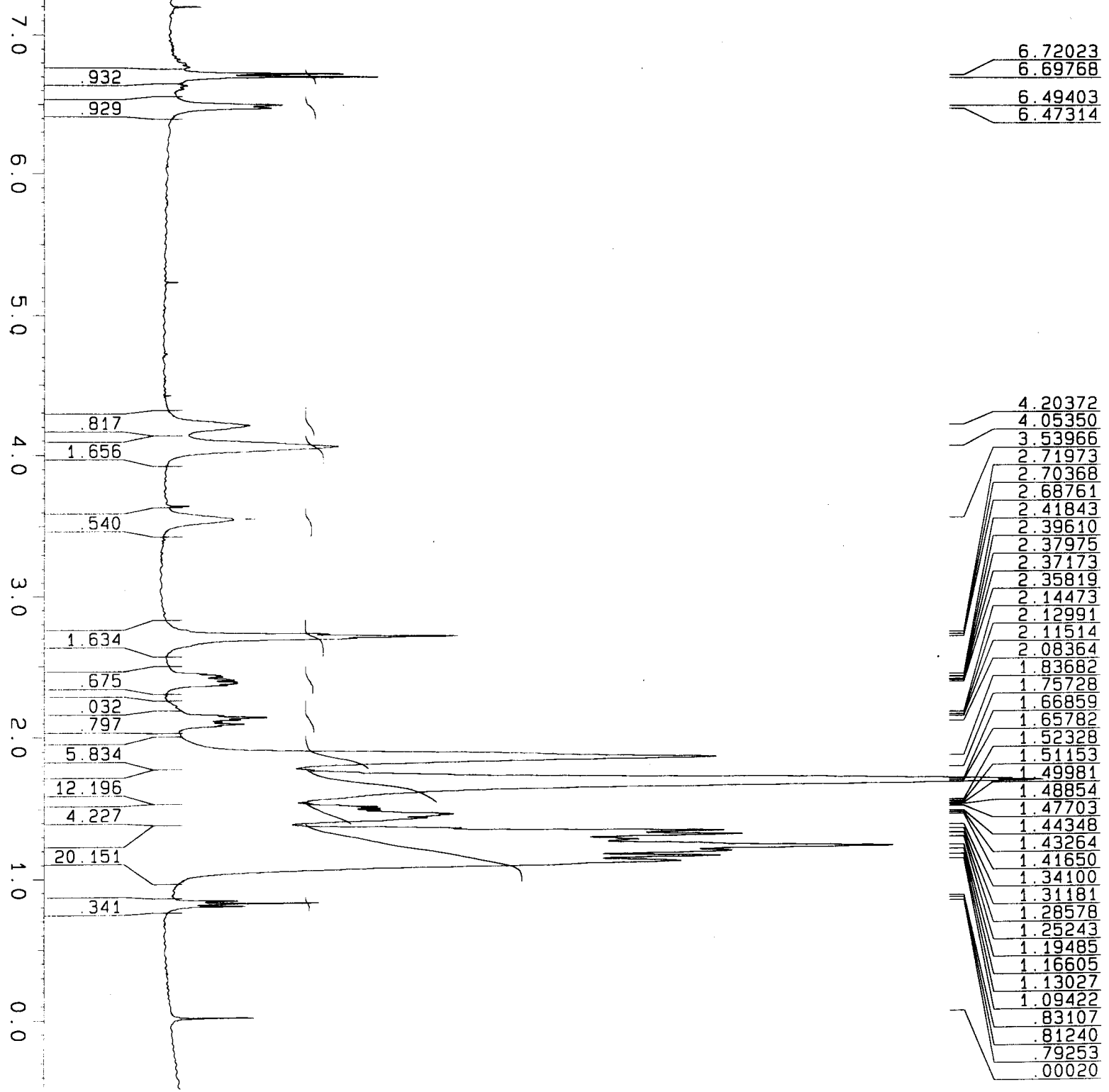

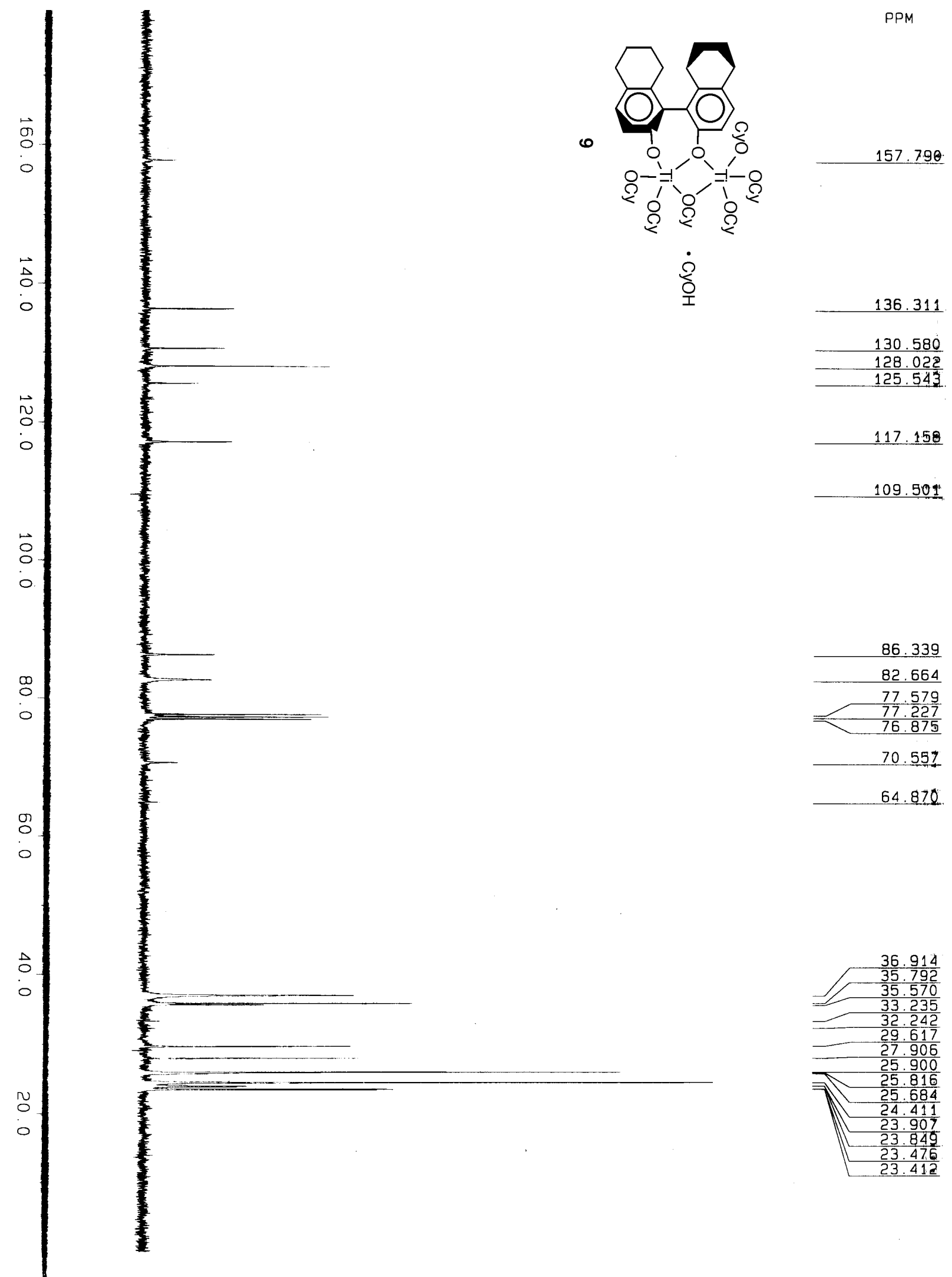


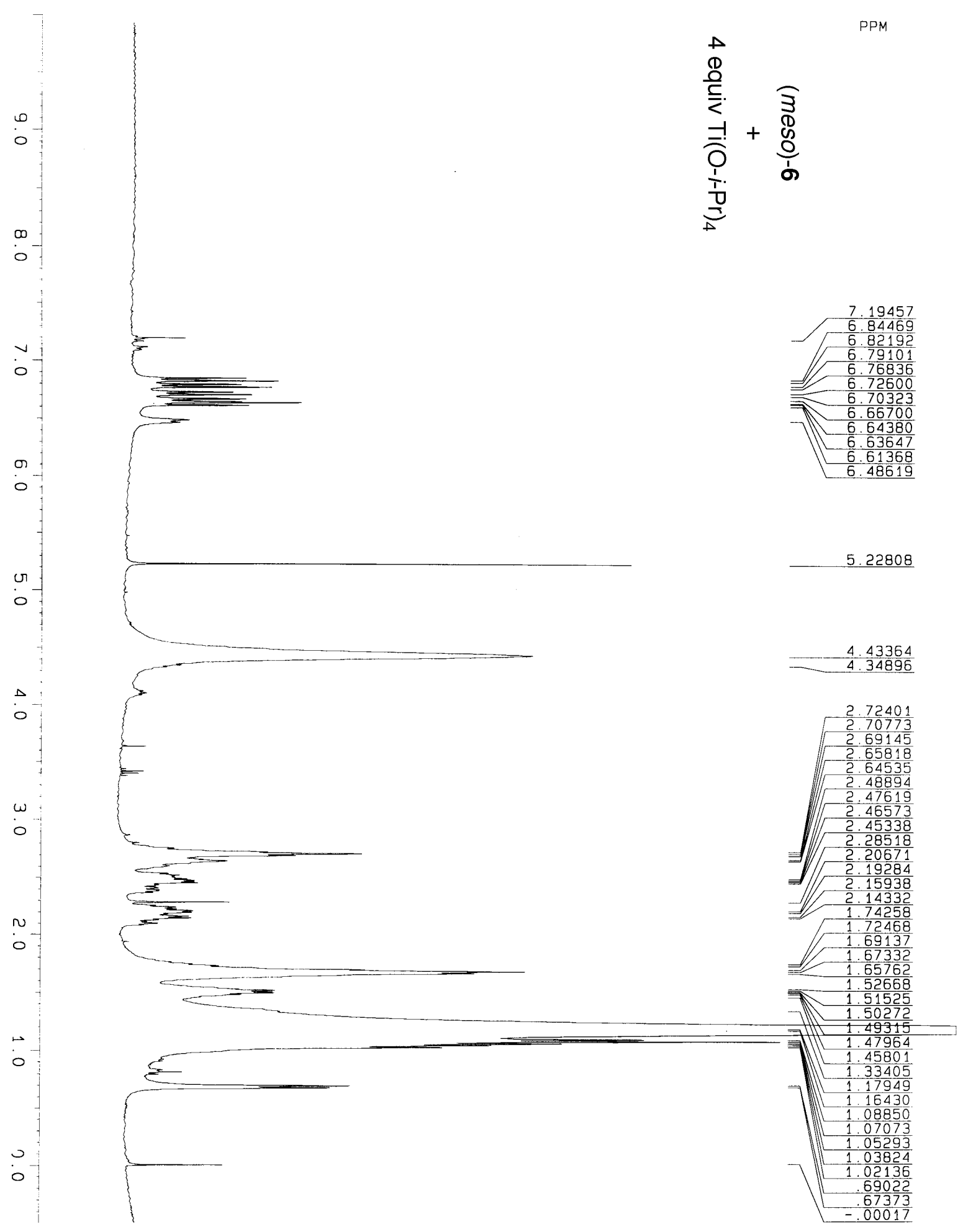




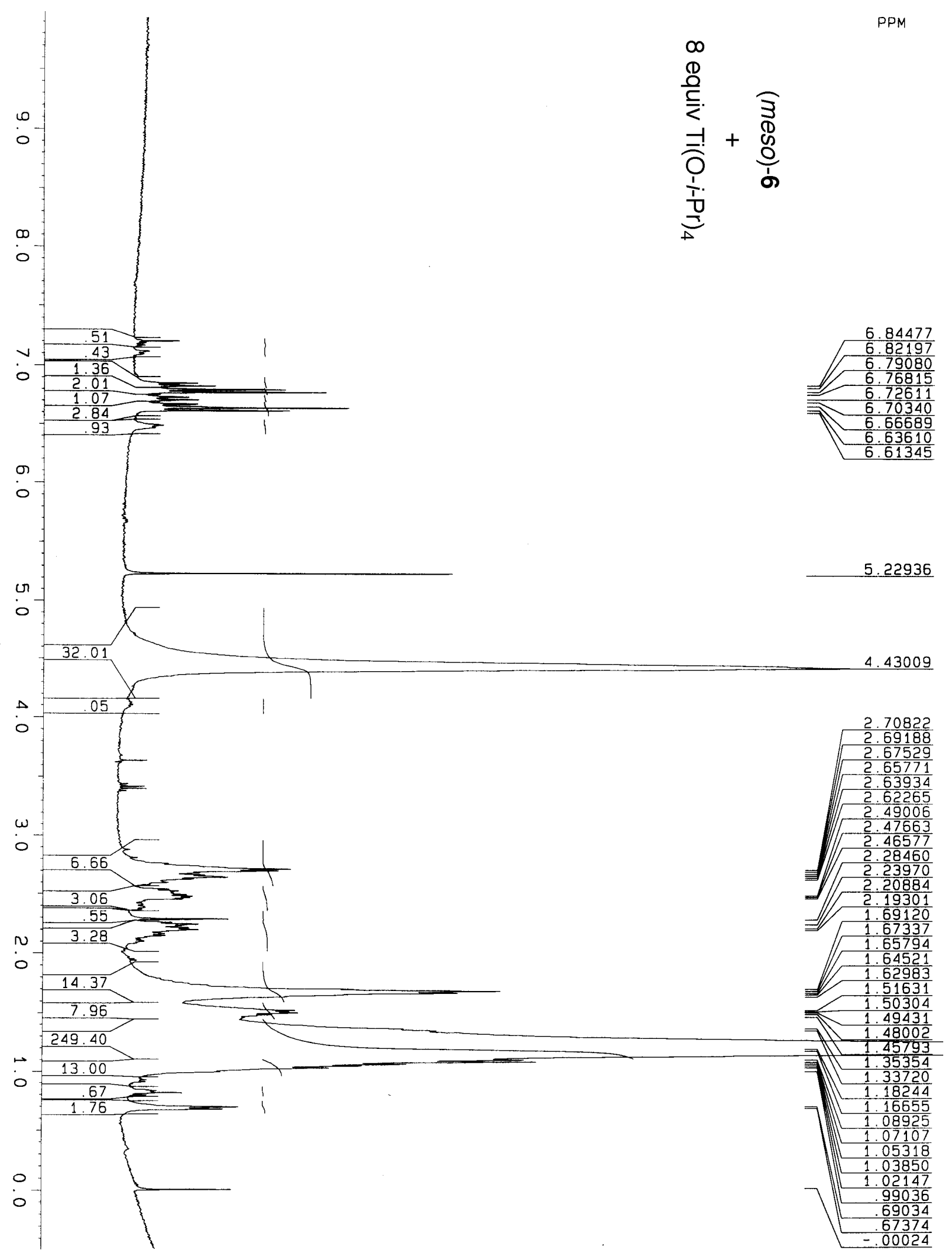




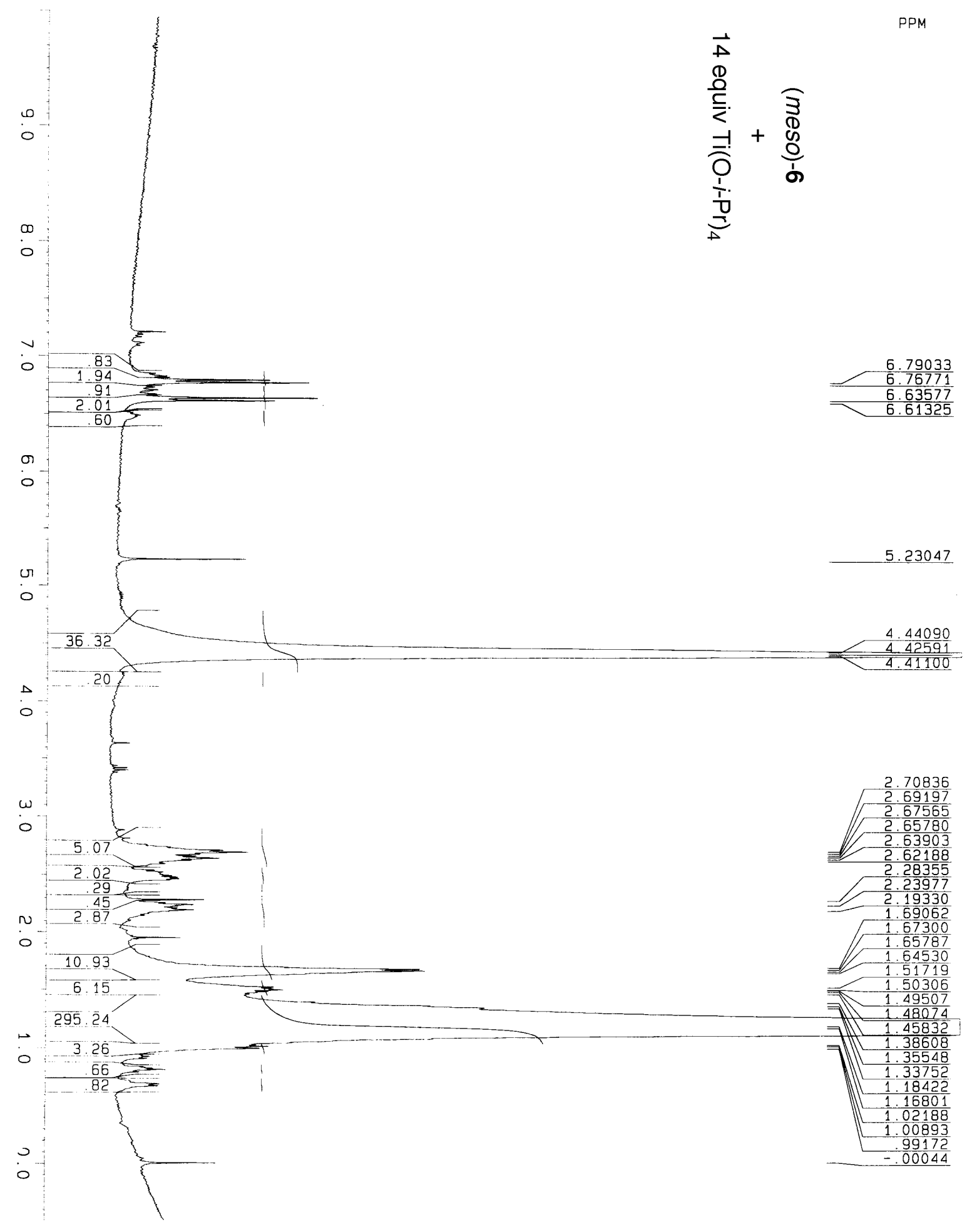




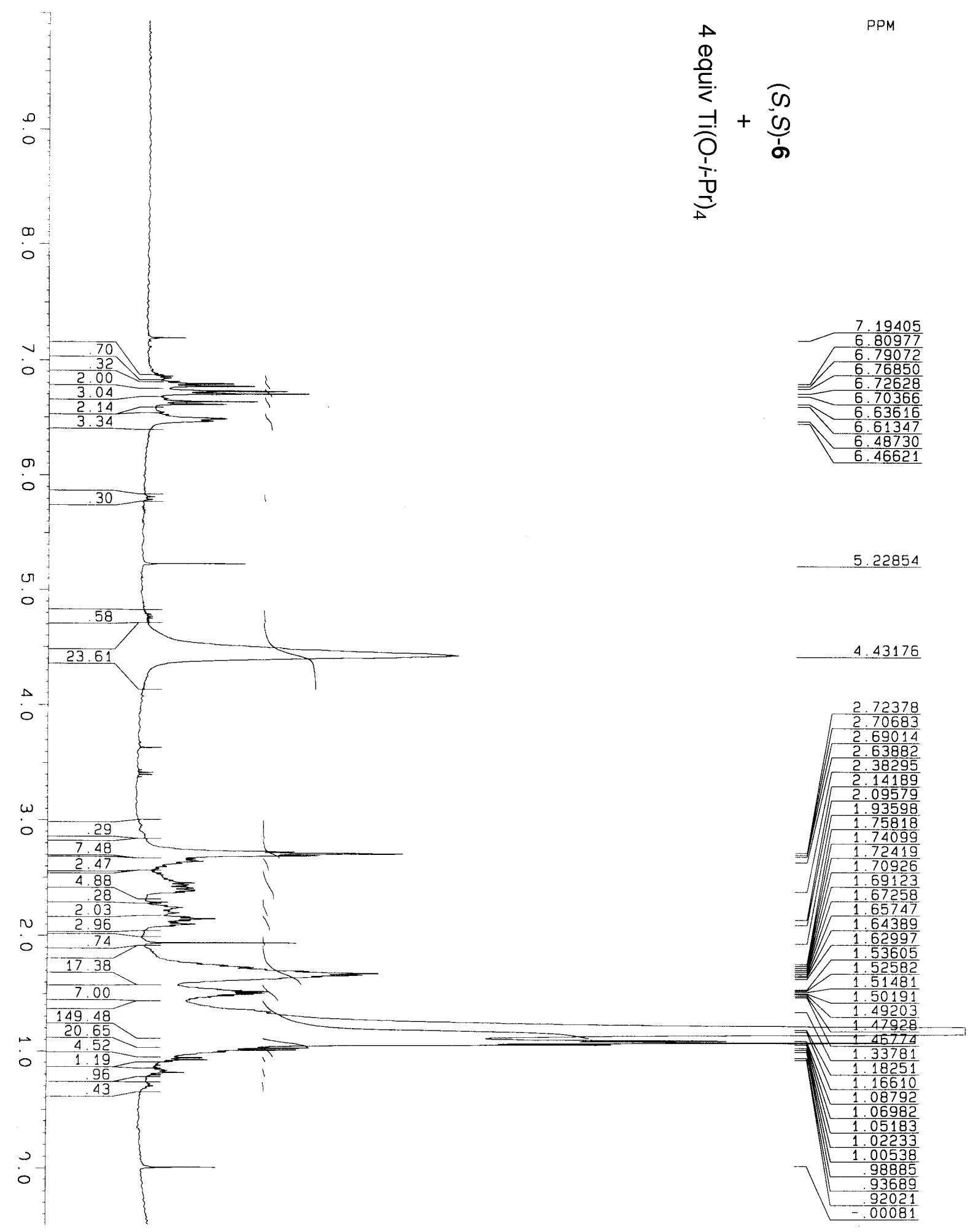




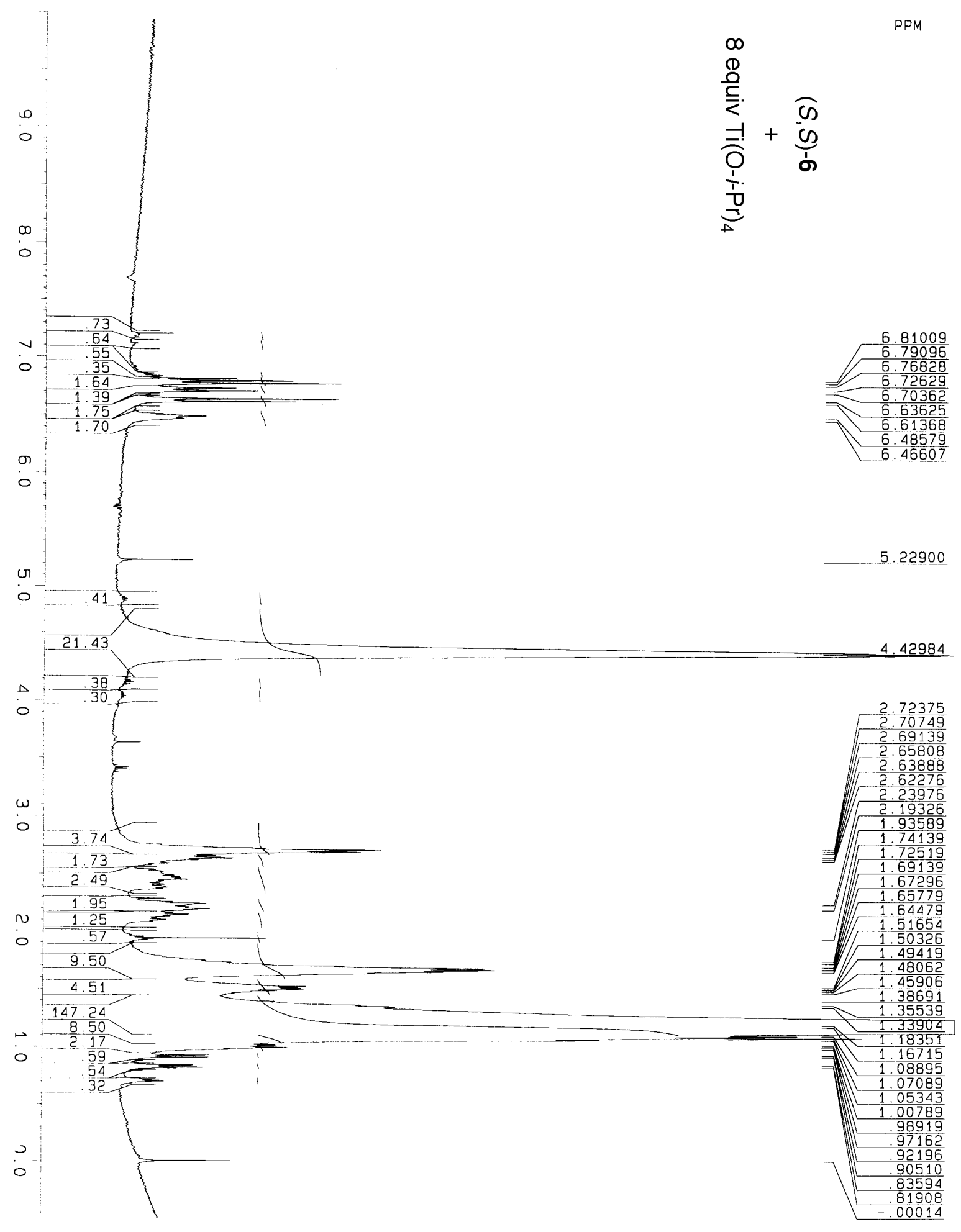




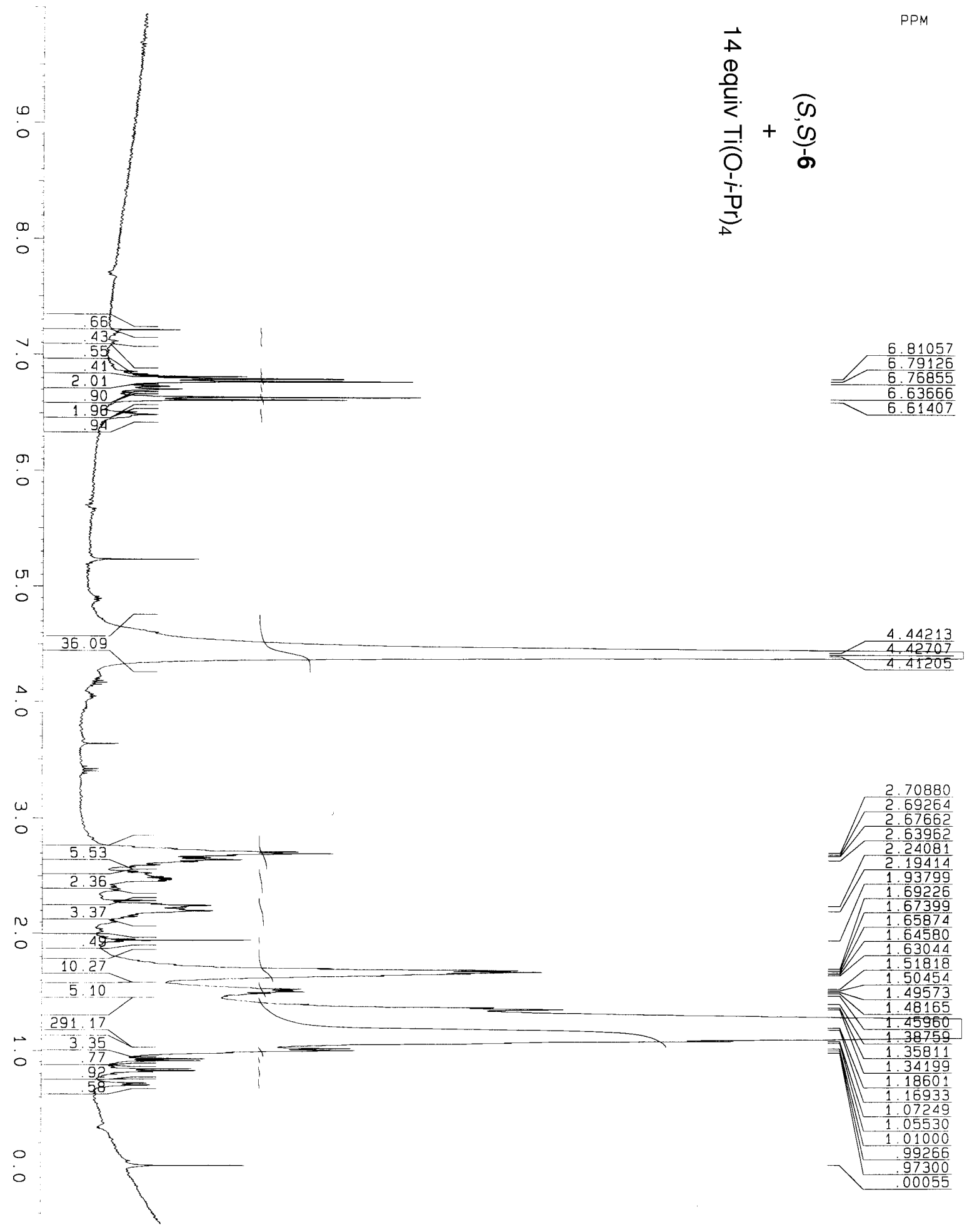



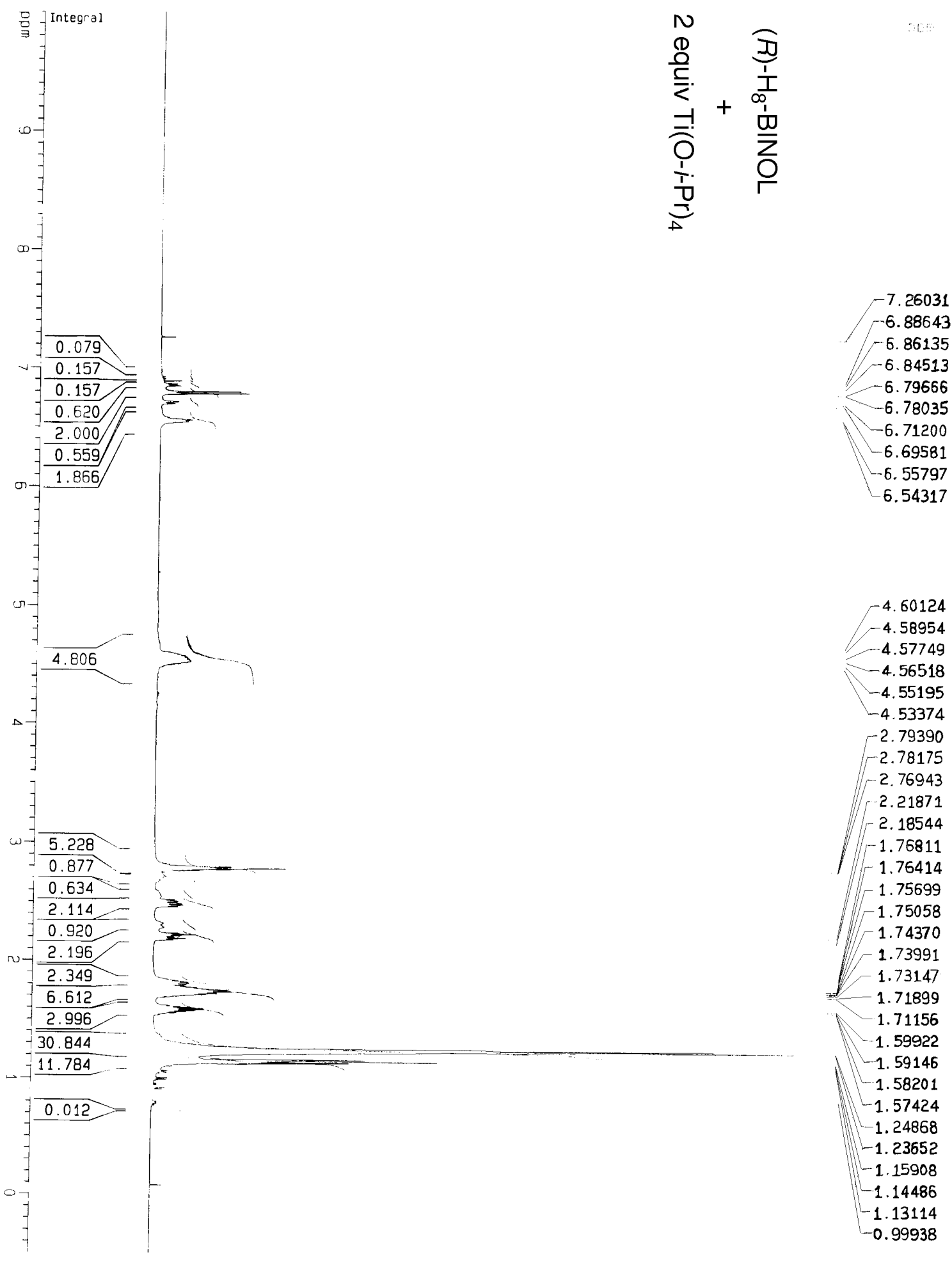

4.60124

$-4.58954$

$-4.57749$

$-4.56518$

$-4.55195$

$-4.53374$

$-2.79390$

$-2.78175$

$-2.76943$

2.21871

$-2.18544$

$-1.76811$

1.76414

$-1.75699$

$-1.75058$

$\sqrt{-1.74370}$

$/ /-1.73991$

- 1.73147

$-1.71899$

1.71156

-1. 59922

$-1.59146$

$-1.58201$

$-1.57424$

$-1.24868$

$-1.23652$

$-1.15908$

$-1.14486$

$-1.13114$

$-0.99938$ 


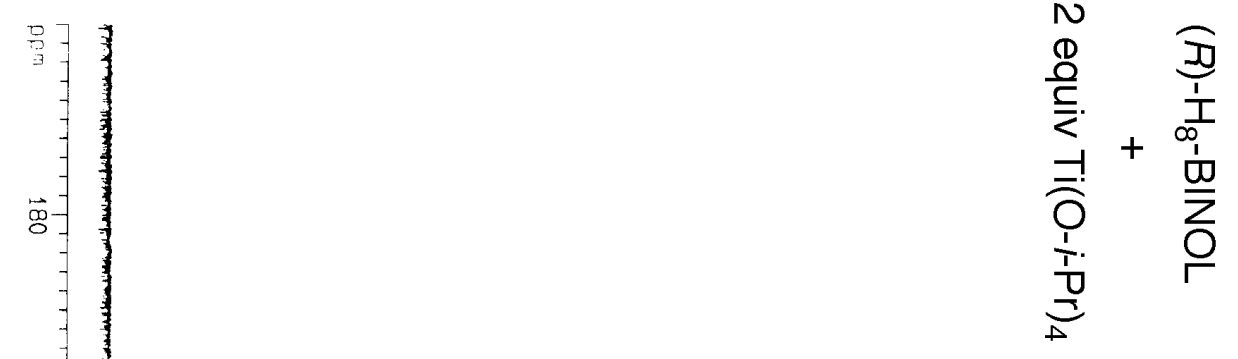

$-157.853$

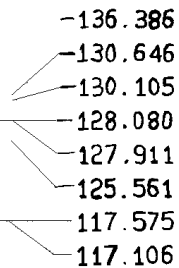

0

D-

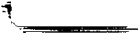

5

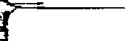

117. 106

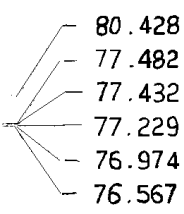

g-

$\circ-$
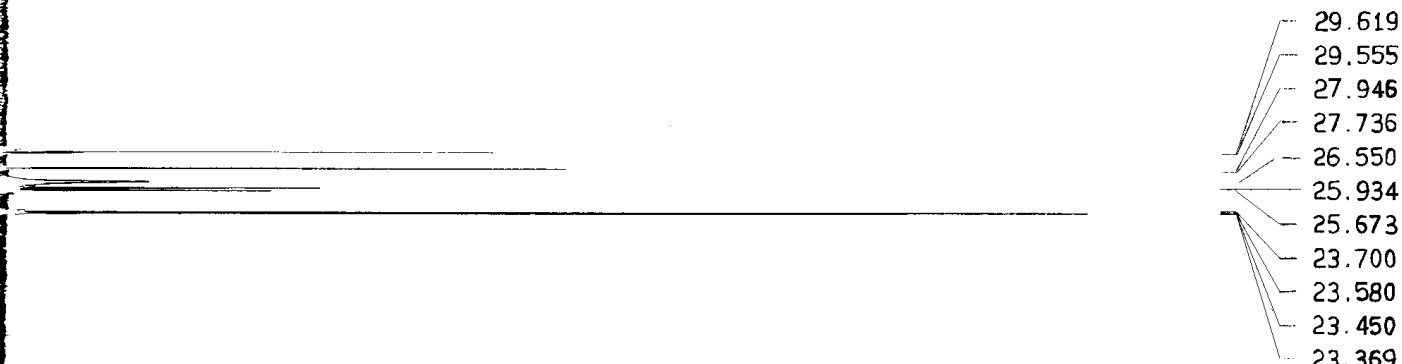

29.619

29.555

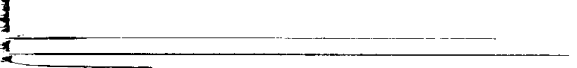

-1
-1
-1
-1
-1
-1
-1
-1

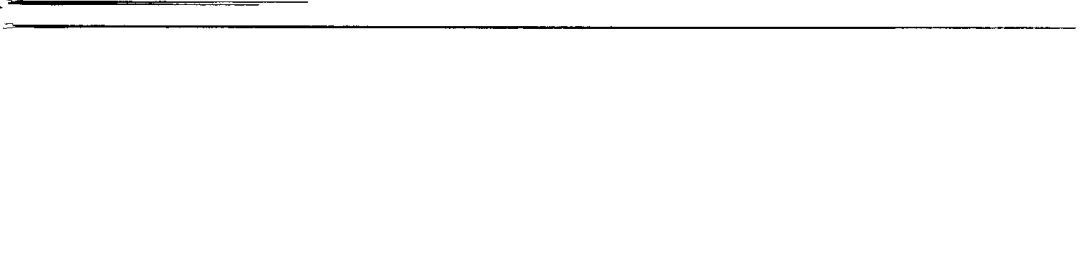

23. 369 


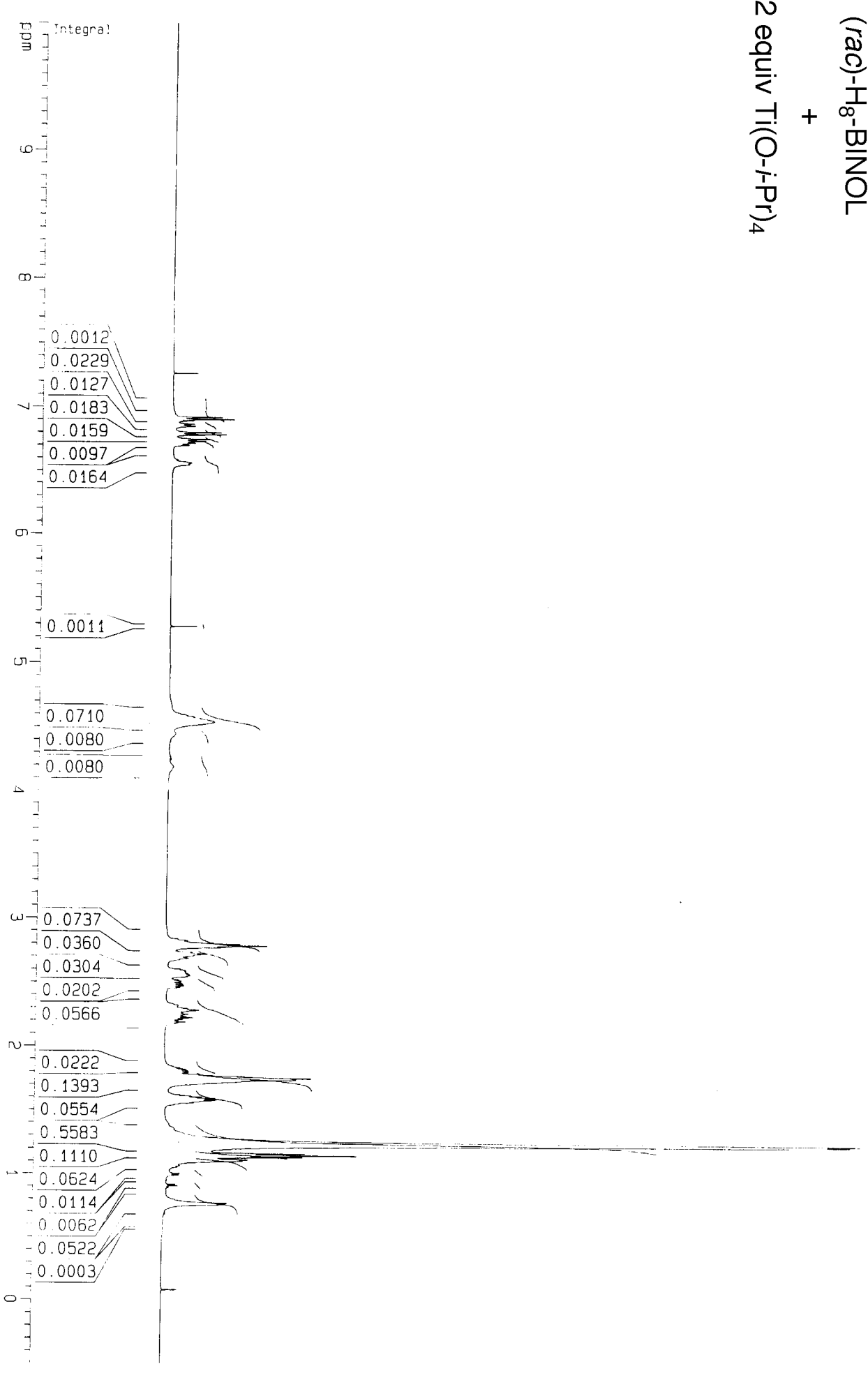

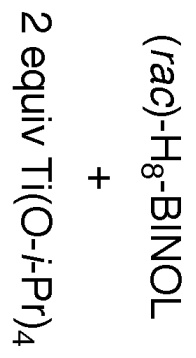
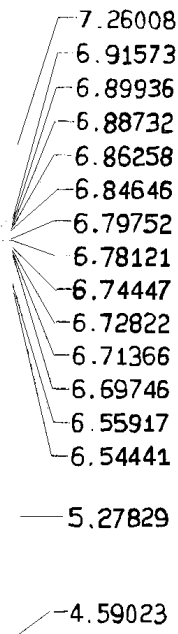

$-4.57811$

$-4.56517$

$-4.53728$

$-2.79330$

$-2.78214$

$-2.77026$

$-2.71857$

$-2.70664$

$-2.28100$

$-1.77536$

$-1.76221$

$-1.74995$

T -1.74304

- 1.73786

$-1.60003$

$-1.59168$

$-1.58279$

$-1.57497$

$-1.56609$

$-1.23813$

$-1.22889$

$-1.15973$

$-1.14569$

$-1.13192$

$-1.11109$

$-1.10081$

$-0.76715$

$-0.75692$ 


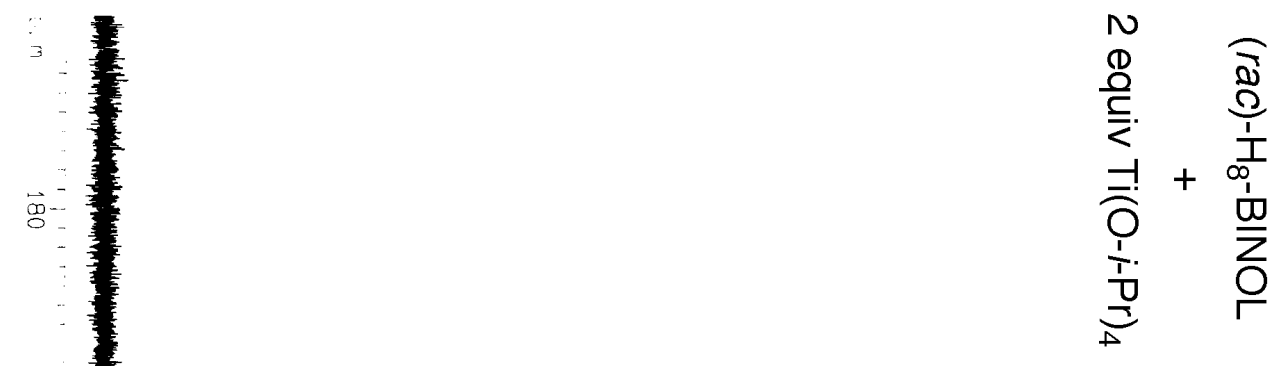

$-158.989$

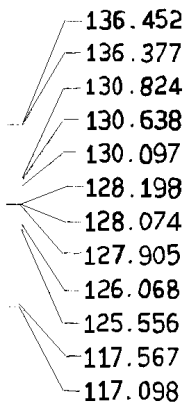

$\overrightarrow{8}$

9

吕-

$\overrightarrow{0}$

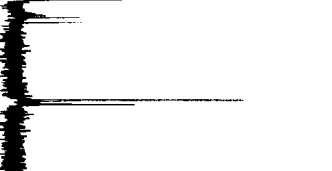

80.930

80.417

79.615

77.482

$-77.432$

77.229
-76.974

76.974

g.

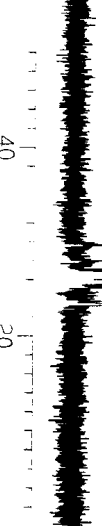

$-29.610$

$-29.576$

- 27.953

- 27.939

$-26.512$

$-25.925$

-25.757
-25.664

$-25.664$

- 25.351

$-25.213$

- 23.442

$-23.363$

-23.305
-23.363 


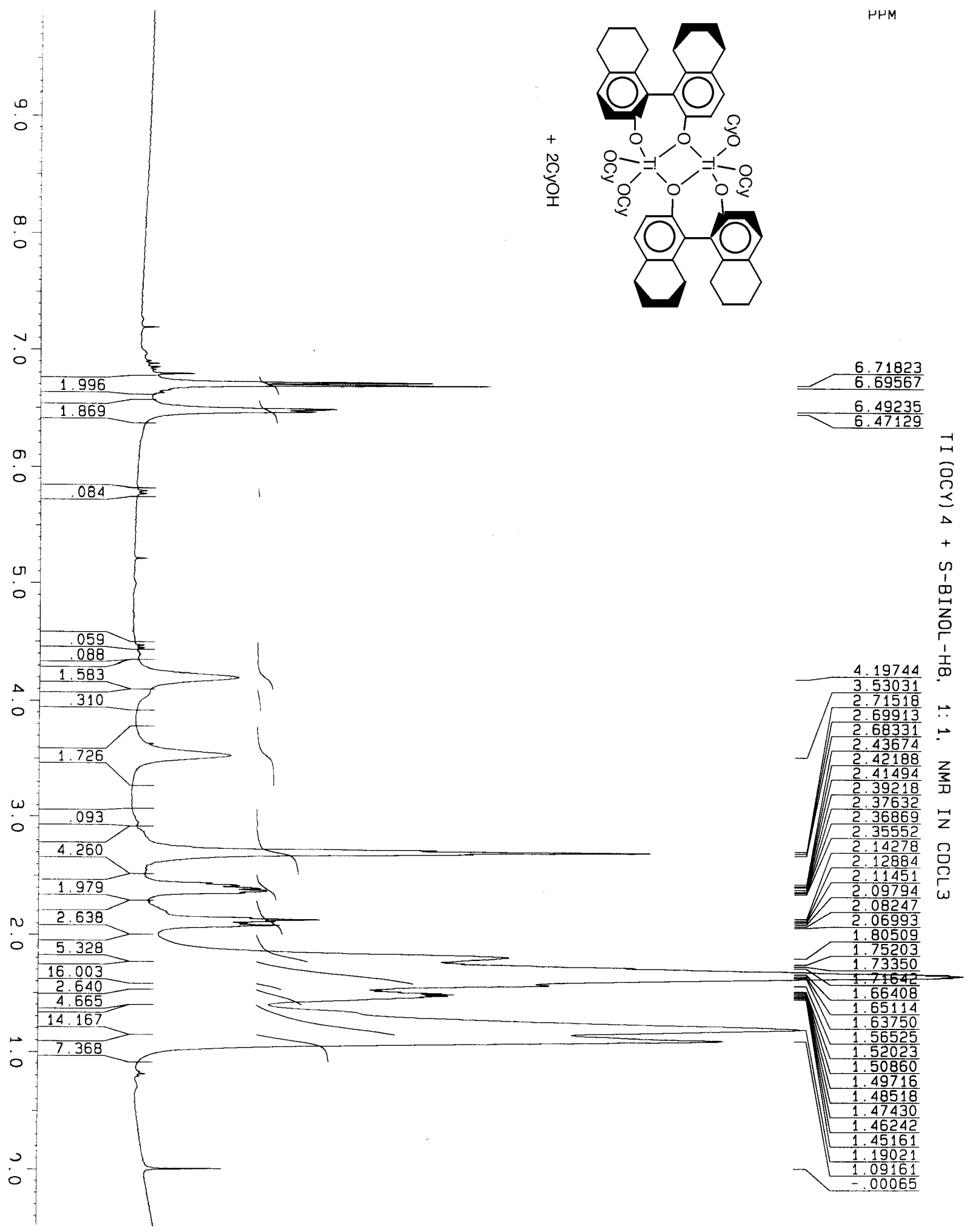



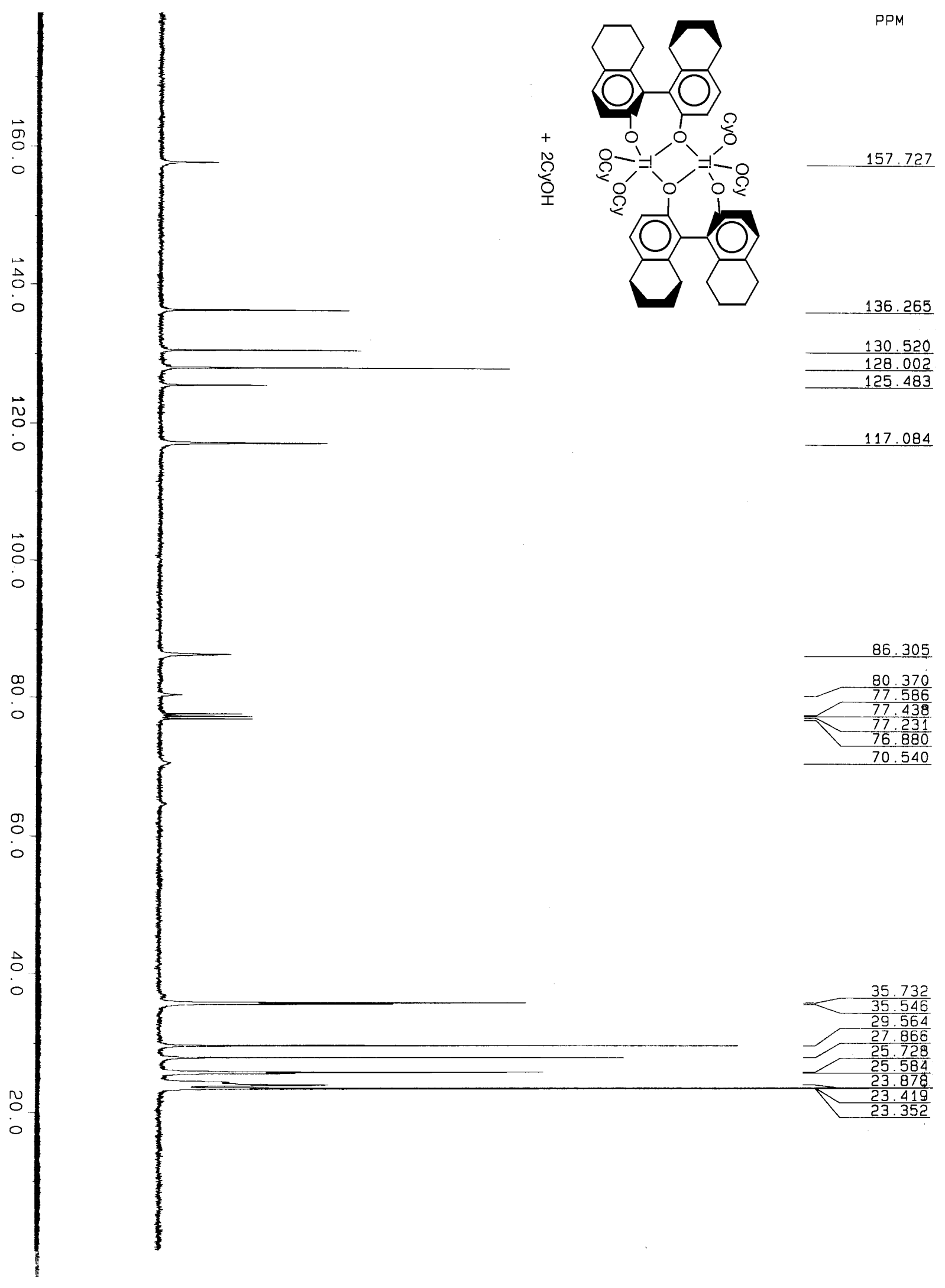

S52 